doi:10.1017/S1474748021000360 (C) The Author(s), 2021. Published by Cambridge University Press. This is an Open Access article, distributed under the terms of the Creative Commons Attribution licence (https://creativecommons.org/licenses/by/4.0), which permits unrestricted re-use, distribution and reproduction, provided the original article is properly cited.

\title{
TRANSITION DENSITIES OF SUBORDINATORS OF POSITIVE ORDER
}

\author{
TOMASZ GRZYWNY (D) ${ }^{1}$, ŁUKASZ LEŻAJ (D) ${ }^{1}$ AND BARTOSZ TROJAN (D) ${ }^{2}$ \\ ${ }^{1}$ Faculty of Pure and Applied Mathematics, Wroctaw University of Science and \\ Technology, wyb. Wyspiańskiego 27, 50-370 Wroctaw, Poland \\ (tomasz.grzywny@pwr.edu.pl; lukasz.lezaj@pwr.edu.pl) \\ ${ }^{2}$ Institute of Mathematics, Polish Academy of Sciences, ul. Śniadeckich 8, 00-656 \\ Warszawa, Poland \\ (btrojan@impan.pl)
}

(Received 29 March 2021; accepted 1 July 2021; first published online 22 October 2021)

\begin{abstract}
We prove the existence and asymptotic behaviour of the transition density for a large class of subordinators whose Laplace exponents satisfy lower scaling condition at infinity. Furthermore, we present lower and upper bounds for the density. Sharp estimates are provided if an additional upper scaling condition on the Laplace exponent is imposed. In particular, we cover the case when the (minus) second derivative of the Laplace exponent is a function regularly varying at infinity with regularity index bigger than -2 .
\end{abstract}

Keywords: subordination, heat kernel, Green function

2020 Mathematics subject classification: 60J35, 60J75, 47D03

\section{Introduction}

Asymptotic behaviour as well as estimates of heat kernels have been intensively studied in the last decades. The first results obtained by Pòlya [44] and Blumenthal and Getoor [4] for isotropic $\alpha$-stable process in $\mathbb{R}^{d}$ provided the basis for studies of more complicated processes; for example, subordinated Brownian motions [40, 49], isotropic unimodal Lévy processes [6, 15, 21] and even more general symmetric Markov processes [11, 14]. One may, among others, list the articles on heat kernel estimates for jump processes of finite range [10] or with lower intensity of higher jumps [39, 51]. While a great many articles with explicit results are devoted to symmetric processes or those which are, in appropriate sense, similar to the symmetric ones, the nonsymmetric case is in general harder to handle due to lack of a familiar structure. This problem was approached in many different ways; see, for example, [7, 27, 28, 31, 32, 35, 36, 42, 43, 50]. For a more specific class of stable processes, see $[25,46,53]$. Overall, one has to impose some control on the nonsymmetry in order to obtain estimates in an easy-to-handle form. This idea was applied in the recent 
paper [22] where the authors considered the case of the Lévy measure being comparable to some unimodal Lévy measure. The methods developed in [22, 23] contributed significantly to this article. See also [32, 42] and the references therein.

In this article the central object is a subordinator; that is, a one-dimensional Lévy process with nondecreasing paths starting at 0 ; see Section 2 for the precise definition. The abstract introduction of the subordination dates back to 1950s and is due to Bochner [5] and Philips [41]. In the language of the semigroup theory, for a Bernstein function $\phi$ and a bounded $C_{0}$-semigroup $\left(e^{-t \mathcal{A}}: t \geq 0\right)$ with $-\mathcal{A}$ being its generator on some Banach space $\mathscr{X}$, via Bochner integral one can define an operator $\mathcal{B}=\phi(\mathcal{A})$ such that $-\mathcal{B}$ also generates a bounded $C_{0}$-semigroup $\left(e^{-t \mathcal{B}}: t \geq 0\right)$ on $\mathscr{X}$. The semigroup $\left(e^{-t \mathcal{B}}: t \geq 0\right)$ is then said to be subordinated to $\left(e^{-t \mathcal{A}}: t \geq 0\right)$, and although it may be very different from the original one, its properties clearly follow from properties of both the parent semigroup and the involved Bernstein function. See, for example, [18] and the references therein. From a probabilistic point of view, due to positivity and monotonicity, subordinators naturally appear as random time change functions of Lévy processes or, more generally, Markov processes. Namely, if $\left(X_{t}: t \geq 0\right)$ is a Markov process and $\left(T_{t}: t \geq 0\right)$ is an independent subordinator, then $Y_{t}=X_{T_{t}}$ is again a Markov process with a transition function given by

$$
\mathbb{P}^{x}\left(Y_{t} \in A\right)=\int_{[0, \infty)} \mathbb{P}^{x}\left(X_{s} \in A\right) \mathbb{P}\left(T_{t} \in \mathrm{d} s\right) .
$$

The procedure just described is called a subordination of a Markov process and can be interpreted as a probabilistic form of the equality $\mathcal{B}=\phi(\mathcal{A})$. Here $\mathcal{A}$ and $\mathcal{B}$ are (minus) generators of semigroups associated to processes $X_{t}$ and $Y_{t}$, respectively. From an analytical point of view, the transition density of $Y_{t}$ (the integral kernel of $e^{-t \mathcal{B}}$ ) can be obtained as a time average of transition density of $X_{t}$ with respect to distribution of $T_{t}$. Yet another approach is driven by partial differential equations, as the transition density is a heat kernel of a generalised heat equation. The generalisation can be twofold: either by replacing the Laplace operator with another, possibly nonlocal operator or by introducing a more general fractional-time derivative instead of the classical one. The latter case was recently considered in $[9,12,38]$. Here the solutions are expressed in terms of corresponding (inverse) subordinators and thus their analysis is essential.

By taking $\mathcal{A}=-\Delta$ and changing the time of (i.e., subordinating) Brownian motion one can obtain a large class of subordinated Brownian motions. A principal example here is an $\alpha$-stable subordinator with the Laplace exponent $\phi(\lambda)=\lambda^{\alpha}, \alpha \in(0,1)$, which gives rise to the symmetric, rotation-invariant $\alpha$-stable process and corresponds to the special case of fractional powers of semigroup $\left(e^{-t \mathcal{A}^{\alpha}}: t \geq 0\right)$. For this reason, distributional properties of subordinators were often studied with reference to heat kernel estimates of subordinated Brownian motions (see, e.g., [16, 33]). In [24] Hawkes investigated the growth of sample paths of a stable subordinator and obtained the asymptotic behaviour of its distribution function. Jain and Pruitt [30] considered tail probability estimates for subordinators and, in the discrete case, nondecreasing random walks. In a more general setting some related results were obtained in $[17,26,42,52]$. In [8] new examples of families of subordinators with explicit transition densities were given. Finally, in the recent paper [16], the author 
derived explicit approximate expressions for the transition density of approximately stable subordinators under very restrictive assumptions.

The result of the article is asymptotic behaviour as well as upper and lower estimates of transition densities of subordinators satisfying scaling condition imposed on the second derivative of the Laplace exponent $\phi$. Our standing assumption on $-\phi^{\prime \prime}$ is the weak lower scaling condition at infinity with scaling parameter $\alpha-2$, for some $\alpha>0$ (see (2.7) for definition). It is worth highlighting that we do not state our assumptions and results in terms of the Laplace exponent $\phi$, as one could suspect, but in terms of its second derivative and related function $\varphi(x)=x^{2}\left(-\phi^{\prime \prime}(x)\right.$ ) (see Theorems 3.3, 4.7 and 4.8). Usually the transition density of a Lévy process is described by the generalised inverse of the real part of the characteristic exponent $\psi^{-1}(x)$ (e.g., [23], [36]), but in our setting one can show that the lower scaling property implies that $\varphi^{-1}(x) \approx \psi^{-1}(x)$ for $x$ sufficient large (see Proposition 4.3). In some cases, however, $\varphi$ may be significantly different from the Laplace exponent $\phi$. However, if one assumes additional upper scaling condition with scaling parameter $\beta-2$ for $\beta$ strictly between 0 and 1 , then these two objects are comparable (see Proposition 4.6).

The main results of this article are covered by Theorems 3.3, 4.7, 4.8, 4.11 and 4.17. Theorem 3.3 is essential for the whole article because it provides not only the existence of the transition density but also its asymptotic behaviour, which is later used in derivation of upper and lower estimates. The key argument in the proof is the lower estimate on the holomorphic extension of the Laplace exponent $\phi$ (see Lemma 3.1), which justifies the inversion of the Laplace transform and allows us to perform the saddle point type approximation. In Theorem 3.3 we only use the weak lower scaling property on the second derivative of the Laplace exponent. In particular, we do not assume the absolute continuity of $\nu(\mathrm{d} x)$. Furthermore, the asymptotic is valid in some region described in terms of both space and time variables. By freezing one of them, we obtain as corollaries the results similar to [16]; see, for example, Corollary 3.6. It is also worth highlighting that we obtain a version of the upper estimate on the transition density with no additional assumptions on the Lévy measure $\nu(\mathrm{d} x)$; see Theorem 4.7. Clearly, putting some restrictions on $\nu(\mathrm{d} x)$ results in sharper estimates (Theorem 4.8), but it is interesting that the scaling property alone is enough to get some information. Our starting point and the main object to work with is the Laplace exponent $\phi$. However, in many cases the primary object is the Lévy measure $\nu(\mathrm{d} x)$ and results are presented in terms of or require its tail decay. Therefore, it would be convenient to have a connection between those two objects. In Proposition 3.8 we prove that one can impose scaling conditions on the tail of the Lévy measure $\nu((x, \infty))$ instead, as they imply the scaling condition on $-\phi^{\prime \prime}$.

We also note that the main results of the article hold true when $-\phi^{\prime \prime}$ is a function regularly varying at infinity with regularity index $\alpha-2$, where $\alpha>0$. This follows easily by Potter bounds for regularly varying functions (see [3, Theorem 1.5.6]), which immediately imply both lower and upper scaling properties. Moreover, if additionally $\alpha<1$, then, by Karamata's theorem and monotone density theorem, regular variation of $-\phi^{\prime \prime}$ with index $\alpha-2$ is equivalent to regular variation of $\phi$ with index $\alpha$. This is not the case for the case $\alpha=1$ where, in general, only the first direction holds true. 
Below we present the special case when global upper and lower scaling conditions are imposed with $0<\alpha \leq \beta<1$; see Theorem 4.17.

Theorem A. Let $\mathbf{T}$ be a subordinator with the Laplace exponent $\phi$. Suppose that for some $0<\alpha \leq \beta<1$, the functions

$$
(0, \infty) \ni x \mapsto x^{-\alpha} \phi(x), \quad \text { and } \quad(0, \infty) \ni x \mapsto x^{-\beta} \phi(x)
$$

are almost increasing and almost decreasing, respectively. We also assume that the Lévy measure $\nu(\mathrm{d} x)$ has an almost monotone density $\nu(x)$. Then the probability distribution of $T_{t}$ has a density $p(t, \cdot)$. Moreover, for all $t \in(0, \infty)$ and $x>0$,

$$
p(t, x) \approx \begin{cases}\left(t\left(-\phi^{\prime \prime}(w)\right)\right)^{-1 / 2} \exp \left\{-t\left(\phi(w)-w \phi^{\prime}(w)\right)\right\}, & \text { if } 0<x \phi^{-1}(1 / t) \leq 1, \\ t x^{-1} \phi(1 / x), & \text { if } 1<x \phi^{-1}(1 / t),\end{cases}
$$

where $w=\left(\phi^{\prime}\right)^{-1}(x / t)$.

We note that a similar result to Theorem A appeared in [13] in around the same time as our preprint. Our assumptions, however, are weaker, as we assume almost monotonicity of the Lévy density instead of monotonicity of the function $t \mapsto t \nu(t)$. Moreover, our estimates are genuinely sharp; that is, the constants appearing in the exponential factors are the same on both sides of the estimate, while estimates obtained in [13] are qualitatively sharp; that is, the constants in the exponential factors are different.

As a corollary, under the assumption of Theorem A, we obtain a global two-sided estimate on the Green function. Namely, for all $x>0$,

$$
G(x) \approx \frac{1}{x \phi(1 / x)}
$$

See Section 5 and Theorem 5.8 for details.

The article is organised as follows: In Section 2 we introduce our framework and collect some facts concerning Bernstein functions and their scaling properties. Section 3 is devoted to the proof of Theorem 3.3 and its consequences. In Section 4 we provide both upper and lower estimates on the transition density and discuss when these estimates coincide. Some applications of our results to subordination beyond the familiar $\mathbb{R}^{d}$ setting and Green function estimates are presented in Section 5.

\section{Notation}

By $C_{1}, c_{1}, C_{2}, c_{2}, \ldots$ we denote positive constants which may change from line to line. For two functions $f, g:(0, \infty) \rightarrow[0, \infty)$ we write $f(x) \gtrsim g(x)$ if there is $c>0$ such that $f(x) \geq c g(x)$ for all $x>0$. An analogous rule is applied to the symbol $\lesssim$. We also have $f(x) \approx g(x)$ if there exists $C \geq 1$ such that $C^{-1} f(x) \leq g(x) \leq C f(x)$ for all $x>0$. Finally, we set $a \wedge b=\min \{a, b\}$ and $a \vee b=\max \{a, b\}$.

\section{Preliminaries}

Let $(\Omega, \mathcal{F}, \mathbb{P})$ be a probability space. Let $\mathbf{T}=\left(T_{t}: t \geq 0\right)$ be a subordinator; that is, a Lévy process in $\mathbb{R}$ with nondecreasing paths. Recall that a Lévy process is a càdlàg stochastic 
process with stationary and independent increments such that $T_{0}=0$ almost surely. There is a function $\psi: \mathbb{R} \rightarrow \mathbb{C}$, called the Lévy-Khintchine exponent of $\mathbf{T}$, such that for all $t \geq 0$ and $\xi \in \mathbb{R}$,

$$
\mathbb{E}\left(e^{i \xi T_{t}}\right)=e^{-t \psi(\xi)}
$$

Moreover, there are $b \geq 0$ and $\sigma$-finite measure $\nu$ on $(0, \infty)$ satisfying

$$
\int_{(0, \infty)} \min \{1, s\} \nu(\mathrm{d} s)<\infty
$$

such that for all $\xi \in \mathbb{R}$,

$$
\begin{aligned}
\psi(\xi) & =-i \xi b-\int_{(0, \infty)}\left(e^{i \xi x}-1\right) \nu(\mathrm{d} x) \\
& =-i \xi\left(b+\int_{(0,1)} x \nu(\mathrm{d} x)\right)-\int_{(0, \infty)}\left(e^{i \xi x}-1-i \xi x \mathbb{1}_{\{x<1\}}\right) \nu(\mathrm{d} x)
\end{aligned}
$$

which is valid thanks to $(2.1)$. By $\phi:[0, \infty) \rightarrow[0, \infty)$ we denote the Laplace exponent of $\mathbf{T}$, namely,

$$
\mathbb{E}\left(e^{-\lambda T_{t}}\right)=e^{-t \phi(\lambda)}
$$

for all $t \geq 0$ and $\lambda \geq 0$. Let $\psi^{*}$ be the symmetric continuous and nondecreasing majorant of $\Re \psi$; that is,

$$
\psi^{*}(r)=\sup _{|z| \leq r} \Re \psi(z), \quad r>0 .
$$

Notice that

$$
\psi^{*}\left(\psi^{-1}(s)\right)=s, \quad \text { and } \quad \psi^{-1}\left(\psi^{*}(s)\right) \geq s,
$$

where $\psi^{-1}$ is the generalised inverse function defined as

$$
\psi^{-1}(s)=\sup \left\{r>0: \psi^{*}(r)=s\right\} .
$$

To study the distribution function of the subordinator $\mathbf{T}$, it is convenient to introduce two concentration functions $K$ and $h$. They are defined as

$$
K(r)=\frac{1}{r^{2}} \int_{(0, r)} s^{2} \nu(\mathrm{d} s), \quad r>0
$$

and

$$
h(r)=\int_{(0, \infty)} \min \left\{1, r^{-2} s^{2}\right\} \nu(\mathrm{d} s), \quad r>0 .
$$

Notice that $h(r) \geq K(r)$. Moreover, by the Fubini-Tonelli theorem, we get

$$
h(r)=2 \int_{r}^{\infty} K(s) s^{-1} \mathrm{~d} s .
$$


In view of [20, Lemma 4], we have

$$
\frac{1}{24} h\left(r^{-1}\right) \leq \psi^{*}(r) \leq 2 h\left(r^{-1}\right) .
$$

A function $f:[0, \infty) \rightarrow[0, \infty)$ is regularly varying at infinity of index $\alpha$, if for all $\lambda \geq 1$,

$$
\lim _{x \rightarrow \infty} \frac{f(\lambda x)}{f(x)}=\lambda^{\alpha} .
$$

Analogously, $f$ is regular varying at the origin of index $\alpha$ if for all $\lambda \geq 1$,

$$
\lim _{x \rightarrow 0^{+}} \frac{f(\lambda x)}{f(x)}=\lambda^{\alpha} .
$$

If $\alpha=0$, the function $f$ is called slowly varying.

We next introduce a notion of scaling conditions frequently used in this article. We say that a function $f:[0, \infty) \rightarrow[0, \infty)$ has the weak lower scaling property at infinity if there are $\alpha \in \mathbb{R}, c \in(0,1]$ and $x_{0} \geq 0$ such that for all $\lambda \geq 1$ and $x>x_{0}$,

$$
f(\lambda x) \geq c \lambda^{\alpha} f(x) .
$$

We denote it briefly as $f \in \operatorname{WLSC}\left(\alpha, c, x_{0}\right)$. Observe that if $\alpha>\alpha^{\prime}$ then $\operatorname{WLSC}\left(\alpha, c, x_{0}\right) \subsetneq$ WLSC $\left(\alpha^{\prime}, c, x_{0}\right)$. Analogously, $f$ has the weak upper scaling property at infinity if there are $\beta \in \mathbb{R}, C \geq 1$, and $x_{0} \geq 0$ such that for all $\lambda \geq 1$ and $x>x_{0}$,

$$
f(\lambda x) \leq C \lambda^{\beta} f(x) .
$$

In this case we write $f \in \operatorname{WUSC}\left(\beta, C, x_{0}\right)$.

We say that a function $f:[0, \infty) \rightarrow[0, \infty)$ has doubling property on $\left(x_{0}, \infty\right)$ for some $x_{0} \geq 0$ if there is $C \geq 1$ such that for all $x>x_{0}$,

$$
C^{-1} f(x) \leq f(2 x) \leq C f(x) .
$$

Notice that a nonincreasing function with the weak lower scaling has a doubling property. Analogously, a nondecreasing function with the weak upper scaling.

A function $f:[0, \infty) \rightarrow[0, \infty)$ is almost increasing on $\left(x_{0}, \infty\right)$ for some $x_{0} \geq 0$ if there is $c \in(0,1]$ such that for all $y \geq x>x_{0}$,

$$
c f(x) \leq f(y) .
$$

It is almost decreasing on $\left(x_{0}, \infty\right)$ if there is $C \geq 1$ such that for all $y \geq x>x_{0}$,

$$
C f(x) \geq f(y) \text {. }
$$

In view of [6, Lemma 11], $f \in \operatorname{WLSC}\left(\alpha, c, x_{0}\right)$ if and only if the function

$$
\left(x_{0}, \infty\right) \ni x \mapsto x^{-\alpha} f(x)
$$

is almost increasing. Similarly, $f \in \operatorname{WUSC}\left(\beta, C, x_{0}\right)$ if and only if the function

$$
\left(x_{0}, \infty\right) \ni x \mapsto x^{-\beta} f(x)
$$


is almost decreasing. For a function $f:[0, \infty) \rightarrow \mathbb{C}$ its Laplace transform is defined as

$$
\mathcal{L} f(\lambda)=\int_{0}^{\infty} e^{-\lambda x} f(x) \mathrm{d} x .
$$

\subsection{Bernstein functions}

In this section we recall some basic facts about Bernstein functions. A general reference here is the book [48].

A function $f:(0, \infty) \rightarrow[0, \infty)$ is completely monotone if it is smooth and

$$
(-1)^{n} f^{(n)} \geq 0
$$

for all $n \in \mathbb{N}_{0}$. Next, a function $\phi$ is a Bernstein function if it is a nonnegative smooth function such that $\phi^{\prime}$ is completely monotone.

Let $\phi$ be a Bernstein function. In view of [29, Lemma 3.9.34], for all $n \in \mathbb{N}$ we have

$$
\phi(\lambda) \geq \frac{(-1)^{n+1}}{n !} \lambda^{n} \phi^{(n)}(\lambda), \quad \lambda>0 .
$$

Since $\phi$ is concave, for each $\lambda \geq 1$ and $x>0$ we have

$$
\phi(\lambda x) \leq \phi^{\prime}(x)(\lambda-1) x+\phi(x) ;
$$

thus, by (2.9),

$$
\phi(\lambda x) \leq \lambda \phi(x)
$$

By [48, Theorem 3.2], there are two nonnegative numbers $a$ and $b$ and a Radon measure $\mu$ on $(0, \infty)$ satisfying

$$
\int_{(0, \infty)} \min \{1, s\} \mu(\mathrm{d} s)<\infty
$$

and such that

$$
\phi(\lambda)=a+b \lambda+\int_{(0, \infty)}\left(1-e^{-\lambda s}\right) \mu(\mathrm{d} s) .
$$

A Bernstein function $\phi$ is called a complete Bernstein function if the measure $\mu$ has a completely monotone density with respect to the Lebesgue measure.

Proposition 2.1. Let $f$ be a completely monotone function. Suppose that $f$ has a doubling property on $\left(x_{0}, \infty\right)$ for some $x_{0} \geq 0$. Then there is $C>0$ such that for all $x>x_{0}$,

$$
f(x) \geq C x\left|f^{\prime}(x)\right|
$$

Proof. Without loss of generality, we can assume $f \not \equiv 0$. Clearly,

$$
f(x)-f(x / 2)=\int_{x / 2}^{x} f^{\prime}(s) \mathrm{d} s \leq \frac{1}{2} x f^{\prime}(x) .
$$

Since $f$ is completely monotone, it is a positive function and

$$
f(x / 2) \geq \frac{1}{2} x\left|f^{\prime}(x)\right|,
$$


which, together with the doubling property, gives

$$
f(x) \geq C x\left|f^{\prime}(x)\right|
$$

for $x>2 x_{0}$. Hence, we obtain our assertion in the case $x_{0}=0$. If $x_{0}>0$, we observe that the function

$$
\left[x_{0}, 2 x_{0}\right] \ni x \mapsto \frac{x\left|f^{\prime}(x)\right|}{f(x)}
$$

is continuous and positive and thus bounded. This completes the proof.

Proposition 2.2. Let $f$ be a completely monotone function. Suppose that $-f^{\prime} \in$ $W L S C\left(\tau, c, x_{0}\right)$ for some $c \in(0,1], x_{0} \geq 0$ and $\tau \leq-1$. Then $f \in W L S C\left(1+\tau, c, x_{0}\right)$.

Analogously, if $-f^{\prime} \in W U S C\left(\tau, C, x_{0}\right)$ for some $C \geq 1, x_{0} \geq 0$ and $\tau \leq-1$, then $(f-$ $f(\infty)) \in \operatorname{WUSC}\left(\tau, C, x_{0}\right)$.

Proof. Let $\lambda>1$. For $y>x>x_{0}$, we have

$$
\begin{aligned}
f(\lambda x)-f(\lambda y)=-\int_{\lambda x}^{\lambda y} f^{\prime}(s) \mathrm{d} s & =-\lambda \int_{x}^{y} f^{\prime}(\lambda s) \mathrm{d} s \\
& \geq-c \lambda^{1+\tau} \int_{x}^{y} f^{\prime}(s) \mathrm{d} s=c \lambda^{1+\tau}(f(x)-f(y)) ;
\end{aligned}
$$

thus,

$$
f(\lambda x) \geq c \lambda^{1+\tau} f(x)+f(\lambda y)-c \lambda^{1+\tau} f(y) .
$$

Since $f$ is nonnegative and nonincreasing, we can take $y$ approaching infinity to get

$$
\begin{aligned}
f(\lambda x) & \geq c \lambda^{1+\tau} f(x)+\left(1-c \lambda^{1+\tau}\right) \lim _{y \rightarrow \infty} f(y) \\
& \geq c \lambda^{1+\tau} f(x),
\end{aligned}
$$

where in the last inequality we have also used that $1 \geq c \lambda^{1+\tau}$. The second part of the proposition can be proved in much the same way.

Proposition 2.3. Let $\phi$ be a Bernstein function with $\phi(0)=0$. Then $\phi \in W L S C\left(\alpha, c, x_{0}\right)$ for some $c \in(0,1], x_{0} \geq 0$ and $\alpha>0$ if and only if $\phi^{\prime} \in W L S C\left(\alpha-1, c^{\prime}, x_{0}\right)$ for some $c^{\prime} \in(0,1]$. Furthermore, if $\phi \in W L S C\left(\alpha, c, x_{0}\right)$, then there is $C \geq 1$ such that for all $x>x_{0}$,

$$
x \phi^{\prime}(x) \leq \phi(x) \leq C x \phi^{\prime}(x)
$$

Proof. Assume first that $\phi^{\prime} \in \operatorname{WLSC}\left(\alpha-1, c, x_{0}\right)$. Without loss of generality, we can assume $\phi^{\prime} \not \equiv 0$. We claim that (2.12) holds true. In view of (2.9), it is enough to show that there is $C \geq 1$ such that for all $x>x_{0}$,

$$
\phi(x) \leq C x \phi^{\prime}(x) .
$$

First, let us observe that, by the weak lower scaling property of $\phi^{\prime}$,

$$
\phi(x)-\phi\left(x_{0}\right)=\int_{x_{0}}^{x} \phi^{\prime}(s) \mathrm{d} s
$$




$$
\begin{aligned}
& \leq c^{-1} \phi^{\prime}(x) \int_{x_{0}}^{x}(s / x)^{-1+\alpha} \mathrm{d} s \\
& \leq \frac{1}{c \alpha} x \phi^{\prime}(x) .
\end{aligned}
$$

Thus, we get the assertion in the case $x_{0}=0$. If $x_{0}>0$, it is enough to show that there is $C>0$ such that for all $x>x_{0}$,

$$
x \phi^{\prime}(x) \geq C \text {. }
$$

Since $\phi^{\prime} \in \operatorname{WLSC}\left(\alpha-1, c, x_{0}\right)$, the function

$$
\left(x_{0}, \infty\right) \ni x \mapsto x \phi^{\prime}(x)
$$

is almost increasing. Hence, for $x \geq 2 x_{0}$ we have

$$
x \phi^{\prime}(x) \geq c 2 x_{0} \phi^{\prime}\left(2 x_{0}\right) .
$$

To conclude (2.14), we notice that $\phi^{\prime}(x)$ is positive and continuous in $\left[x_{0}, 2 x_{0}\right]$. Now, by (2.14) we get

$$
x \phi^{\prime}(x) \geq C \phi\left(x_{0}\right)
$$

for all $x>x_{0}$, which, together with (2.13), implies (2.12) and the scaling property of $\phi$ follows.

Now assume that $\phi \in \operatorname{WLSC}\left(\alpha, c, x_{0}\right)$. By monotonicity of $\phi^{\prime}$, for $0<s<t$,

$$
\frac{\phi(t x)-\phi(s x)}{\phi(x)} \leq \frac{x(t-s) \phi^{\prime}(s x)}{\phi(x)} .
$$

For $s=1$, by the lower scaling we get

$$
\frac{x(t-1) \phi^{\prime}(x)}{\phi(x)} \geq \frac{\phi(t x)}{\phi(x)}-1 \geq c t^{\alpha}-1,
$$

for all $x>x_{0}$. Thus, for $t=2^{1 / \alpha} c^{-1 / \alpha}$, we obtain that $x \phi^{\prime}(x) \gtrsim \phi(x)$ for all $x>x_{0}$. Invoking (2.9), we conclude (2.12). In particular, $\phi^{\prime}$ has the weak lower scaling property. This completes the proof.

Proposition 2.4. Let $\phi$ be a Bernstein function. Suppose that $-\phi^{\prime \prime} \in W U S C\left(\beta-2, C, x_{0}\right)$ for some $C \geq 1, x_{0} \geq 0$ and $\beta<1$. Then for all $x>x_{0}$,

$$
\phi^{\prime}(x) \leq \frac{C}{1-\beta} x\left(-\phi^{\prime \prime}(x)\right)+b,
$$

where $b$ is the drift term from the integral representation (2.11) of $\phi$.

Proof. Without loss of generality, we can assume $\phi^{\prime \prime} \not \equiv 0$. By the scaling property, for $x>x_{0}$ we have

$$
\frac{\phi^{\prime}(x)-b}{x\left(-\phi^{\prime \prime}(x)\right)}=\int_{x}^{\infty} \frac{t}{x} \frac{\left(-\phi^{\prime \prime}(t)\right)}{\left(-\phi^{\prime \prime}(x)\right)} \frac{\mathrm{d} t}{t} \leq C \int_{x}^{\infty}\left(\frac{t}{x}\right)^{-1+\beta} \frac{\mathrm{d} t}{t}=C \frac{1}{1-\beta},
$$

which concludes the proof. 
Remark 2.5. Let $\phi$ be a Bernstein function such that $\phi(0)=0$. Suppose that $-\phi^{\prime \prime} \in$ $\operatorname{WLSC}\left(\alpha-2, c, x_{0}\right)$, for some $c \in(0,1], x_{0} \geq 0$ and $\alpha \in(0,1]$. Since $\phi^{\prime}$ is completely monotone, by Proposition 2.2, $\phi^{\prime} \in \operatorname{WLSC}\left(\alpha-1, c, x_{0}\right)$. Therefore, by Proposition 2.3, we conclude that $\phi \in \operatorname{WLSC}\left(\alpha, c_{1}, x_{0}\right)$ for some $c_{1} \in(0,1]$.

Proposition 2.6. Let $f$ be a completely monotone function. Suppose that

$$
f \in W L S C\left(\alpha-1, c, x_{0}\right) \cap W U S C\left(\beta-1, C, x_{0}\right)
$$

for some $c \in(0,1], C \geq 1, x_{0} \geq 0$ and $0<\alpha \leq \beta<1$. Then

$$
-f^{\prime} \in W L S C\left(\alpha-2, c^{\prime}, x_{0}\right) \cap W U S C\left(\beta-2, C^{\prime}, x_{0}\right)
$$

for some $c^{\prime} \in(0,1]$ and $C^{\prime} \geq 1$.

Proof. By monotonicity of $-f^{\prime}$, for $0<s<t$,

$$
\frac{-x(t-s) f^{\prime}(t x)}{f(x)} \leq \frac{f(s x)-f(t x)}{f(x)} \leq \frac{-x(t-s) f^{\prime}(s x)}{f(x)} .
$$

Taking $s=1$ in the second inequality, the weak upper scaling property yields

$$
\frac{-x(t-1) f^{\prime}(x)}{f(x)} \geq 1-\frac{f(t x)}{f(x)} \geq 1-c t^{\beta-1},
$$

for all $x>x_{0}$. By selecting $t>1$ such that $c t^{\beta-1} \leq \frac{1}{2}$, we obtain $x\left(-f^{\prime}(x)\right) \gtrsim f(x)$ for $x>x_{0}$. Similarly, taking $t=1$ in the first inequality in (2.15), by the weak lower scaling property we get

$$
\frac{-x(1-s) f^{\prime}(x)}{f(x)} \leq \frac{f(s x)}{f(x)}-1 \leq c^{-1} s^{\alpha-1}-1,
$$

for all $x>x_{0} / s$. By selecting $0<s<1$ such that $s^{\alpha-1} \geq 2 c$, we obtain $x\left(-f^{\prime}(x)\right) \lesssim f(x)$ for $x>x_{0} / s$. Hence,

$$
f(x) \approx x\left(-f^{\prime}(x)\right)
$$

for all $x>x_{0} / s$. Therefore, lower and upper scaling properties follow from (2.16) and the scaling properties of $f$. This finishes the proof for the case $x_{0}=0$. If $x_{0}>0$, we notice that both $f$ ad $-f^{\prime}$ are positive and continuous; thus, at the possible expense of worsening the constants, we get (2.16) for all $x>x_{0}$.

Now, by combining Propositions 2.3 and 2.6, we immediately get the following corollary.

Corollary 2.7. Let $\phi$ be a Bernstein function such that $\phi(0)=0$. Suppose that

$$
\phi \in W L S C\left(\alpha, c, x_{0}\right) \cap W U S C\left(\beta, C, x_{0}\right)
$$

for some $c \in(0,1], C \geq 1, x_{0} \geq 0$ and $0<\alpha \leq \beta<1$. Then

$$
-\phi^{\prime \prime} \in W L S C\left(\alpha-2, c^{\prime}, x_{0}\right) \cap W U S C\left(\beta-2, C^{\prime}, x_{0}\right)
$$

for some $c^{\prime} \in(0,1]$ and $C^{\prime} \geq 1$. 
Lemma 2.8. Let $\phi$ be a Bernstein function. Suppose that $-\phi^{\prime \prime} \in W L S C\left(\alpha-2, c, x_{0}\right)$ for some $c \in(0,1], x_{0} \geq 0$ and $\alpha>0$. There is a constant $C>0$ such that for all $x>x_{0}$,

$$
C\left(-\phi^{\prime \prime}(x)\right) \leq \int_{(0,1 / x)} s^{2} \mu(d s) .
$$

Moreover, the constant $C$ depends only on $\alpha$ and $c$.

Proof. Let $f:(0, \infty) \rightarrow \mathbb{R}$ be a function defined as

$$
f(t)=\int_{(0, t)} s^{2} \mu(\mathrm{d} s) .
$$

We observe that, by the Fubini-Tonelli theorem, for $x>0$ we have

$$
\begin{aligned}
\mathcal{L} f(x) & =\int_{0}^{\infty} e^{-x t} \int_{(0, t)} s^{2} \mu(\mathrm{d} s) \mathrm{d} t \\
& =\int_{(0, \infty)} s^{2} \int_{s}^{\infty} e^{-x t} \mathrm{~d} t \mu(\mathrm{d} s)=x^{-1}\left(-\phi^{\prime \prime}(x)\right) .
\end{aligned}
$$

Since $f$ is nondecreasing, for any $s>0$,

$$
\begin{aligned}
-\phi^{\prime \prime}(x)=x \mathcal{L} f(x) & \geq \int_{s}^{\infty} e^{-t} f(t / x) \mathrm{d} t \\
& \geq e^{-s} f(s / x) .
\end{aligned}
$$

Hence, for any $u>2$,

$$
\begin{aligned}
-\phi^{\prime \prime}(x) & =\int_{0}^{u} e^{-s} f(s / x) \mathrm{d} s+\int_{u}^{\infty} e^{-s} f(s / x) \mathrm{d} s \\
& \leq f(u / x)+\int_{u}^{\infty} e^{-s / 2}\left(-\phi^{\prime \prime}(x / 2)\right) \mathrm{d} s .
\end{aligned}
$$

Therefore, setting $x=\lambda u>2 x_{0}$, by the weak lower scaling property of $-\phi^{\prime \prime}$,

$$
\begin{aligned}
f(1 / \lambda) & \geq-\phi^{\prime \prime}(u \lambda)-2 e^{-u / 2}\left(-\phi^{\prime \prime}(u \lambda / 2)\right) \\
& \geq\left(2^{\alpha-2} c-2 e^{-u / 2}\right)\left(-\phi^{\prime \prime}(u \lambda / 2)\right) .
\end{aligned}
$$

At this stage, we select $u>2$ such that

$$
2^{\alpha-2} c-2 e^{-u / 2} \geq 2^{-2} c .
$$

Then again, by the weak lower scaling property of $-\phi^{\prime \prime}$, for $\lambda>x_{0}$,

$$
f(1 / \lambda) \geq c 2^{-2}\left(-\phi^{\prime \prime}(u \lambda / 2)\right) \geq c^{2} 2^{-\alpha} u^{\alpha-2}\left(-\phi^{\prime \prime}(\lambda)\right)
$$

which ends the proof. 
Lemma 2.9. Let $\phi$ be a Bernstein function. Suppose that $-\phi^{\prime \prime} \in \operatorname{WLSC}\left(\alpha-2, c, x_{0}\right)$ for some $c \in(0,1], x_{0} \geq 0$ and $\alpha>0$. Then there exists a complete Bernstein function $f$ such that $f \approx \phi$ for $x>0$ and $-f^{\prime \prime} \approx-\phi^{\prime \prime}$ for $x>x_{0}$.

Proof. Let us define

$$
f(\lambda)=a+b \lambda+\int_{0}^{\infty} \frac{\lambda u}{\lambda u+1} \mu(\mathrm{d} u) .
$$

By [48, Theorem 6.2 (vi)] the function $f$ is a complete Bernstein function. Since for $y>0$,

$$
\frac{y}{y+1} \approx\left(1-e^{-y}\right)
$$

we get $f(\lambda) \approx \phi(\lambda)$. Moreover,

$$
f^{\prime \prime}(\lambda)=-2 \int_{0}^{\infty} \frac{u^{2}}{(\lambda u+1)^{3}} \mu(\mathrm{d} u) .
$$

Hence, by Lemma 2.8 we obtain $-f^{\prime \prime}(\lambda) \approx-\phi^{\prime \prime}(\lambda)$ for $\lambda>x_{0}$.

\section{Asymptotic behaviour of densities}

Let $\mathbf{T}=\left(T_{t}: t \geq 0\right)$ be a subordinator with the Lévy-Khintchine exponent $\psi$ and the Laplace exponent $\phi$. Since $\phi$ is a Bernstein function, it admits the integral representation (2.11). As it may be easily checked (see, e.g., [48, Proposition 3.6]), we have $\mu=\nu, a=0$ and $\psi(\xi)=\phi(-i \xi)$. In particular, $\phi(0)=0$.

In this section we study the asymptotic behaviour of the probability density of $T_{t}$. In the whole section we assume that $\phi^{\prime \prime} \not \equiv 0$; otherwise, $T_{t}=b t$ is deterministic. The main result is Theorem 3.3. Let us start by showing an estimate on the real part of the complex extension $\phi$.

Lemma 3.1. Suppose that $-\phi^{\prime \prime} \in \operatorname{WLSC}\left(\alpha-2, c, x_{0}\right)$ for some $c \in(0,1], x_{0} \geq 0$ and $\alpha>0$. Then there exists $C>0$ such that for all $w>x_{0}$ and $\lambda \in \mathbb{R}$,

$$
\Re(\phi(w+i \lambda)-\phi(w)) \geq C \lambda^{2}\left(-\phi^{\prime \prime}(|\lambda| \vee w)\right) .
$$

Proof. By the integral representation (2.11), for $\lambda \in \mathbb{R}$ we have

$$
\Re(\phi(w+i \lambda)-\phi(w))=\int_{(0, \infty)}(1-\cos (\lambda s)) e^{-w s} \nu(\mathrm{d} s) .
$$

In particular,

$$
\Re(\phi(w+i \lambda)-\phi(w))=\Re(\phi(w-i \lambda)-\phi(w)) .
$$

Thus, it is sufficient to consider $\lambda>0$. We can estimate

$$
\begin{aligned}
\Re(\phi(w+i \lambda)-\phi(w)) & \geq \int_{(0,1 / \lambda)}(1-\cos (\lambda s)) e^{-w s} \nu(\mathrm{d} s) \\
& \gtrsim \lambda^{2} \int_{(0,1 / \lambda)} s^{2} e^{-w s} \nu(\mathrm{d} s) .
\end{aligned}
$$


Due to Lemma 2.8, we obtain, for $\lambda \geq w$,

$$
\Re(\phi(w+i \lambda)-\phi(w)) \gtrsim \lambda^{2} \int_{(0,1 / \lambda)} s^{2} \nu(\mathrm{d} s) \gtrsim \lambda^{2}\left(-\phi^{\prime \prime}(\lambda)\right) .
$$

If $w>\lambda>0$, then, by (3.1), we have

$$
\begin{aligned}
\Re(\phi(w+i \lambda)-\phi(w)) & \gtrsim \lambda^{2} \int_{(0,1 / w)} s^{2} e^{-w s} \nu(\mathrm{d} s) \\
& \geq e^{-1} \lambda^{2} \int_{(0,1 / w)} s^{2} \nu(\mathrm{d} s),
\end{aligned}
$$

which, by Lemma 2.8, completes the proof.

Remark 3.2. Suppose that $-\phi^{\prime \prime} \in \operatorname{WLSC}\left(\alpha-2, c, x_{0}\right)$ for some $c \in(0,1], x_{0} \geq 0$ and $\alpha>0$. Since

$$
K(1 / x) \leq e x^{2}\left(-\phi^{\prime \prime}(x)\right)
$$

by Lemma 2.8 we obtain

$$
C x^{2}\left(-\phi^{\prime \prime}(x)\right) \leq K(1 / x) \leq e x^{2}\left(-\phi^{\prime \prime}(x)\right)
$$

for all $x>x_{0}$.

Theorem 3.3. Let $\mathbf{T}$ be a subordinator with the Laplace exponent $\phi$. Suppose that $-\phi^{\prime \prime} \in$ $\operatorname{WLSC}\left(\alpha-2, c, x_{0}\right)$ for some $c \in(0,1], x_{0} \geq 0$ and $\alpha>0$. Then the probability distribution of $T_{t}$ is absolutely continuous for all $t>0$. If we denote its density by $p(t, \cdot)$, then for each $\epsilon>0$ there is $M_{0}>0$ such that

$$
\left|p\left(t, t \phi^{\prime}(w)\right) \sqrt{2 \pi t\left(-\phi^{\prime \prime}(w)\right)} \exp \left\{t\left(\phi(w)-w \phi^{\prime}(w)\right)\right\}-1\right| \leq \epsilon,
$$

provided that $w>x_{0}$ and $t w^{2}\left(-\phi^{\prime \prime}(w)\right)>M_{0}$.

Proof. Let $x=t \phi^{\prime}(w)$ and $M>0$. We first show that

$$
p(t, x)=\frac{1}{2 \pi} \cdot \frac{e^{-t \Phi(x / t, 0)}}{\sqrt{t\left(-\phi^{\prime \prime}(w)\right)}} \int_{\mathbb{R}} \exp \left\{-t\left(\Phi\left(\frac{x}{t}, \frac{u}{\sqrt{t\left(-\phi^{\prime \prime}(w)\right)}}\right)-\Phi\left(\frac{x}{t}, 0\right)\right)\right\} \mathrm{d} u,
$$

provided that $w>x_{0}$ and $t w^{2}\left(-\phi^{\prime \prime}(w)\right)>M$, where for $\lambda \in \mathbb{R}$ we have set

$$
\Phi(x / t, \lambda)=\phi(w+i \lambda)-\frac{x}{t}(w+i \lambda)
$$

To do so, let us recall that

$$
\mathbb{E}\left(e^{-\lambda T_{t}}\right)=e^{-t \phi(\lambda)}, \quad \lambda \geq 0 .
$$

Thus, by Mellin's inversion formula, if the limit

$$
\lim _{L \rightarrow \infty} \frac{1}{2 \pi i} \int_{w-i L}^{w+i L} e^{-t \phi(\lambda)+\lambda x} \mathrm{~d} \lambda \text { exists, }
$$


then the probability distribution of $T_{t}$ has a density $p(t, \cdot)$ and

$$
p(t, x)=\lim _{L \rightarrow \infty} \frac{1}{2 \pi i} \int_{w-i L}^{w+i L} e^{-t \phi(\lambda)+\lambda x} \mathrm{~d} \lambda .
$$

Therefore, our task is to justify the statement (3.4). For $L>0$, we write

$$
\frac{1}{2 \pi i} \int_{w-i L}^{w+i L} e^{-t \phi(\lambda)+\lambda x} \mathrm{~d} \lambda=\frac{1}{2 \pi} \int_{-L}^{L} e^{-t \Phi(x / t, \lambda)} \mathrm{d} \lambda .
$$

By the change of variables

$$
\lambda=\frac{u}{\sqrt{t\left(-\phi^{\prime \prime}(w)\right)}}
$$

we obtain

$$
\begin{aligned}
& \int_{-L}^{L} e^{-t \Phi(x / t, \lambda)} \mathrm{d} \lambda=e^{-t \Phi(x / t, 0)} \int_{-L}^{L} \exp \{-t(\Phi(x / t, \lambda)-\Phi(x / t, 0))\} \mathrm{d} \lambda \\
& =\frac{e^{-t \Phi(x / t, 0)}}{\sqrt{t\left(-\phi^{\prime \prime}(w)\right)}} \times \\
& \int_{-L \sqrt{t\left(-\phi^{\prime \prime}(w)\right)}}^{L \sqrt{t\left(-\phi^{\prime \prime}(w)\right)}} \exp \left\{-t\left(\Phi\left(\frac{x}{t}, \frac{u}{\sqrt{t\left(-\phi^{\prime \prime}(w)\right)}}\right)-\Phi\left(\frac{x}{t}, 0\right)\right)\right\} \mathrm{d} u .
\end{aligned}
$$

Let us note here that $-\phi^{\prime \prime}$ is nonincreasing and integrable at infinity; thus, we in fact have $\alpha \leq 1$. We claim that there is $C>0$ not depending on $M$, such that for all $u \in \mathbb{R}$,

$$
t \Re\left(\Phi\left(\frac{x}{t}, \frac{u}{\sqrt{t\left(-\phi^{\prime \prime}(w)\right)}}\right)-\Phi\left(\frac{x}{t}, 0\right)\right) \geq C\left(u^{2} \wedge\left(|u|^{\alpha} M^{1-\alpha / 2}\right)\right),
$$

provided that $w>x_{0}$ and $t w^{2}\left(-\phi^{\prime \prime}(w)\right)>M$. Indeed, by (3.3) and Lemma 3.1, for $w>x_{0}$ we get

$$
t \Re\left(\Phi\left(\frac{x}{t}, \frac{u}{\sqrt{t\left(-\phi^{\prime \prime}(w)\right)}}\right)-\Phi\left(\frac{x}{t}, 0\right)\right) \gtrsim \frac{|u|^{2}}{\phi^{\prime \prime}(w)} \phi^{\prime \prime}\left(\frac{|u|}{\sqrt{t\left(-\phi^{\prime \prime}(w)\right)}} \vee w\right) .
$$

We next estimate the right-hand side of (3.6). If $|u| \leq w \sqrt{t\left(-\phi^{\prime \prime}(w)\right)}$, then

$$
\frac{|u|^{2}}{\phi^{\prime \prime}(w)} \phi^{\prime \prime}\left(\frac{|u|}{\sqrt{t\left(-\phi^{\prime \prime}(w)\right)}} \vee w\right)=|u|^{2} \text {. }
$$

Otherwise, since $-\phi^{\prime \prime} \in \operatorname{WLSC}\left(\alpha-2, c, x_{0}\right)$, we obtain

$$
\begin{aligned}
\frac{|u|^{2}}{\phi^{\prime \prime}(w)} \phi^{\prime \prime}\left(\frac{|u|}{\sqrt{t\left(-\phi^{\prime \prime}(w)\right)}} \vee w\right) & \geq c|u|^{2}\left(\frac{|u|}{\sqrt{t w^{2}\left(-\phi^{\prime \prime}(w)\right)}}\right)^{-2+\alpha} \\
& =c|u|^{\alpha}\left(t w^{2}\left(-\phi^{\prime \prime}(w)\right)\right)^{1-\alpha / 2} \\
& \geq c M^{1-\alpha / 2}|u|^{\alpha}
\end{aligned}
$$


Hence, we deduce (3.5). To finish the proof of (3.4), we invoke the dominated convergence theorem. Consequently, by Mellin's inversion formula we obtain (3.2).

Our next task is to show that for each $\epsilon>0$ there is $M_{0}>0$ such that

$$
\left|\int_{\mathbb{R}} \exp \left\{-t\left(\Phi\left(\frac{x}{t}, \frac{u}{\sqrt{t\left(-\phi^{\prime \prime}(w)\right)}}\right)-\Phi\left(\frac{x}{t}, 0\right)\right)\right\} \mathrm{d} u-\int_{\mathbb{R}} e^{-\frac{1}{2} u^{2}} \mathrm{~d} u\right| \leq \epsilon,
$$

provided that $w>x_{0}$ and $t w^{2}\left(-\phi^{\prime \prime}(w)\right)>M_{0}$. In view of (3.5), by taking $M_{0}>1$ sufficiently large, we get

$$
\left|\int_{|u| \geq M_{0}^{1 / 4}} \exp \left\{-t\left(\Phi\left(\frac{x}{t}, \frac{u}{\sqrt{t\left(-\phi^{\prime \prime}(w)\right)}}\right)-\Phi\left(\frac{x}{t}, 0\right)\right)\right\} \mathrm{d} u\right| \leq \int_{|u| \geq M_{0}^{1 / 4}} e^{-C|u|^{\alpha}} \mathrm{d} u \leq \epsilon
$$

and

$$
\int_{|u| \geq M_{0}^{1 / 4}} e^{-\frac{1}{2} u^{2}} \mathrm{~d} u \leq \epsilon
$$

Next, we claim that there is $C>0$ such that

$$
\left.\left.\left|t\left(\Phi\left(\frac{x}{t}, \frac{u}{\sqrt{t\left(-\phi^{\prime \prime}(w)\right)}}\right)-\Phi\left(\frac{x}{t}, 0\right)\right)-\frac{1}{2}\right| u\right|^{2}|\leq C| u\right|^{3} M_{0}^{-\frac{1}{2}}
$$

Indeed, since

$$
\partial_{\lambda} \Phi\left(\frac{x}{t}, 0\right)=0
$$

by Taylor's formula, we get

$$
\begin{aligned}
\left.\left|t\left(\Phi\left(\frac{x}{t}, \frac{u}{\sqrt{t\left(-\phi^{\prime \prime}(w)\right)}}\right)-\Phi\left(\frac{x}{t}, 0\right)\right)-\frac{1}{2}\right| u\right|^{2} \mid & =\left.\left|\frac{1}{2} \partial_{\lambda}^{2} \Phi\left(\frac{x}{t}, \xi\right) \frac{|u|^{2}}{-\phi^{\prime \prime}(w)}-\frac{1}{2}\right| u\right|^{2} \mid \\
& =\frac{|u|^{2}}{2\left|\phi^{\prime \prime}(w)\right|}\left|\phi^{\prime \prime}(w+i \xi)-\phi^{\prime \prime}(w)\right|
\end{aligned}
$$

where $\xi$ is some number satisfying

$$
|\xi| \leq \frac{|u|}{\sqrt{t\left(-\phi^{\prime \prime}(w)\right)}}
$$

Observe that

$$
\begin{aligned}
\left|\phi^{\prime \prime}(w+i \xi)-\phi^{\prime \prime}(w)\right| & \leq \int_{(0, \infty)} s^{2} e^{-w s}\left|e^{-i \xi s}-1\right| \nu(\mathrm{d} s) \\
& \leq 2|\xi| \int_{(0, \infty)} s^{3} e^{-w s} \nu(\mathrm{d} s)=2|\xi| \phi^{\prime \prime \prime}(w)
\end{aligned}
$$

Since $-\phi^{\prime \prime}$ is a nonincreasing function with the weak lower scaling property, it is doubling. Thus, by Proposition 2.1, for $w>x_{0}$,

$$
-\phi^{\prime \prime}(w) \gtrsim w \phi^{\prime \prime \prime}(w),
$$


which together with (3.12) give

$$
\begin{aligned}
\left|\phi^{\prime \prime}(w+i \xi)-\phi^{\prime \prime}(w)\right| & \leq C \frac{|u|}{\sqrt{t\left(-\phi^{\prime \prime}(w)\right)}} \cdot \frac{-\phi^{\prime \prime}(w)}{w} \\
& \leq C M_{0}^{-\frac{1}{2}}|u|\left(-\phi^{\prime \prime}(w)\right),
\end{aligned}
$$

whenever $t w^{2}\left(-\phi^{\prime \prime}(w)\right)>M_{0}$. Now, (3.10) easily follows by (3.13) and (3.11).

Finally, since for any $z \in \mathbb{C}$,

$$
\left|e^{z}-1\right| \leq|z| e^{|z|}
$$

by (3.10) we obtain

$$
\begin{gathered}
\left|\int_{|u| \leq M_{0}^{1 / 4}} \exp \left\{-t\left(\Phi\left(\frac{x}{t}, \frac{u}{\sqrt{t\left(-\phi^{\prime \prime}(w)\right)}}\right)-\Phi\left(\frac{x}{t}, 0\right)\right)\right\}-e^{-\frac{1}{2}|u|^{2}} \mathrm{~d} u\right| \\
\leq C M_{0}^{-\frac{1}{2}} \int_{|u| \leq M_{0}^{1 / 4}} \exp \left\{-\frac{1}{2}|u|^{2}+C M_{0}^{-\frac{1}{2}}|u|^{3}\right\}|u|^{3} \mathrm{~d} u \leq \epsilon
\end{gathered}
$$

provided that $M_{0}$ is sufficiently large, which, together with (3.8) and (3.9), completes the proof of (3.7) and the theorem follows.

Remark 3.4. If $x_{0}=0$, then the constant $M_{0}$ in Theorem 3.3 depends only on $\alpha$ and $c$. If $x_{0}>0$, it also depends on

$$
\sup _{x \in\left[x_{0}, 2 x_{0}\right]} \frac{x \phi^{\prime \prime \prime}(x)}{-\phi^{\prime \prime}(x)} .
$$

By Theorem 3.3, we immediately get the following corollaries.

Corollary 3.5. Suppose that $-\phi^{\prime \prime} \in \operatorname{WLSC}\left(\alpha-2, c, x_{0}\right)$ for some $c \in(0,1], x_{0} \geq 0$ and $\alpha>0$. Then there is $M_{0}>0$ such that

$$
p(t, x) \approx \frac{1}{\sqrt{t\left(-\phi^{\prime \prime}(w)\right)}} \exp \left\{-t\left(\phi(w)-w \phi^{\prime}(w)\right)\right\},
$$

uniformly on the set

$$
\left\{(t, x) \in \mathbb{R}_{+} \times \mathbb{R}: t b<x<t \phi^{\prime}\left(x_{0}^{+}\right) \text {and } t w^{2}\left(-\phi^{\prime \prime}(w)\right)>M_{0}\right\},
$$

where $w=\left(\phi^{\prime}\right)^{-1}(x / t)$.

Corollary 3.6. Suppose that $-\phi^{\prime \prime} \in \operatorname{WLSC}\left(\alpha-2, c, x_{0}\right)$ for some $c \in(0,1], x_{0} \geq 0$ and $\alpha>0$. Assume also that $b=0$. Then for any $x>0$,

$$
\lim _{t \rightarrow \infty} p(t, x) \sqrt{t\left(-\phi^{\prime \prime}(w)\right)} \exp \left\{t\left(\phi(w)-w \phi^{\prime}(w)\right)\right\}=(2 \pi)^{-1 / 2},
$$

where $w=\left(\phi^{\prime}\right)^{-1}(x / t)$.

By imposing on $-\phi^{\prime \prime}$ an additional condition of the weak upper scaling, we can further simplify the description of the set where the sharp estimates on $p(t, x)$ hold. 
Corollary 3.7. Suppose that $\phi \in \operatorname{WLSC}\left(\alpha, c, x_{0}\right) \cap \operatorname{WUSC}\left(\beta, C, x_{0}\right)$ for some $c \in(0,1]$, $C \geq 1, x_{0} \geq 0$ and $0<\alpha \leq \beta<1$. Assume also that $b=0$. Then there is $\delta>0$ such that

$$
p(t, x) \approx \frac{1}{\sqrt{t\left(-\phi^{\prime \prime}(w)\right)}} \exp \left\{-t\left(\phi(w)-w \phi^{\prime}(w)\right)\right\},
$$

uniformly on the set

$$
\left\{(t, x) \in \mathbb{R}_{+} \times \mathbb{R}: 0<x \phi^{-1}(1 / t)<\delta, \text { and } 0 \leq t \phi\left(x_{0}\right) \leq 1\right\}
$$

where $w=\left(\phi^{\prime}\right)^{-1}(x / t)$.

Proof. By Proposition 2.3, there is $C_{1} \geq 1$ such that for all $u>x_{0}$,

$$
\phi(u) \leq C_{1} u \phi^{\prime}(u)
$$

thus, for $(t, x)$ belonging to the set (3.14),

$$
\begin{aligned}
\frac{x}{t}<\delta \frac{1}{t \phi^{-1}(1 / t)} & =\delta \frac{\phi\left(\phi^{-1}(1 / t)\right)}{\phi^{-1}(1 / t)} \\
& \leq C_{1} \delta \phi^{\prime}\left(\phi^{-1}(1 / t)\right) .
\end{aligned}
$$

By Proposition 2.3, $\phi^{\prime} \in \mathrm{WLSC}\left(-1+\alpha, c, x_{0}\right)$; hence, for all $D \geq 1$,

$$
\phi^{\prime}\left(D \phi^{-1}(1 / t)\right) \geq c D^{-1+\alpha} \phi^{\prime}\left(\phi^{-1}(1 / t)\right) .
$$

By taking $\delta$ sufficiently small, we get

$$
D=\left(\frac{c}{C_{1} \delta}\right)^{\frac{1}{1-\alpha}} \geq 1
$$

thus, by (3.15), we obtain

$$
\frac{x}{t}<\phi^{\prime}\left(D \phi^{-1}(1 / t)\right)
$$

which implies that

$$
w=\left(\phi^{\prime}\right)^{-1}(x / t)>D \phi^{-1}(1 / t) .
$$

In particular, $w>x_{0}$. On the other hand, by Propositions 2.3 and 2.4 , there is $c_{1} \in(0,1]$ such that

$$
t w^{2}\left(-\phi^{\prime \prime}(w)\right) \geq c_{1} t \phi(w)
$$

By Remark 2.5, $\phi \in \operatorname{WLSC}\left(\alpha, c_{2}, x_{0}\right)$ for some $c_{2} \in(0,1]$. Therefore,

$$
t \phi(w)=\frac{\phi(w)}{\phi\left(\phi^{-1}(1 / t)\right)} \geq c_{2}\left(\frac{w}{\phi^{-1}(1 / t)}\right)^{\alpha},
$$

which, together with (3.16), gives

$$
t w^{2}\left(-\phi^{\prime \prime}(w)\right) \gtrsim \delta^{-\frac{\alpha}{1-\alpha}}>M_{0}
$$

for $\delta$ sufficiently small. Hence, by Corollary 3.5, we conclude the proof. 
The following proposition provides a sufficient condition on the measure $\nu$ that entails the weak lower scaling property of $-\phi^{\prime \prime}$ and allows us to apply Theorem 3.3.

Proposition 3.8. Suppose that there are $x_{0} \geq 0, C \geq 1$ and $\alpha>0$ such that for all $0<r<1 / x_{0}$ and $0<\lambda \leq 1$,

$$
\nu((r, \infty)) \leq C \lambda^{\alpha} \nu((\lambda r, \infty))
$$

Then $-\phi^{\prime \prime} \in \operatorname{WLSC}\left(\alpha-2, c, x_{0}\right)$ for some $c \in(0,1]$.

Proof. Let us first notice that by the Fubini-Tonelli theorem,

$$
\begin{aligned}
h(r) & =r^{-2} \int_{(0, \infty)} \min \left\{r^{2}, s^{2}\right\} \nu(\mathrm{d} s) \\
& =r^{-2} \int_{0}^{r} t \nu((t, \infty)) \mathrm{d} t .
\end{aligned}
$$

Thus, by (3.17), for all $0<r<1 / x_{0}$ and $0<\lambda \leq 1$,

$$
\begin{aligned}
C \lambda^{\alpha} h(\lambda r) & =\frac{2 C \lambda^{\alpha}}{r^{2}} \int_{0}^{r} t \nu((\lambda t, \infty)) \mathrm{d} t \\
& \geq \frac{2}{r^{2}} \int_{0}^{r} t \nu((t, \infty)) \mathrm{d} t \\
& =h(r) .
\end{aligned}
$$

Hence, by [23, Lemma 2.3], there is $C^{\prime} \geq 1$ such that for all $0<r<1 / x_{0}$,

$$
K(r) \leq h(r) \leq C^{\prime} K(r)
$$

The integral representation of $\phi$ entails that

$$
e^{-1} x^{-2} K(1 / x) \leq-\phi^{\prime \prime}(x) \leq e^{2} 2^{-2} x^{-2} h(1 / x), \quad x>0
$$

thus, by (3.19), we obtain

$$
-\phi^{\prime \prime}(x) \approx x^{-2} h(1 / x)
$$

for all $x>x_{0}$. Now, the weak lower scaling property of $-\phi^{\prime \prime}$ is a consequence of (3.18).

\section{Estimates on the density}

Let $\mathbf{T}=\left(T_{t}: t \geq 0\right)$ be a subordinator with the Lévy-Khintchine exponent $\psi$ and the Laplace exponent $\phi$. In this section we always assume that $-\phi^{\prime \prime} \in \operatorname{WLSC}\left(\alpha-2, c, x_{0}\right)$ for some $c \in(0,1], x_{0} \geq 0$ and $\alpha \in(0,1]$. In particular, by Theorem 3.3, the probability distribution of $T_{t}$ has a density $p(t, \cdot)$. To express the majorant on $p(t, \cdot)$, it is convenient to set

$$
\varphi(x)=x^{2}\left(-\phi^{\prime \prime}(x)\right), \quad x>0
$$


Obviously, $\varphi \in \operatorname{WLSC}\left(\alpha, c, x_{0}\right)$. Let $\varphi^{-1}$ denote the generalised inverse function defined as

$$
\varphi^{-1}(x)=\sup \left\{r>0: \varphi^{*}(r)=x\right\}
$$

where

$$
\varphi^{*}(r)=\sup _{0<x \leq r} \varphi(x)
$$

We start by showing comparability between the two concentration functions $K$ and $h$ defined in (2.3) and (2.4), respectively.

Proposition 4.1. Suppose that $-\phi^{\prime \prime} \in \operatorname{WLSC}\left(\alpha-2, c, x_{0}\right)$ for some $c \in(0,1], x_{0} \geq 0$ and $\alpha>0$. Then there is $C \geq 1$ such that for all $0<r<1 / x_{0}$,

$$
K(r) \leq h(r) \leq C K(r)
$$

Proof. Since $h(r) \geq K(r)$, it is enough to show that for some $C \geq 1$ and $0<r<1 / x_{0}$,

$$
h(r) \leq C K(r) \text {. }
$$

In view of $(2.5)$, we have

$$
h(r)=2 \int_{r}^{\infty} K(s) \frac{\mathrm{d} s}{s}=2 \int_{r}^{1 / x_{0}} K(s) \frac{\mathrm{d} s}{s}+2 \int_{1 / x_{0}}^{\infty} K(s) \frac{\mathrm{d} s}{s} .
$$

Let us consider the first term on the right-hand side of (4.1). By Remark 3.2 we have $K(r) \approx \varphi(1 / r)$, for $0<r<1 / x_{0}$, which implies

$$
\int_{r}^{1 / x_{0}} K(s) \frac{\mathrm{d} s}{s} \lesssim K(r), \quad 0<r<1 / x_{0} .
$$

This finishes the proof in the case $x_{0}=0$. If $x_{0}>0$, then, for $1 /\left(2 x_{0}\right) \leq r<1 / x_{0}$, we have

$$
K(r) \gtrsim \varphi(1 / r) \gtrsim \varphi\left(x_{0}\right)>0 .
$$

Hence, $K(r) \gtrsim 1$ for all $0<r<1 / x_{0}$. Since the second term on the right-hand side of (4.1) is constant, the proof is completed.

Let us notice that by (2.6), Proposition 4.1 and Remark 3.2, we have

$$
\psi^{*}(x) \approx h(1 / x) \approx K(1 / x) \approx \varphi(x)
$$

for all $x>x_{0}$. In particular, there is $c_{1} \in(0,1] \operatorname{such}$ that $\psi^{*} \in \operatorname{WLSC}\left(\alpha, c_{1}, x_{0}\right)$. Moreover,

$$
\begin{aligned}
\psi^{*}(x) \lesssim K(1 / x) & =x^{2} \int_{(0,1 / x)} s^{2} \nu(\mathrm{d} s) \\
& \lesssim \int_{(0,1 / x)}(1-\cos s x) \nu(\mathrm{d} s)
\end{aligned}
$$

thus, for all $x>x_{0}$,

$$
\psi^{*}(x) \lesssim \Re \psi(x)
$$


Since for $\lambda \geq 1$ and $x>0$,

$$
\varphi(\lambda x) \leq \lambda^{2} \varphi(x)
$$

we get

$$
\varphi^{*}(\lambda x) \leq \lambda^{2} \varphi^{*}(x)
$$

Proposition 4.2. Suppose that $-\phi^{\prime \prime} \in \operatorname{WLSC}\left(\alpha-2, c, x_{0}\right)$ for some $c \in(0,1], x_{0} \geq 0$ and $\alpha>0$. Then for all $r>2 h\left(1 / x_{0}\right)$,

$$
\frac{1}{h^{-1}(r)} \approx \psi^{-1}(r)
$$

Furthermore, there is $C \geq 1$ such that for all $\lambda \geq 1$ and $r>2 h\left(1 / x_{0}\right)$,

$$
\psi^{-1}(\lambda r) \leq C \lambda^{1 / \alpha} \psi^{-1}(r) .
$$

Proof. Using (2.6), we immediately get

$$
\frac{1}{h^{-1}(r / 2)} \leq \psi^{-1}(r) \leq \frac{1}{h^{-1}(24 r)}
$$

for all $r>0$. On the other hand, by Proposition 4.1 and [23, Lemma 2.3], there is $C \geq 1$ such that for all $\lambda \geq 1$ and $r>h\left(1 / x_{0}\right)$,

$$
\frac{1}{h^{-1}(\lambda r)} \leq C \lambda^{1 / \alpha} \frac{1}{h^{-1}(r)}
$$

Hence, for $r>2 h\left(1 / x_{0}\right)$,

$$
C^{-1} 2^{-1 / \alpha} \frac{1}{h^{-1}(r)} \leq \psi^{-1}(r) \leq C(24)^{1 / \alpha} \frac{1}{h^{-1}(r)},
$$

proving (4.6). The weak upper scaling property of $\psi^{-1}$ is a consequence of (4.7) and (4.8).

Proposition 4.3. Suppose that $-\phi^{\prime \prime} \in \mathrm{WLSC}\left(\alpha-2, c, x_{0}\right)$ for some $c \in(0,1], x_{0} \geq 0$ and $\alpha>0$. Then for all $x>x_{0}$,

$$
\psi^{*}(x) \approx \varphi^{*}(x)
$$

and for all $r>\varphi\left(x_{0}\right)$,

$$
\psi^{-1}(r) \approx \varphi^{-1}(r) .
$$

Furthermore, there is $C \geq 1$ such that for all $\lambda \geq 1$ and $r>\varphi\left(x_{0}\right)$,

$$
\varphi^{-1}(\lambda r) \leq C \lambda^{1 / \alpha} \varphi^{-1}(r) .
$$

Proof. We start by showing that there is $C \geq 1$ such that for all $x>x_{0}$,

$$
C^{-1} \psi^{*}(x) \leq \varphi^{*}(x) \leq C \psi^{*}(x) .
$$


The first inequality in (4.11) immediately follows from (4.2). If $x_{0}=0$, then the second inequality is also the consequence of (4.2). In the case $x_{0}>0$, we observe that for $x>x_{0}$, we have

$$
\begin{aligned}
\varphi^{*}(x) & =\max \left\{\sup _{0<y \leq x_{0}} \varphi(y), \sup _{x_{0} \leq y \leq x} \varphi(y)\right\} \\
& \lesssim \max \left\{\varphi^{*}\left(x_{0}\right), \psi^{*}(x)\right\} \\
& \leq\left(1+\frac{\varphi^{*}\left(x_{0}\right)}{\psi^{*}\left(x_{0}\right)}\right) \psi^{*}(x)
\end{aligned}
$$

proving (4.11).

Now, using (4.11), we easily get

$$
\psi^{-1}\left(C^{-1} r\right) \leq \varphi^{-1}(r) \leq \psi^{-1}(C r)
$$

for all $r>C \psi^{*}\left(x_{0}\right)$. Hence, by Proposition 4.2,

$$
\varphi^{-1}(r) \approx \psi^{-1}(r)
$$

for $r>C \max \left\{\psi^{*}\left(x_{0}\right), 2 h\left(1 / x_{0}\right)\right\}$. Finally, since both $\psi^{-1}$ and $\varphi^{-1}$ are positive and continuous, at the possible expense of worsening the constant, we can extend the area of comparability to conclude (4.10). Now, the scaling property of $\varphi^{-1}$ follows by (4.10) and Proposition 4.2.

Remark 4.4. Note that, alternatively, one can define the (left-sided) generalised inverse

$$
\varphi_{-1}(x)=\inf \left\{r>0: \varphi_{*}(r)=x\right\},
$$

where

$$
\varphi_{*}(r)=\inf _{r \leq x} \varphi(x)
$$

In such a case we have

$$
\varphi_{*}\left(\varphi_{-1}(s)\right)=s, \quad \text { and } \quad \varphi_{-1}\left(\varphi_{*}(s)\right) \leq s .
$$

Clearly, for all $x>0$,

$$
\varphi_{*}(x) \leq \varphi(x) \leq \varphi^{*}(x)
$$

Let $u>x_{0}$ and set

$$
r_{0}=\inf \left\{r>0: \varphi^{*}(r)=u\right\} .
$$

By Proposition 4.3, $\varphi^{*} \in \mathrm{WLSC}\left(\alpha, c, x_{0}\right)$ for some $c \in(0,1]$ and $x_{0} \geq 0$. Thus, for $\lambda>c^{-1 / \alpha}$, we get $\varphi^{*}\left(\lambda r_{0}\right)>\varphi^{*}\left(r_{0}\right)$. It follows that for all $u>x_{0}$,

$$
\begin{aligned}
\sup \left\{r>0: \varphi^{*}(r)=u\right\} & \leq \lambda \inf \left\{r>0: \varphi^{*}(r)=u\right\} \\
& \leq \lambda \inf \left\{r>0: \varphi_{*}(r)=u\right\} .
\end{aligned}
$$

Thus, for all $r>x_{0}$,

$$
\varphi^{-1}\left(\varphi^{*}(r)\right) \lesssim r
$$


Corollary 4.5. Suppose that $-\phi^{\prime \prime} \in \operatorname{WLSC}\left(\alpha-2, c, x_{0}\right)$ for some $c \in(0,1], x_{0} \geq 0$ and $\alpha>0$. Then there is $C>0$ such that for all $x>x_{0}$,

$$
\left(\phi(x)-x \phi^{\prime}(x)\right) \leq C \varphi(x) .
$$

Proof. Suppose $x_{0}>0$. We have

$$
\left(\phi(x)-x \phi^{\prime}(x)\right)-\left(\phi\left(x_{0}\right)-x_{0} \phi^{\prime}\left(x_{0}^{+}\right)\right)=\int_{x_{0}}^{x} \varphi(u) \frac{\mathrm{d} u}{u}=\int_{x_{0} / x}^{1} \varphi(x u) \frac{\mathrm{d} u}{u},
$$

where

$$
\phi^{\prime}\left(x_{0}^{+}\right)=\lim _{x \rightarrow x_{0}^{+}} \phi^{\prime}(x) .
$$

By the weak lower scaling property of $\varphi$, for any $x_{0} / x<u \leq 1$, we have

$$
\varphi(x) \geq c u^{-\alpha} \varphi(x u)
$$

thus,

$$
\left(\phi(x)-x \phi^{\prime}(x)\right)-\left(\phi\left(x_{0}\right)-x_{0} \phi^{\prime}\left(x_{0}^{+}\right)\right) \lesssim \varphi(x) \int_{0}^{1} u^{\alpha-1} \mathrm{~d} u .
$$

We denote $c_{1}=\phi\left(x_{0}\right)-x_{0} \phi^{\prime}\left(x_{0}^{+}\right)$. Using the scaling property of $\varphi$, we conclude that

$$
c_{1}=\frac{c_{1}}{\varphi\left(x_{0}\right)} \cdot \varphi\left(x_{0}\right) \leq \frac{c_{1}}{\varphi\left(x_{0}\right)} c^{-1}\left(\frac{x_{0}}{x}\right)^{\alpha} \varphi(x) \leq \frac{c_{1} c^{-1}}{\varphi\left(x_{0}\right)} \cdot \varphi(x),
$$

provided that $x>x_{0}$, which proves (4.12) if $x_{0}>0$. For $x_{0}=0$ it is enough to observe that (2.9) implies that $\lim _{x \rightarrow 0^{+}} x \phi^{\prime}(x)=0$, and the claim follows.

Proposition 4.6. Suppose that $-\phi^{\prime \prime} \in \operatorname{WLSC}\left(\alpha-2, c, x_{0}\right) \cap \operatorname{WUSC}\left(\beta-2, C, x_{0}\right)$ for some $c \in(0,1], C \geq 1, x_{0} \geq 0$ and $0<\alpha \leq \beta<1$. Assume also that $b=0$. Then for all $x>x_{0}$,

$$
\varphi^{*}(x) \approx \phi(x),
$$

and for all $r>\varphi\left(x_{0}\right)$,

$$
\varphi^{-1}(r) \approx \phi^{-1}(r)
$$

Furthermore, there is $c^{\prime} \in(0,1]$ such that for all $\lambda \geq 1$ and $r>\varphi\left(x_{0}\right)$,

$$
\varphi^{-1}(\lambda r) \geq c^{\prime} \lambda^{1 / \beta} \varphi^{-1}(r) .
$$

Proof. Let us observe that, by (2.9), Proposition 2.3 and Proposition 2.4, there is $c_{1} \in$ $(0,1]$ such that for all $x>x_{0}$,

$$
2 \phi(x) \geq \varphi(x) \geq c_{1} \phi(x) .
$$

Now the proof of the lemma is similar to the proof of Proposition 4.3 and is therefore omitted. 


\subsection{Estimates from above}

In this section we show the upper estimates on $p(t, \cdot)$. Before embarking on the proof, let us introduce some notation. Given a set $B \subset \mathbb{R}$, we define

$$
\delta(B)=\inf \{|x|: x \in B\}
$$

and

$$
\operatorname{diam}(B)=\sup \{|x-y|: x, y \in B\}
$$

Let

$$
b_{r}=b+\int_{(0, r)} s \nu(\mathrm{d} s), \quad r>0 .
$$

In view of (2.2), the above definition of $b_{r}$ is in line with the usual one (see, e.g., [32, formula (4)] or $[23$, formula (1.2)]). Let us define $\zeta:[0, \infty) \rightarrow[0, \infty]$,

$$
\zeta(s)= \begin{cases}\infty & \text { if } s=0, \\ \varphi^{*}(1 / s) & \text { if } 0<s \leq x_{0}^{-1} \\ A \phi(1 / s) & \text { if } x_{0}^{-1}<s,\end{cases}
$$

where $A=\varphi^{*}\left(x_{0}\right) / \phi\left(x_{0}\right) \in(0,2]$.

Theorem 4.7. Let $\mathbf{T}$ be a subordinator with the Lévy-Khintchine exponent $\psi$ and the Laplace exponent $\phi$. Suppose that $-\phi^{\prime \prime} \in \mathrm{WLSC}\left(\alpha-2, c, x_{0}\right)$ for some $c \in(0,1], x_{0} \geq 0$ and $\alpha>0$. Then the probability distribution of $T_{t}$ has a density $p(t, \cdot)$. Moreover, there is $C>0$ such that for all $t \in\left(0,1 / \varphi\left(x_{0}\right)\right)$ and $x \in \mathbb{R}$,

$$
p\left(t, x+t b_{1 / \psi^{-1}(1 / t)}\right) \leq C \varphi^{-1}(1 / t) \cdot \min \{1, t \zeta(|x|)\} .
$$

In particular, for all $t \in\left(0,1 / \varphi\left(x_{0}\right)\right)$ and $x \geq 2 e t \phi^{\prime}\left(\psi^{-1}(1 / t)\right)$,

$$
p(t, x+t b) \leq C \varphi^{-1}(1 / t) \cdot \min \{1, t \zeta(x)\} .
$$

Proof. Without loss of generality, we can assume $b=0$. Indeed, otherwise it is enough to consider a shifted process $\widetilde{T}_{t}=T_{t}-t b$. Next, let us observe that for any Borel set $B \subset \mathbb{R}$, we have

$$
\begin{aligned}
\nu(B) & \lesssim \int_{(\delta(B), \infty)}\left(1-e^{-s / \delta(B)}\right) \nu(\mathrm{d} s) \\
& \leq \phi(1 / \delta(B)) .
\end{aligned}
$$

Furthermore, for $\delta(B)<1 / x_{0}$, by Proposition 4.1 and Remark 3.2,

$$
\begin{aligned}
\nu(B) & \leq h(\delta(B)) \\
& \lesssim \varphi^{*}(1 / \delta(B)) .
\end{aligned}
$$

Thus, $\nu(B) \lesssim \zeta(\delta(B))$. We claim that $\zeta$ has doubling property on $(0, \infty)$. Indeed, since $-\phi^{\prime \prime}$ is nonincreasing function with the weak lower scaling property, it has doubling property 
on $\left(x_{0}, \infty\right)$; thus, for $0<s<x_{0}^{-1}$,

$$
\zeta\left(\frac{1}{2} s\right) \approx 4 s^{-2}\left(-\phi^{\prime \prime}(2 / s)\right) \lesssim s^{-2}\left(-\phi^{\prime \prime}(1 / s)\right) \lesssim \zeta(s)
$$

This completes the argument in the case $x_{0}=0$. If $x_{0}>0$, then by (2.10), for $s>2 x_{0}^{-1}$ we have

$$
\zeta\left(\frac{1}{2} s\right)=A \phi(2 / s) \leq 2 A \phi(1 / s) \leq 2 \zeta(s)
$$

Lastly, the function

$$
\left[\frac{1}{2} x_{0}, x_{0}\right] \ni x \mapsto \frac{\varphi^{*}(2 x)}{\phi(x)}
$$

is continuous and thus it is bounded.

Next, for $s>0$ and $x \in \mathbb{R}$,

$$
s \vee|x|-\frac{1}{2}|x| \geq \frac{1}{2} s ;
$$

thus, by motonicity and the doubling property of $\zeta$, we get

$$
\zeta\left(s \vee|x|-\frac{1}{2}|x|\right) \lesssim \zeta(s)
$$

Hence, by (2.4) and (4.2), for $r>0$,

$$
\begin{aligned}
\int_{(r, \infty)} \zeta\left(s \vee x-\frac{1}{2} x\right) \nu(\mathrm{d} x) & \lesssim \zeta(s) h(r) \\
& \lesssim \zeta(s) \psi^{*}(1 / r)
\end{aligned}
$$

Since $\psi^{*}$ has the weak lower scaling property and satisfies (4.3), by [23, Proposition 3.4] together with Proposition 4.2, there are $C>0$ and $t_{1} \in(0, \infty]$ such that for all $t \in\left(0, t_{1}\right)$,

$$
\int_{\mathbb{R}} e^{-t \Re \psi(\xi)}|\xi| \mathrm{d} \xi \leq C\left(\psi^{-1}(1 / t)\right)^{2} .
$$

If $x_{0}=0$, then $t_{1}=\infty$. If $t_{1}<1 / \varphi\left(x_{0}\right)$, we can expand the above estimate for $t_{1} \leq t<$ $1 / \varphi\left(x_{0}\right)$ using positivity of the right-hand side and monotonicity of the left-hand side.

In view of (4.19), (4.20) and (4.21), by [32, Theorem 1] with $\gamma=0$, there are $C_{1}, C_{2}, C_{3}>$ 0 such that for all $t \in\left(0,1 / \varphi\left(x_{0}\right)\right)$ and $x \in \mathbb{R}$,

$$
\begin{aligned}
& p\left(t, x+t b_{1 / \psi^{-1}(1 / t)}\right) \\
& \quad \leq C_{1} \psi^{-1}(1 / t) \cdot \min \left\{1, t \zeta\left(\frac{1}{4}|x|\right)+\exp \left\{-C_{2}|x| \psi^{-1}(1 / t) \log \left(1+C_{3}|x| \psi^{-1}(1 / t)\right)\right\}\right\} .
\end{aligned}
$$

Let us consider $x>0$ and $t \in\left(0,1 / \varphi\left(x_{0}\right)\right)$ such that $t \zeta(x) \leq 1$. We claim that

$$
\exp \left\{-C_{2} x \psi^{-1}(1 / t) \log \left(1+C_{3} x \psi^{-1}(1 / t)\right)\right\} \lesssim t \zeta(x)
$$

First suppose that $x>x_{0}^{-1}$. Let us observe that the function

$$
[0, \infty) \ni u \mapsto u \exp \left\{-C_{2} u \log \left(1+C_{3} u\right)\right\}
$$


is bounded. Therefore,

$$
\exp \left\{-C_{2} x \psi^{-1}(1 / t) \log \left(1+C_{3} x \psi^{-1}(1 / t)\right)\right\} \lesssim \frac{1}{x \psi^{-1}(1 / t)} .
$$

Since $x \phi^{-1}(1 / t) \geq 1$, by (2.10), we have

$$
t \phi(1 / x)=\frac{\phi(1 / x)}{\phi\left(x \phi^{-1}(1 / t) \cdot 1 / x\right)} \geq \frac{1}{x \phi^{-1}(1 / t)} .
$$

Next, in light of (2.9), for all $y>0$,

$$
\frac{1}{2} \varphi^{*}(y) \leq \phi(y)
$$

hence, by the monotonicity of $\phi^{-1}$,

$$
\begin{aligned}
\phi^{-1}(1 / t) & =\phi^{-1}\left(\frac{1}{2} \varphi^{*}\left(\varphi^{-1}(2 / t)\right)\right) \\
& \leq \phi^{-1}\left(\phi\left(\varphi^{-1}(2 / t)\right)\right) \\
& =\varphi^{-1}(2 / t) \\
& \leq C \psi^{-1}(1 / t),
\end{aligned}
$$

where in the last step we have used Proposition 4.3. Putting (4.23), (4.24) and (4.25) together, we obtain (4.22) as claimed.

Now let $0<x \leq x_{0}^{-1}$. Observe that the function

$$
[0, \infty) \ni u \mapsto u^{2} \exp \left\{-C_{2} u \log \left(1+C_{3} u\right)\right\}
$$

is also bounded. Hence,

$$
\exp \left\{-C_{2} x \psi^{-1}(1 / t) \log \left(1+C_{3} x \psi^{-1}(1 / t)\right)\right\} \lesssim \frac{1}{\left(x \psi^{-1}(1 / t)\right)^{2}} .
$$

Since $x \varphi^{-1}(1 / t) \geq 1$, using (4.5) we get

$$
t \varphi^{*}(1 / x)=\frac{\varphi^{*}(1 / x)}{\varphi^{*}\left(x \varphi^{-1}(1 / t) \cdot 1 / x\right)} \geq \frac{1}{\left(x \varphi^{-1}(1 / t)\right)^{2}} .
$$

Hence, putting together (4.26) and (4.27) and invoking Proposition 4.2, we again obtain (4.22).

Finally, using doubling property of $\zeta$ we get

$$
\zeta\left(\frac{1}{4} x\right) \lesssim \zeta(x)
$$

thus, another application of Proposition 4.3 leads to (4.17).

For the proof of (4.18), we observe that

$$
\phi^{\prime}(\lambda)=\int_{(0, \infty)} x e^{-\lambda x} \nu(\mathrm{d} x) \geq e^{-1} \int_{(0,1 / \lambda)} x \nu(\mathrm{d} x) .
$$


Thus,

$$
b_{1 / \psi^{-1}(1 / t)}=\int_{\left(0,1 / \psi^{-1}(1 / t)\right)} x \nu(\mathrm{d} x) \leq e \phi^{\prime}\left(\psi^{-1}(1 / t)\right) .
$$

Hence, by monotonicity and the doubling property of $\zeta$, for $x>2 e t \phi^{\prime}\left(\psi^{-1}(1 / t)\right)$, we obtain

$$
\zeta\left(x-t b_{1 / \psi^{-1}(1 / t)}\right) \leq \zeta\left(\frac{x}{2}\right) \lesssim \zeta(x)
$$

and the theorem follows.

Now we define $\eta:[0, \infty) \rightarrow[0, \infty]$,

$$
\eta(s)=s^{-1} \zeta(s)= \begin{cases}\infty & \text { if } s=0, \\ s^{-1} \varphi^{*}(1 / s) & \text { if } 0<s \leq x_{0}^{-1} \\ A s^{-1} \phi(1 / s) & \text { if } x_{0}^{-1}<s,\end{cases}
$$

where $A=\varphi^{*}\left(x_{0}\right) / \phi\left(x_{0}\right) \in(0,2]$. Notice that, by $(2.9)$, if $2 t \zeta(|x|) \leq 1$, then $t \varphi^{*}(1 /|x|) \leq 1$, and so

$$
\begin{aligned}
\eta(|x|) & =|x|^{-1} \zeta(|x|) \\
& \leq \varphi^{-1}(1 / t) \zeta(|x|)
\end{aligned}
$$

Therefore,

$$
\min \left\{\varphi^{-1}(1 / t), \operatorname{t\eta }(|x|)\right\} \leq 4 \varphi^{-1}(1 / t) \cdot \min \{1, t \zeta(|x|)\} .
$$

Theorem 4.8. Let $\mathbf{T}$ be a subordinator with the Lévy-Khintchine exponent $\psi$ and the Laplace exponent $\phi$. Suppose that $-\phi^{\prime \prime} \in \mathrm{WLSC}\left(\alpha-2, c, x_{0}\right)$ for some $c \in(0,1], x_{0} \geq 0$ and $\alpha>0$. We also assume that the Lévy measure $\nu$ has an almost monotone density $\nu(x)$. Then the probability distribution of $T_{t}$ has a density $p(t, \cdot)$. Moreover, there is $C>0$ such that for all $t \in\left(0,1 / \varphi\left(x_{0}\right)\right)$ and $x \in \mathbb{R}$,

$$
p\left(t, x+t b_{1 / \psi^{-1}(1 / t)}\right) \leq C \min \left\{\varphi^{-1}(1 / t), t \eta(|x|)\right\} .
$$

In particular, for all $t \in\left(0,1 / \varphi\left(x_{0}\right)\right)$ and $x \geq 2 e t \phi^{\prime}\left(\psi^{-1}(1 / t)\right)$,

$$
p(t, x+t b) \leq C \min \left\{\varphi^{-1}(1 / t), t \eta(x)\right\} .
$$

Proof. Without loss of generality, we can assume $b=0$. Let us observe that for any $\lambda>0$,

$$
\phi(\lambda) \geq \int_{0}^{1 / \lambda}\left(1-e^{-\lambda s}\right) \nu(s) \mathrm{d} s \gtrsim \nu(1 / \lambda) \lambda^{-1}
$$

and

$$
-\phi^{\prime \prime}(\lambda) \geq \int_{0}^{1 / \lambda} s^{2} e^{-\lambda s} \nu(s) \mathrm{d} s \gtrsim \nu(1 / \lambda) \lambda^{-3} .
$$


Hence,

$$
\nu(x) \lesssim \eta(x) \quad \text { for all } x>0 .
$$

Since $\eta$ is nonincreasing, for any Borel subset $B \subset \mathbb{R}$,

$$
\nu(B) \lesssim \int_{B \cap(0, \infty)} \eta(x) \mathrm{d} x \lesssim \eta(\delta(B)) \operatorname{diam}(B) .
$$

Arguing as in the proof of Theorem 4.7, we conclude that $\eta$ has a doubling property on $(0, \infty)$. Using that and monotonicity of $\eta$, for $s>0$ and $x \in \mathbb{R}$,

$$
\eta\left(s \vee x-\frac{1}{2} x\right) \leq \eta\left(\frac{1}{2} s\right) \lesssim \eta(s) .
$$

Therefore, by (4.2), for $r>0$,

$$
\int_{r}^{\infty} \eta\left(s \vee x-\frac{1}{2} x\right) \nu(x) \mathrm{d} x \lesssim \eta(s) \psi^{*}(1 / r) .
$$

Since $\psi^{*}$ has the weak lower scaling property and satisfies (4.3), by [23, Theorem 3.1] and Proposition 4.2, there are $C>0$ and $t_{1} \in(0, \infty]$ such that for all $t \in\left(0, t_{1}\right)$,

$$
\int_{\mathbb{R}} e^{-t \Re \psi(\xi)} \mathrm{d} \xi \leq C \psi^{-1}(1 / t) .
$$

If $x_{0}=0$, then $t_{1}=\infty$. If $t_{1}<48 / \varphi\left(x_{0}\right)$, we can expand the above estimate for $t_{1} \leq t<$ $48 / \varphi\left(x_{0}\right)$ using positivity of the right-hand side and monotonicity of the left-hand side.

In view of (4.31), (4.32) and (4.33), by [22, Theorem 2.1], there is $C>0$ such that for all $t \in\left(0,1 / \varphi\left(x_{0}\right)\right)$ and $x \in \mathbb{R}$,

$$
p\left(t, x+t b_{1 / \psi^{-1}(1 / t)}\right) \leq C \psi^{-1}(1 / t) \cdot \min \left\{1, t\left(\psi^{-1}(1 / t)\right)^{-1} \eta(|x|)+\left(1+|x| \psi^{-1}(1 / t)\right)^{-3}\right\} .
$$

We claim that

$$
\frac{\psi^{-1}(1 / t)}{\left(1+|x| \psi^{-1}(1 / t)\right)^{3}} \lesssim t \eta(|x|)
$$

whenever $\operatorname{t\eta }(|x|) \leq \frac{A}{2} \varphi^{-1}(1 / t)$.

First, let us show that for any $\epsilon \in(0,1]$, the condition $t \eta(|x|) \leq \frac{A \epsilon}{2} \varphi^{-1}(1 / t)$ implies that

$$
t \varphi^{*}\left(\frac{1}{|x|}\right) \leq \epsilon|x| \varphi^{-1}\left(\frac{1}{t}\right) .
$$

Indeed, by (2.9), we have $|x| \eta(|x|) \geq \frac{A}{2} \varphi^{*}(1 /|x|)$; thus,

$$
\epsilon|x| \varphi^{-1}\left(\frac{1}{t}\right) \geq \frac{2}{A} t|x| \eta(|x|) \geq t \varphi^{*}\left(\frac{1}{|x|}\right) .
$$

Notice also that $\epsilon^{1 / 3}|x| \varphi^{-1}(1 / t) \geq 1$ since otherwise, by (4.5),

$$
1<t \varphi^{*}\left(\frac{1}{\epsilon^{1 / 3}|x|}\right)<\frac{1}{\epsilon^{2 / 3}} t \varphi^{*}\left(\frac{1}{|x|}\right),
$$


which entails that $\epsilon^{2 / 3}<t \varphi^{*}(1 /|x|)$; that is, $\epsilon^{1 / 3}|x| \varphi^{-1}(1 / t)<\epsilon^{-2 / 3} t \varphi^{*}(1 /|x|)$, contrary to $(4.35)$.

To show (4.34), let us suppose that $t \eta(|x|) \leq \frac{A}{2} \varphi^{-1}(1 / t)$; thus, $|x| \varphi^{-1}(1 / t) \geq 1$. By (4.5), we have

$$
t \varphi^{*}(1 /|x|)=\frac{\varphi^{*}(1 /|x|)}{\varphi^{*}\left(|x| \varphi^{-1}(1 / t) \cdot 1 /|x|\right)} \geq \frac{1}{\left(|x| \varphi^{-1}(1 / t)\right)^{2}},
$$

which, by Proposition 4.3, gives

$$
t|x| \eta(|x|) \geq \frac{A}{2} t \varphi^{*}(1 /|x|) \gtrsim \frac{|x| \psi^{-1}(1 / t)}{\left(1+|x| \psi^{-1}(1 / t)\right)^{3}},
$$

proving (4.34), and (4.28) follows. The inequality (4.29) holds by the same argument as in the proof of Theorem 4.7 .

Remark 4.9. In statements of Theorems 4.7 and 4.8 , we can replace $b_{1 / \psi^{-1}(1 / t)}$ by $b_{1 / \varphi^{-1}(1 / t)}$. Indeed, let us observe that if $0<r_{1} \leq r_{2}<1 / x_{0}$, then

$$
\begin{aligned}
\left|b_{r_{1}}-b_{r_{2}}\right| & \leq \int_{\left(r_{1}, r_{2}\right]} s \nu(\mathrm{d} s) \\
& \leq r_{1}^{-1} r_{2}^{2} h\left(r_{2}\right) \\
& \lesssim r_{1}^{-1} r_{2}^{2} \psi^{*}\left(1 / r_{2}\right),
\end{aligned}
$$

where in the last estimate we have used (4.2). Hence, by (4.9), we get

$$
\left|b_{r_{1}}-b_{r_{2}}\right| \lesssim r_{1}^{-1} r_{2}^{2} \varphi^{*}\left(1 / r_{2}\right) .
$$

Therefore, by (4.36), (4.37) and Proposition 4.3, there is $C \geq 1$ such that

$$
\left|b_{1 / \psi^{-1}(1 / t)}-b_{1 / \varphi^{-1}(1 / t)}\right| \leq C \frac{1}{t \varphi^{-1}(1 / t)},
$$

provided that $0<t<1 / \varphi\left(x_{0}\right)$. Now, let us suppose that $8 C^{2} t \zeta(|x|) \leq 1$. Then, by $(2.10)$ and (4.5),

$$
\frac{1}{t} \geq 8 C^{2} \zeta(|x|) \geq 4 C^{2} \varphi^{*}\left(\frac{1}{|x|}\right) \geq \varphi^{*}\left(\frac{2 C}{|x|}\right)
$$

that is,

$$
|x| \geq \frac{2 C}{\varphi^{-1}(1 / t)}
$$

Hence, by (4.38),

$$
\left|x+t\left(b_{1 / \psi^{-1}(1 / t)}-b_{1 / \varphi^{-1}(1 / t)}\right)\right| \geq|x|-\frac{C}{\varphi^{-1}(1 / t)} \geq \frac{|x|}{2},
$$

which, together with monotonicity and the doubling property of $\zeta$, gives

$$
\zeta\left(\left|x+t\left(b_{1 / \psi^{-1}(1 / t)}-b_{1 / \varphi^{-1}(1 / t)}\right)\right|\right) \lesssim \zeta(|x|) .
$$


Similarly, if $t \eta(|x|) \leq \frac{A \epsilon}{2} \varphi^{-1}(1 / t)$, then

$$
|x| \varphi^{-1}(1 / t) \geq \epsilon^{-1 / 3}
$$

thus, by taking $\epsilon=(2 C)^{-3}$, we obtain (4.39). Hence, by monotonicity and the doubling property of $\eta$, we again obtain

$$
\eta\left(\left|x+t\left(b_{1 / \psi^{-1}(1 / t)}-b_{1 / \varphi^{-1}(1 / t)}\right)\right|\right) \lesssim \eta(|x|) .
$$

\subsection{Estimates from below}

In this section we develop estimates from below on the density $p(t, \cdot)$. The main result is Theorem 4.11. Its proof is inspired by the ideas from [42], see also [23]. Thanks to Theorem 3.3, we can generalise results obtained in [42] to the case when $-\phi^{\prime \prime}$ satisfies the weak lower scaling of index $\alpha-2$ for $\alpha>0$ together with a certain additional condition. We use the following variant of the celebrated Pruitt's result [45, Section 3] adapted to subordinators.

Proposition 4.10. Let $\mathbf{T}$ be a subordinator with the Lévy-Khintchine exponent

$$
\psi(\xi)=-i \xi b-\int_{(0, \infty)}\left(e^{i \xi x}-1\right) \nu(d x) .
$$

Then there is an absolute constant $c>0$ such that for all $\lambda>0$ and $t>0$,

$$
\mathbb{P}\left(\sup _{0 \leq s \leq t}\left|T_{s}-s b_{\lambda}\right| \geq \lambda\right) \leq \operatorname{cth}(\lambda) .
$$

Proof. We are going to apply the estimates [45, (3.2)]. To do so, we need to express the Lévy-Khintchine exponent of $T_{s}-s b_{\lambda}$ in the form used in [45, Section 3], namely,

$$
\begin{aligned}
\tilde{\psi}(\xi) & =\psi(\xi)+i \xi b_{\lambda} \\
& =-i \xi\left(b-b_{\lambda}+\int_{(0, \infty)} \frac{y}{1+|y|^{2}} \nu(\mathrm{d} y)\right)-\int_{(0, \infty)}\left(e^{i \xi y}-1-\frac{i y \xi}{1+|y|^{2}}\right) \nu(\mathrm{d} y) .
\end{aligned}
$$

Since

$$
\int_{(0, \lambda]} \frac{y|y|^{2}}{1+|y|^{2}} \nu(\mathrm{d} y)-\int_{(\lambda, \infty)} \frac{y}{1+|y|^{2}} \nu(\mathrm{d} y)=\int_{(0, \lambda]} y \nu(\mathrm{d} y)-\int_{(0, \infty)} \frac{y}{1+|y|^{2}} \nu(\mathrm{d} y),
$$

we have

$$
\begin{aligned}
M(\lambda) & =\frac{1}{\lambda}\left|b-b_{\lambda}+\int_{(0, \infty)} \frac{y}{1+|y|^{2}} \nu(\mathrm{d} y)+\int_{(0, \lambda]} \frac{y|y|^{2}}{1+|y|^{2}} \nu(\mathrm{d} y)-\int_{(\lambda, \infty)} \frac{y}{1+|y|^{2}} \nu(\mathrm{d} y)\right| \\
& =\frac{1}{\lambda}\left|b-b_{\lambda}+\int_{(0, \lambda]} y \nu(\mathrm{d} y)\right|=0 .
\end{aligned}
$$

Hence, by [45, (3.2)],

$$
\mathbb{P}\left(\sup _{0 \leq s \leq t}\left|T_{s}-s b_{\lambda}\right| \geq \lambda\right) \leq \operatorname{csh}(\lambda)
$$

as desired. 
Theorem 4.11. Let $\mathbf{T}$ be a subordinator with the Laplace exponent $\phi$. Suppose that $-\phi^{\prime \prime} \in \mathrm{WLSC}\left(\alpha-2, c, x_{0}\right)$ for some $c \in(0,1], x_{0} \geq 0$ and $\alpha>0$, and assume that one of the following conditions holds true:

(i) $-\phi^{\prime \prime} \in \operatorname{WUSC}\left(\beta-2, C, x_{0}\right)$ for some $C \geq 1$ and $\alpha \leq \beta<1$, or

(ii) $-\phi^{\prime \prime}$ is a function regularly varying at infinity with index -1 . If $x_{0}=0$ we also assume that $-\phi^{\prime \prime}$ is regularly varying at zero with index -1 .

Then there is $M_{0}>1$ such that for each $M \geq M_{0}$ there exists $\rho_{0}>0$, so that for all $0<\rho_{1}<\rho_{0}, 0<\rho_{2}$ there is $C>0$ such that for all $t \in\left(0,1 / \varphi\left(x_{0}\right)\right)$ and all $x>0$ satisfying

$$
-\frac{\rho_{1}}{\varphi^{-1}(1 / t)} \leq x-t \phi^{\prime}\left(\varphi^{-1}(M / t)\right) \leq \frac{\rho_{2}}{\varphi^{-1}(1 / t)}
$$

we have

$$
p(t, x) \geq C \varphi^{-1}(1 / t) .
$$

Remark 4.12. From the proof of Theorem 4.11 it stems that if $x_{0}=0$, one can obtain the same statement under the condition that $-\phi^{\prime \prime}$ is $(-1)$-regular at infinity and satisfies upper scaling at 0 with $\alpha \leq \beta<1$. Alternatively, one can assume that $-\phi^{\prime \prime}$ satisfies upper scaling at infinity with $\alpha \leq \beta<1$ and varies regularly at zero with index -1 . The same remark applies to Proposition 4.14.

Proof. First let us observe that it is enough to prove that (4.40) holds true for all $t \in\left(0,1 / \varphi\left(x_{0}\right)\right)$ and all $x>0$ satisfying

$$
-\frac{\rho_{1}}{\varphi^{-1}(M / t)} \leq x-t \phi^{\prime}\left(\varphi^{-1}(M / t)\right) \leq \frac{\rho_{2}}{\varphi^{-1}(M / t)} .
$$

Indeed, since $\varphi^{-1}$ is nondecreasing and has upper scaling property (see Proposition 4.3), it has a doubling property. Hence, the lemma will follow immediately with possibly modified $\rho_{0}$.

Without loss of generality, we can assume that $b=0$. Let $\lambda>0$, whose value will be specified later. We decompose the Lévy measure $\nu(\mathrm{d} x)$ as follows: Let $\nu_{1}(\mathrm{~d} x)$ be the restriction of $\frac{1}{2} \nu(\mathrm{d} x)$ to the interval $(0, \lambda]$ and

$$
\nu_{2}(\mathrm{~d} x)=\nu(\mathrm{d} x)-\nu_{1}(\mathrm{~d} x) .
$$

We set

$$
\phi_{1}(u)=\int_{(0, \infty)}\left(1-e^{-u s}\right) \nu_{1}(\mathrm{~d} s), \quad \phi_{2}(u)=\int_{(0, \infty)}\left(1-e^{-u s}\right) \nu_{2}(\mathrm{~d} s) .
$$

Let us denote by $\mathbf{T}^{(j)}$ the subordinator having the Laplace exponent $\phi_{j}$, for $j \in\{1,2\}$. Let $\psi_{j}(\xi)=\phi_{j}(-i \xi)$. Notice that $\frac{1}{2} \nu \leq \nu_{2} \leq \nu ;$ thus,

$$
\frac{1}{2} \phi \leq \phi_{2} \leq \phi
$$

and for every $n \in \mathbb{N}$,

$$
\frac{1}{2}(-1)^{n+1} \phi^{(n)} \leq(-1)^{n+1} \phi_{2}^{(n)} \leq(-1)^{n+1} \phi^{(n)} .
$$


Therefore, for all $u>0$,

$$
\frac{1}{2} \varphi(u) \leq \varphi_{2}(u) \leq \varphi(u)
$$

Next, by Theorem 3.3, the random variables $T_{t}^{(2)}$ and $T_{t}$ are absolutely continuous. Let us denote by $p^{(2)}(t, \cdot)$ and $p(t, \cdot)$ the densities of $T_{t}^{(2)}$ and $T_{t}$, respectively.

Let $M \geq 2 M_{0}+1$, where $M_{0}$ is determined in Corollary 3.5 for the process $\mathbf{T}^{(2)}$. For $0<t<1 / \varphi\left(x_{0}\right)$, we set

$$
x_{t}=t \phi_{2}^{\prime}\left(\varphi^{-1}(M / t)\right) .
$$

Since $\varphi^{-1}(M / t)>x_{0}$, we have

$$
\frac{x_{t}}{t}=\phi_{2}^{\prime}\left(\varphi^{-1}(M / t)\right) \leq \phi_{2}^{\prime}\left(x_{0}\right)
$$

Let

$$
w_{2}=\left(\phi_{2}^{\prime}\right)^{-1}\left(x_{t} / t\right)=\varphi^{-1}(M / t)
$$

Then, by (4.42) we get

$$
\varphi_{2}\left(w_{2}\right) \geq \frac{1}{2} \varphi\left(\varphi^{-1}(M / t)\right)=\frac{M}{2 t} \geq \frac{M_{0}}{t} .
$$

Moreover, by Corollary 4.5 together with (4.42) we get

$$
t\left(\phi_{2}\left(w_{2}\right)-w_{2} \phi_{2}^{\prime}\left(w_{2}\right)\right) \lesssim t \varphi_{2}\left(w_{2}\right) \lesssim 1
$$

Hence, by Corollary 3.5,

$$
p^{(2)}\left(t, x_{t}\right) \gtrsim \frac{1}{\sqrt{t\left(-\phi_{2}^{\prime \prime}\right)\left(w_{2}\right)}} .
$$

Notice that, by (4.41) and Remark 3.4, the implied constant in (4.43) is independent of $t$ and $\lambda$. Since

$$
\left(-\phi_{2}^{\prime \prime}\right)\left(w_{2}\right) \leq\left(-\phi^{\prime \prime}\right)\left(\varphi^{-1}(M / t)\right)=\frac{M}{t\left(\varphi^{-1}(M / t)\right)^{2}},
$$

by (4.43) and monotonicity of $\varphi^{-1}$, we get

$$
p^{(2)}\left(t, x_{t}\right) \geq C_{1} \varphi^{-1}(1 / t)
$$

for some constant $C_{1}>0$.

Next, by the Fourier inversion formula

$$
\begin{aligned}
\sup _{x \in \mathbb{R}}\left|\partial_{x} p^{(2)}(t, x)\right| & \lesssim \int_{\mathbb{R}} e^{-t \Re \psi_{2}(\xi)}|\xi| \mathrm{d} \xi \\
& \lesssim \int_{\mathbb{R}} e^{-\frac{t}{2} \Re \psi(\xi)}|\xi| \mathrm{d} \xi
\end{aligned}
$$


thus, by [23, Proposition 3.4] and Propositions 4.2 and 4.3 we see that there is $C_{2}>0$ such that for all $t \in\left(0,1 / \varphi\left(x_{0}\right)\right)$,

$$
\sup _{x \in \mathbb{R}}\left|\partial_{x} p^{(2)}(t, x)\right| \leq C_{2}\left(\varphi^{-1}(1 / t)\right)^{2} .
$$

By the mean value theorem, for $y \in \mathbb{R}$, we get

$$
\left|p^{(2)}\left(t, y+x_{t}\right)-p^{(2)}\left(t, x_{t}\right)\right| \leq C_{2}|y|\left(\varphi^{-1}(1 / t)\right)^{2} .
$$

Hence, for $y \in \mathbb{R}$ satisfying

$$
|y| \leq \frac{C_{1}}{2 C_{2} \varphi^{-1}(1 / t)},
$$

by (4.44), we get

$$
\begin{aligned}
p^{(2)}\left(t, y+x_{t}\right) & \geq p^{(2)}\left(t, x_{t}\right)-C_{2}|y|\left(\varphi^{-1}(1 / t)\right)^{2} \\
& \geq \frac{C_{1}}{2} \varphi^{-1}(1 / t) .
\end{aligned}
$$

Therefore,

$$
\begin{aligned}
p(t, x) & =\int_{\mathbb{R}} p^{(2)}(t, x-y) \mathbb{P}\left(T_{t}^{(1)} \in \mathrm{d} y\right) \\
& \geq \frac{C_{1}}{2} \varphi^{-1}(1 / t) \cdot \mathbb{P}\left(\left|x-x_{t}-T_{t}^{(1)}\right| \leq \frac{C_{0}}{\varphi^{-1}(1 / t)}\right) \\
& \geq \frac{C_{1}}{2} \varphi^{-1}(1 / t) \cdot \mathbb{P}\left(\left|x-x_{t}-T_{t}^{(1)}\right| \leq \frac{C_{0}}{\varphi^{-1}(M / t)}\right) \\
& =\frac{C_{1}}{2} \varphi^{-1}(1 / t) \cdot \mathbb{P}\left(\left|x-\tilde{x}_{t}-\left(\frac{1}{2} t b_{\lambda}-\left(\tilde{x}_{t}-x_{t}\right)\right)-\left(T_{t}^{(1)}-\frac{1}{2} t b_{\lambda}\right)\right| \leq \frac{C_{0}}{\varphi^{-1}(M / t)}\right),
\end{aligned}
$$

where we have set $C_{0}=C_{1}\left(2 C_{2}\right)^{-1}$ and

$$
\tilde{x}_{t}=t \phi^{\prime}\left(\varphi^{-1}(M / t)\right) \text {. }
$$

Let $\rho_{0}=\frac{1}{2} C_{0}$ and

$$
\lambda=\frac{1}{\varphi^{-1}(M / t)} .
$$

We have

$$
\begin{aligned}
\frac{1}{2} t b_{\lambda}-\left(\tilde{x}_{t}-x_{t}\right) & =\frac{1}{2} t b_{\lambda}-t \phi_{1}^{\prime}(1 / \lambda) \\
& =\frac{t}{2} \int_{(0, \lambda]} s\left(1-e^{-s / \lambda}\right) \nu(\mathrm{d} s) .
\end{aligned}
$$

Thus, $\frac{1}{2} t b_{\lambda}-\left(\tilde{x}_{t}-x_{t}\right)$ is nonnegative, and in view of (4.2) and (4.45),

$$
\begin{aligned}
\frac{1}{2} t b_{\lambda}-\left(\tilde{x}_{t}-x_{t}\right) & \leq C_{3} t \lambda \varphi(1 / \lambda) \\
& =\frac{C_{3} M}{\varphi^{-1}(M / t)}
\end{aligned}
$$


for some constant $C_{3}>0$. Next, setting

$$
\rho(t)=\lambda^{-1}\left(\frac{1}{2} t b_{\lambda}-\left(\tilde{x}_{t}-x_{t}\right)\right)
$$

we get

$$
\begin{gathered}
\inf _{t \in\left(0,1 / \varphi\left(x_{0}\right)\right)}\left\{\mathbb{P}\left(\left|x-\tilde{x}_{t}-\lambda \rho(t)-\left(T_{t}^{(1)}-\frac{1}{2} t b_{\lambda}\right)\right| \leq C_{0} \lambda\right): x \geq 0,-\rho_{1} \lambda \leq x-\tilde{x}_{t} \leq \rho_{2} \lambda\right\} \\
\geq \inf _{t \in\left(0,1 / \varphi\left(x_{0}\right)\right)}\left\{\mathbb{P}\left(\left|y-\lambda^{-1}\left(T_{t}^{(1)}-\frac{1}{2} t b_{\lambda}\right)\right| \leq C_{0}\right):-\rho_{1}-\rho(t) \leq y \leq \rho_{2}\right\} .
\end{gathered}
$$

Hence, the problem is reduced to showing that the infimum above is positive. Let us consider a collection $\left\{Y_{t}: t \in\left(0,1 / \varphi\left(x_{0}\right)\right)\right\}$ of infinitely divisible nonnegative random variables $Y_{t}=\lambda^{-1}\left(T_{t}^{(1)}-\frac{1}{2} t b_{\lambda}\right)$. The Lévy measure corresponding to $Y_{t}$ is

$$
\mu_{t}(B)=t \nu_{1}(\lambda B)
$$

for any Borel subset $B \subset \mathbb{R}$. Since for each $R>1$,

$$
\begin{aligned}
b_{R \lambda}^{(1)} & =\int_{(0, R \lambda]} y \nu_{1}(\mathrm{~d} y) \\
& =\frac{1}{2} \int_{(0, \lambda]} y \nu(\mathrm{d} y)=\frac{1}{2} b_{\lambda},
\end{aligned}
$$

by Proposition 4.10,

$$
\begin{aligned}
\mathbb{P}\left(\left|Y_{t}\right| \geq R\right) & =\mathbb{P}\left(\left|T_{t}^{(1)}-\frac{1}{2} t b_{\lambda}\right| \geq R \lambda\right) \\
& \lesssim t \int_{(0, \infty)} \min \left\{1, R^{-2} \lambda^{-2} s^{2}\right\} \nu_{1}(\mathrm{~d} s)
\end{aligned}
$$

thus,

$$
\begin{aligned}
\mathbb{P}\left(\left|Y_{t}\right| \geq R\right) & \lesssim t \lambda^{-2} R^{-2} \int_{(0, \lambda]} s^{2} \nu(\mathrm{d} s) \\
& \lesssim t R^{-2} h(\lambda) \\
& \lesssim t R^{-2} \varphi(1 / \lambda),
\end{aligned}
$$

where in the last estimate we have used (4.2). Therefore, recalling (4.45), we conclude that the collection is tight. Next, let $\left(\left(Y_{t_{n}}, y_{n}\right): n \in \mathbb{N}\right)$ be a sequence realising the infimum in (4.47). By the Prokhorov theorem, we can assume that $\left(Y_{t_{n}}: n \in \mathbb{N}\right)$ is weakly convergent to the random variable $Y_{0}$. We note that $Y_{t_{n}}$ has the probability distribution supported in $\left[-\frac{1}{2} t_{n} \lambda_{n}^{-1} b_{\lambda_{n}}, \infty\right)$ where $\lambda_{n}$ is defined as $\lambda$ corresponding to $t_{n}$.

Suppose that $\left(t_{n}: n \in \mathbb{N}\right)$ contains a subsequence convergent to $t_{0}>0$. Then $Y_{0}=Y_{t_{0}}$ and the support of its probability distribution equals $\left[-\frac{1}{2} t_{0} \lambda_{0}^{-1} b_{\lambda_{0}}, \infty\right)$. Since $\rho\left(t_{0}\right) \leq$ $\frac{1}{2} t_{0} \lambda_{0}^{-1} b_{\lambda_{0}}$, we easily conclude that the infimum in (4.47) is positive.

Hence, it remains to investigate the case when $\left(t_{n}: n \in \mathbb{N}\right)$ has no positive accumulation points. If zero is the only accumulation point, then $\left(\lambda_{n}: n \in \mathbb{N}\right)$ has a subsequence 
convergent to zero. Otherwise, $\left(t_{n}\right)$ diverges to infinity; thus, $x_{0}=0$ and $\left(\lambda_{n}\right)$ contains a subsequence diverging to infinity. In view of (4.46), $\rho(t)$ is uniformly bounded in $t$. Thus, after taking a subsequence, we may and do assume that there exists a limit

$$
\tilde{\rho}=\lim _{n \rightarrow \infty} \rho\left(t_{n}\right) .
$$

By compactness we can also assume that $\left(y_{n}: n \in \mathbb{N}\right)$ converges to $y_{0} \in\left[-\rho_{1}-\tilde{\rho}, \rho_{2}\right]$. Consequently, to prove that the infimum in (4.47) is positive, it is sufficient to show that

$$
\mathbb{P}\left(\left|y_{0}-Y_{0}\right| \leq \frac{1}{2} C_{0}\right)>0 .
$$

Observe that (4.49) is trivially satisfied if the support of the probability distribution of $Y_{0}$ is the whole real line. Therefore, we can assume that $Y_{0}$ is purely non-Gaussian. In view of [47, Theorem 8.7], it is also infinitely divisible.

Given $w: \mathbb{R} \rightarrow \mathbb{R}$ a continuous function satisfying

$$
|w(x)-1| \leq C^{\prime}|x|, \quad \text { and } \quad|w(x)| \leq C^{\prime}|x|^{-1},
$$

we write the Lévy-Khintchine exponent of $Y_{t_{n}}$ in the form

$$
\psi_{n}(\xi)=-i \xi \gamma_{n}-\int_{(0, \infty)}\left(e^{i \xi s}-1-i \xi s w(s)\right) \mu_{t_{n}}(\mathrm{~d} s)
$$

where

$$
\gamma_{n}=\int_{(0, \infty)} s w(s) \mu_{t_{n}}(\mathrm{~d} s)-\frac{1}{2} \lambda_{n}^{-1} t_{n} b_{\lambda_{n}} .
$$

Since $\left(Y_{t_{n}}: n \in \mathbb{N}\right)$ converges weakly to $Y_{0}$, there are $\gamma_{0} \in \mathbb{R}$ and $\sigma$-finite measure $\mu_{0}$ on $(0, \infty)$ satisfying

$$
\int_{(0, \infty)} \min \left\{1, s^{2}\right\} \mu_{0}(\mathrm{~d} s)<\infty
$$

such that the Lévy-Khintchine exponent of $Y_{0}$ is

$$
\psi_{0}(\xi)=-i \xi \gamma_{0}-\int_{(0, \infty)}\left(e^{i \xi s}-1-i \xi s w(s)\right) \mu_{0}(\mathrm{~d} s),
$$

where

$$
\gamma_{0}=\lim _{n \rightarrow \infty} \gamma_{n}
$$

Moreover, for any bounded continuous function $f: \mathbb{R} \rightarrow \mathbb{R}$ vanishing in a neighbourhood of zero, we have

$$
\lim _{n \rightarrow \infty} \int_{(0, \infty)} f(s) \mu_{t_{n}}(\mathrm{~d} s)=\int_{(0, \infty)} f(s) \mu_{0}(\mathrm{~d} s) .
$$

Next, let us fix $w$ satisfying (4.50) which equals 1 on $[0,1]$. In view of (4.48) and the definition of $\nu_{1}$, the support of $\mu_{t_{n}}$ is contained in $[0,1]$. Hence, $\gamma_{n}=0$ for every $n \in \mathbb{N}$ and, consequently, $\gamma_{0}=0$. We also conclude that $\operatorname{supp} \mu_{0} \subset[0,1]$. 
At this stage we consider the cases (i) and (ii) separately. In (ii) we need to distinguish two possibilities: if $\left(t_{n}\right)$ tends toward zero, then $\left(\lambda_{n}\right)$ also approaches zero, and we impose that $-\phi^{\prime \prime}$ is a function regularly varying at infinity with index -1 ; otherwise, $\left(t_{n}\right)$ tends toward infinity as well as $\left(\lambda_{n}\right)$, and thus $x_{0}=0$, and we additionally assume that $-\phi^{\prime \prime}$ is a function regularly varying at zero with index -1 . For the sake of clarity of presentation, we restrict attention to the first possibility only. In the second one the reasoning is analogous. We show that the support of the probability distribution of $Y_{0}$ is the whole real line. By [47, Theorem 24.10], the latter can be deduced from

$$
\int_{(0, \infty)} \min \{1, s\} \mu_{0}(\mathrm{~d} s)=\infty
$$

Since supp $\mu_{0} \subset[0,1]$, for each $\epsilon \in(0,1)$ we can write

$$
\int_{(0, \infty)} \min \{1, s\} \mu_{0}(\mathrm{~d} s) \geq \int_{(\epsilon / 2,1]} s \mu_{0}(\mathrm{~d} s)
$$

thus, to conclude (4.53), it is enough to show that

$$
\int_{(\epsilon / 2,1]} s \mu_{0}(\mathrm{~d} s) \gtrsim \log \epsilon^{-1}
$$

For the proof, for any $\epsilon \in(0,1)$ we define the following bounded continuous function:

$$
f_{\epsilon}(s)= \begin{cases}0 & \text { if } s<\epsilon / 2 \\ 2 s-\epsilon, & \text { if } \epsilon / 2 \leq s<\epsilon \\ s & \text { if } \epsilon \leq s<1 \\ 1 & \text { if } s \geq 1\end{cases}
$$

We have, in view of (4.52),

$$
\int_{(\epsilon / 2,1]} s \mu_{0}(\mathrm{~d} s) \geq \int_{(0,1]} f_{\epsilon}(s) \mu_{0}(\mathrm{~d} s)=\lim _{n \rightarrow \infty} \int_{(0,1]} f_{\epsilon}(s) \mu_{t_{n}}(\mathrm{~d} s) \geq \liminf _{n \rightarrow \infty} \int_{(\epsilon, 1]} s \mu_{t_{n}}(\mathrm{~d} s) .
$$

Let us estimate the last integral. We write

$$
\begin{aligned}
\int_{(\epsilon, 1]} s \mu_{t}(\mathrm{ds}) & =t \lambda^{-1} \int_{(\lambda \epsilon, \lambda]} s \nu_{1}(\mathrm{~d} s) \\
& =\frac{1}{2} t \lambda^{-1} \int_{(\lambda \epsilon, \lambda]} s \nu(\mathrm{d} s) .
\end{aligned}
$$

By the Fubini-Tonelli theorem, we get

$$
\int_{(\lambda \epsilon, \lambda]} s \nu(\mathrm{d} s)=\int_{\lambda \epsilon}^{\lambda} u^{-2} \int_{(0, u]} s^{2} \nu(\mathrm{d} s) \mathrm{d} u+\lambda K(\lambda)-\lambda \epsilon K(\lambda \epsilon) .
$$

Thus,

$$
2 \int_{(\epsilon, 1]} s \mu_{t}(\mathrm{~d} s)=t \lambda^{-1} \int_{\lambda \epsilon}^{\lambda} K(u) \mathrm{d} u+t K(\lambda)-t \epsilon K(\lambda \epsilon)
$$


Setting $z=1 / \lambda$, by (4.2) and (4.45), we obtain

$$
t K(\lambda) \approx t \varphi(z) \approx 1
$$

Moreover, since $\varphi$ is a 1-regularly varying function at infinity, we have

$$
t \epsilon K(\lambda \epsilon) \approx t \epsilon \varphi(z / \epsilon)=M \epsilon \frac{\varphi(z / \epsilon)}{\varphi(z)} \rightarrow M,
$$

as $z$ tends to infinity. Therefore, it remains to estimate the integral in (4.57). Using (4.2) we get

$$
\begin{aligned}
t \lambda^{-1} \int_{\lambda \epsilon}^{\lambda} K(u) \mathrm{d} u & \approx \frac{z}{\varphi(z)} \int_{\epsilon z^{-1}}^{z^{-1}} \varphi\left(u^{-1}\right) \mathrm{d} u \\
& =\frac{z}{\varphi(z)} \int_{z}^{\epsilon^{-1} z} u^{-2} \varphi(u) \mathrm{d} u \\
& =\frac{\phi^{\prime}(z)-\phi^{\prime}\left(\epsilon^{-1} z\right)}{z\left(-\phi^{\prime \prime}(z)\right)} .
\end{aligned}
$$

Since $-\phi^{\prime \prime}(s)=s^{-1} \ell(s)$ for a certain function $\ell$ slowly varying at infinity, by [3, Theorem 1.5.6],

$$
\frac{\phi^{\prime}(z)-\phi^{\prime}\left(\epsilon^{-1} z\right)}{z\left(-\phi^{\prime \prime}(z)\right)}=\int_{1}^{\epsilon^{-1}} \frac{\ell(z t)}{\ell(z)} \frac{\mathrm{d} t}{t} \rightarrow \log \epsilon^{-1},
$$

as $z$ tends to infinity. Hence,

$$
\liminf _{n \rightarrow \infty} \int_{(\epsilon, 1]} s \mu_{t_{n}}(\mathrm{~d} s) \gtrsim \log \epsilon^{-1},
$$

which by (4.56) implies (4.54).

Next, let us consider the case (i); that is, when $-\phi^{\prime \prime} \in \operatorname{WUSC}\left(\beta-2, C, x_{0}\right)$ with $C \geq 1$ and $\alpha \leq \beta<1$. We claim that for all $\epsilon \in(0,1)$,

$$
\int_{(0, \epsilon)} s^{2} \mu_{0}(\mathrm{~d} s)>0 .
$$

To see this, it is enough to show that there is $C>0$ such that for all $\epsilon \in(0,1]$ and $t \in\left(0,1 / \varphi\left(x_{0}\right)\right)$,

$$
\int_{(0, \epsilon)} s^{2} \mu_{t}(\mathrm{~d} s) \geq C \epsilon^{2-\alpha} .
$$

For the proof, we select a continuous function on $\mathbb{R}$ such that

$$
\mathbb{1}_{(-1,1)} \leq \eta \leq \mathbb{1}_{(-2,2)},
$$

and for each $\tau>0$ set

$$
\eta_{\tau}(x)=\eta\left(\tau^{-1} x\right)
$$


Since for $0<2 \tau<\epsilon$,

$$
\int_{(0, \infty)} s^{2}\left(\eta_{\epsilon}(s)-\eta_{\tau}(s)\right) \mu_{t}(\mathrm{~d} s)+\int_{(0,2 \tau)} s^{2} \eta_{\tau}(s) \mu_{t}(\mathrm{~d} s) \geq \int_{(0, \epsilon)} s^{2} \mu_{t}(\mathrm{~d} s)
$$

by (4.59) and (4.52),

$$
\int_{(0, \infty)} s^{2}\left(\eta_{\epsilon}(s)-\eta_{\tau}(s)\right) \mu_{0}(\mathrm{~d} s)+\limsup _{n \rightarrow \infty} \int_{(0, \infty)} s^{2} \eta_{\tau}(s) \mu_{t_{n}}(\mathrm{~d} s) \geq C \epsilon^{2-\alpha} .
$$

Since $Y_{t_{n}}$ and $Y_{0}$ are purely non-Gaussian, by [47, Theorem 8.7(2)],

$$
\lim _{\tau \rightarrow 0^{+}} \limsup _{n \rightarrow \infty} \int_{(-\tau, \tau)} s^{2} \mu_{t_{n}}(\mathrm{~d} s)=0
$$

thus,

$$
\int_{(0, \epsilon)} s^{2} \mu_{0}(\mathrm{~d} s) \geq C \epsilon^{2-\alpha}
$$

which entails (4.58).

We now turn to showing (4.59). We have

$$
\begin{aligned}
\int_{(0, \epsilon)} s^{2} \mu_{t}(\mathrm{~d} s) & =t \lambda^{-2} \int_{(0, \lambda \epsilon)} s^{2} \nu_{1}(\mathrm{~d} s) \\
& =\frac{1}{2} t \lambda^{-2} \int_{(0, \lambda \epsilon)} s^{2} \nu(\mathrm{d} s) \\
& =\frac{1}{2} t \epsilon^{2} K(\lambda \epsilon)
\end{aligned}
$$

thus, by (4.2) and the weak lower scaling property of $\varphi$,

$$
\begin{aligned}
\int_{(0, \epsilon)} s^{2} \mu_{t}(\mathrm{~d} s) & \gtrsim t \epsilon^{2} \varphi\left(\epsilon^{-1} \lambda^{-1}\right) \\
& \gtrsim t \epsilon^{2-\alpha} \varphi(1 / \lambda)
\end{aligned}
$$

which, together with the definition of $\lambda$, implies (4.59).

Since the support of the probability distribution of $Y_{0}$ is not the whole real line, by [42, Lemma 2.5], the inequality (4.58) implies that

$$
\int_{(0, \infty)} \min \{1, s\} \mu_{0}(\mathrm{~d} s)<\infty
$$

and the support of $Y_{0}$ equals $[\chi, \infty)$ where

$$
\chi=\gamma_{0}-\int_{(0, \infty)} s w(s) \mu_{0}(\mathrm{~d} s)=-\int_{(0,1]} s \mu_{0}(\mathrm{~d} s) .
$$


To conclude (4.49), it is enough to show that $\chi \leq-\tilde{\rho}$. Since $\rho\left(t_{n}\right) \leq \frac{1}{2} t_{n} \lambda_{n}^{-1} b_{\lambda_{n}}$, the latter can be deduced from

$$
\begin{aligned}
\chi & =-\lim _{n \rightarrow \infty} \frac{1}{2} t_{n} \lambda_{n}^{-1} b_{\lambda_{n}} \\
& =-\lim _{n \rightarrow \infty} \int_{(0,1]} s \mu_{t_{n}}(\mathrm{~d} s),
\end{aligned}
$$

where the last equality is a consequence of (4.48) since

$$
\begin{aligned}
\int_{(0,1]} s \mu_{t}(\mathrm{ds}) & =t \lambda^{-1} \int_{(0, \lambda]} s \nu_{1}(\mathrm{~d} s) \\
& =\frac{1}{2} t \lambda^{-1} \int_{(0, \lambda]} s \nu(\mathrm{d} s) .
\end{aligned}
$$

Therefore, the problem is reduced to showing (4.62). By the monotone convergence theorem and (4.52), we have

$$
\begin{aligned}
\chi & =-\lim _{\epsilon \rightarrow 0^{+}} \int_{(0,1]} f_{\epsilon}(s) \mu_{0}(\mathrm{~d} s) \\
& =-\lim _{\epsilon \rightarrow 0^{+}} \lim _{n \rightarrow \infty} \int_{(0,1]} f_{\epsilon}(s) \mu_{t_{n}}(\mathrm{~d} s)
\end{aligned}
$$

and

$$
\lim _{\epsilon \rightarrow 0^{+}} \int_{(0,1]} f_{\epsilon}(s) \mu_{t_{n}}(\mathrm{~d} s)=\int_{(0,1]} s \mu_{t_{n}}(\mathrm{~d} s),
$$

where $f_{\epsilon}$ is as in (4.55). Hence, we just need to justify the change in the order of limits. In view of the Moore-Osgood theorem [19, Chapter VII], it is enough to show that the limit in (4.65) is uniform with respect to $n \in \mathbb{N}$.

We write

$$
\begin{aligned}
\left|\int_{(0,1]} s \mu_{t}(\mathrm{~d} s)-\int_{(0,1]} f_{\epsilon}(s) \mu_{t}(\mathrm{~d} s)\right| & \leq \int_{(0, \epsilon / 2]} s \mu_{t}(\mathrm{~d} s)+\int_{(\epsilon / 2, \epsilon]}(\epsilon-s) \mu_{t}(\mathrm{~d} s) \\
& \leq \int_{(0, \epsilon]} s \mu_{t}(\mathrm{~d} s)
\end{aligned}
$$

By (4.63) and the Fubini-Tonelli theorem, we have

$$
\begin{aligned}
2 t^{-1} \lambda \int_{(0, \epsilon]} s \mu_{t}(\mathrm{~d} s)=\int_{(0, \lambda \epsilon]} s \nu(\mathrm{d} s) & =\int_{0}^{\lambda \epsilon} u^{-2} \int_{(0, u]} s^{2} \nu(\mathrm{d} s) \mathrm{d} u+\lambda \epsilon K(\lambda \epsilon) \\
& \approx \int_{0}^{\lambda \epsilon} \varphi\left(u^{-1}\right) \mathrm{d} u+\lambda \epsilon \varphi\left(\lambda^{-1} \epsilon^{-1}\right) .
\end{aligned}
$$

By almost monotonicity of $\varphi$,

$$
\int_{(0, \epsilon]} s \mu_{t}(\mathrm{~d} s) \approx t \lambda^{-1} \int_{0}^{\lambda \epsilon} \varphi\left(u^{-1}\right) \mathrm{d} u+t \epsilon \varphi\left(\lambda^{-1} \epsilon^{-1}\right)
$$




$$
\approx t \lambda^{-1} \int_{0}^{\lambda \epsilon} \varphi\left(u^{-1}\right) \mathrm{d} u .
$$

Now, setting $z=\varphi^{-1}(M / t)$, by (4.45), we get

$$
\begin{aligned}
t \lambda^{-1} \int_{0}^{\lambda \epsilon} \varphi\left(u^{-1}\right) \mathrm{d} u & =t \varphi^{-1}(M / t) \int_{0}^{\epsilon / \varphi^{-1}(M / t)} \varphi\left(u^{-1}\right) \mathrm{d} u \\
& \approx \frac{z}{\varphi(z)} \int_{0}^{\epsilon z^{-1}} \varphi\left(u^{-1}\right) \mathrm{d} u \\
& =\frac{z}{\varphi(z)} \int_{\epsilon^{-1} z}^{\infty} u^{-2} \varphi(u) \mathrm{d} u \\
& =\frac{\phi^{\prime}\left(\epsilon^{-1} z\right)}{z\left(-\phi^{\prime \prime}(z)\right)}
\end{aligned}
$$

In view of Proposition 2.4, by the upper scaling of $-\phi^{\prime \prime}$, there is $c>0$ such that for all $z>x_{0}$,

$$
\frac{\phi^{\prime}\left(\epsilon^{-1} z\right)}{z\left(-\phi^{\prime \prime}(z)\right)} \leq c \epsilon^{1-\beta} .
$$

Hence, the limit in (4.65) is uniform with respect to $n \in \mathbb{N}$, which justifies (4.62). This completes the proof of (4.49) and the theorem follows.

Theorem 4.13. Let $\mathbf{T}$ be a subordinator with the Laplace exponent $\phi$. Suppose that $\phi \in \operatorname{WLSC}\left(\alpha, c, x_{0}\right) \cap \operatorname{WUSC}\left(\beta, C, x_{0}\right)$ for some $c \in(0,1], C \geq 1, x_{0} \geq 0$ and $0<\alpha \leq \beta<1$. $W e$ also assume that $b=0$. Then for all $0<\chi_{1}<\chi_{2}$ there is $C^{\prime} \geq 1$ such that for all $t \in\left(0,1 / \varphi\left(x_{0}\right)\right)$ and $x>0$ satisfying

$$
\chi_{1} \leq x \phi^{-1}(1 / t) \leq \chi_{2},
$$

we have

$$
C^{\prime-1} \phi^{-1}(1 / t) \leq p(t, x) \leq C^{\prime} \phi^{-1}(1 / t) .
$$

Proof. First let us notice that Corollary 2.7 implies that $-\phi^{\prime \prime} \in \operatorname{WLSC}\left(\alpha-2, c, x_{0}\right) \cap$ $\operatorname{WUSC}\left(\beta-2, C, x_{0}\right)$. Therefore, the hypothesis of Theorem 4.11 is satisfied.

It is enough to show the first inequality in (4.68) since the latter is an easy consequence of (4.28) and Proposition 4.6. For $t \in\left(0,1 / \varphi\left(x_{0}\right)\right)$ and $M \geq 1$, we set

$$
x_{t}=t \phi^{\prime}\left(\varphi^{-1}(M / t)\right) \text {. }
$$

By Proposition 4.6, the function $\varphi^{-1}$ possesses the weak lower scaling property. Moreover, there is $C_{1} \geq 1$ such that for all $r>\varphi\left(x_{0}\right)$,

$$
C_{1}^{-1} \varphi^{-1}(r) \leq \phi^{-1}(r) \leq C_{1} \varphi^{-1}(r) .
$$

Hence, by Proposition 2.4, there is $C_{2} \geq 1$, such that

$$
x_{t} \leq C_{2} M^{1-1 / \beta} \frac{1}{\varphi^{-1}(1 / t)} \text {. }
$$


We select $M \geq 1$ satisfying

$$
C_{1} C_{2} M^{1-1 / \beta}<\chi_{1}
$$

Let $\rho_{1}=\rho_{0} / 2$ where $\rho_{0}$ is determined in Theorem 4.11. Then, by (4.69) and (4.70), we have

$$
\begin{aligned}
x_{t}-\frac{\rho_{1}}{\varphi^{-1}(1 / t)} & \leq C_{1} C_{2} M^{1-1 / \beta} \frac{1}{\phi^{-1}(1 / t)} \\
& <\frac{\chi_{1}}{\phi^{-1}(1 / t)} .
\end{aligned}
$$

Now set $\rho_{2}=C_{1} \chi_{2}$. Then, by (4.69), we have

$$
x_{t}+\frac{\rho_{2}}{\varphi^{-1}(1 / t)}>\frac{\rho_{2}}{C_{1} \phi^{-1}(1 / t)}=\frac{\chi_{2}}{\phi^{-1}(1 / t)} .
$$

Putting (4.72) and (4.71) together, we conclude that

$$
\left[\frac{\chi_{1}}{\phi^{-1}(1 / t)}, \frac{\chi_{2}}{\phi^{-1}(1 / t)}\right] \subseteq\left(x_{t}-\frac{\rho_{1}}{\varphi^{-1}(1 / t)}, x_{t}+\frac{\rho_{2}}{\varphi^{-1}(1 / t)}\right) .
$$

Therefore, by Theorem 4.11, for all $t \in\left(0,1 / \varphi\left(x_{0}\right)\right)$ and $x>0$ satisfying

$$
\chi_{1} \leq x \phi^{-1}(1 / t) \leq \chi_{2}
$$

we have

$$
p(t, x) \gtrsim \varphi^{-1}(1 / t) .
$$

In view of (4.69), this completes the proof of the theorem.

Proposition 4.14. Let $\mathbf{T}$ be a subordinator with the Laplace exponent $\phi$. Suppose that $-\phi^{\prime \prime} \in \mathrm{WLSC}\left(\alpha-2, c, x_{0}\right)$ for some $c \in(0,1], x_{0} \geq 0$ and $\alpha>0$, and assume that one of the following conditions holds true:

(i) $-\phi^{\prime \prime} \in \operatorname{WUSC}\left(\beta-2, C, x_{0}\right)$ for some $C \geq 1$ and $\alpha \leq \beta<1$, or

(ii) $-\phi^{\prime \prime}$ is a function regularly varying at infinity with index -1 . If $x_{0}=0$, we also assume that $-\phi^{\prime \prime}$ is regularly varying at zero with index -1 .

We also assume that the Lévy measure $\nu(\mathrm{d} x)$ has an almost monotone density $\nu(x)$. Then the probability distribution of $T_{t}$ has a density $p(t, \cdot)$. Moreover, there are $M_{0}>1, \rho_{0}>0$ and $C>0$ such that for all $t \in\left(0,1 / \varphi\left(x_{0}\right)\right)$ and

$$
x \geq 2 t \phi^{\prime}\left(\varphi^{-1}\left(M_{0} / t\right)\right)+\frac{2 \rho_{0}}{\varphi^{-1}(1 / t)},
$$

we have

$$
p(t, x) \geq C t \nu(x)
$$

Proof. Let $\lambda>0$. We begin by decomposing the Lévy measure $\nu(\mathrm{d} x)$. Let $\nu_{1}(\mathrm{~d} x)=$ $\nu_{1}(x) \mathrm{d} x$ and $\nu_{2}(\mathrm{~d} x)=\nu_{2}(x) \mathrm{d} x$ where

$$
\nu_{1}(x)=\nu(x)-\nu_{2}(x) \quad \text { and } \quad \nu_{2}(x)=\frac{1}{2} \nu(x) \mathbb{1}_{[\lambda, \infty)}(x) .
$$


For $u>0$, we set

$$
\phi_{1}(u)=b u+\int_{(0, \infty)}\left(1-e^{-u s}\right) \nu_{1}(\mathrm{~d} s) \quad \text { and } \quad \phi_{2}(u)=\int_{(0, \infty)}\left(1-e^{-u s}\right) \nu_{2}(\mathrm{~d} s) .
$$

Let $\mathbf{T}^{(j)}$ be the Lévy process having the Laplace exponent $\phi_{j}$, for $j \in\{1,2\}$. Since $\frac{1}{2} \nu \leq$ $\nu_{1} \leq \nu$, we have

$$
\frac{1}{2} \phi \leq \phi_{1} \leq \phi
$$

and for all $n \in \mathbb{N}$,

$$
\frac{1}{2}(-1)^{n+1} \phi^{(n)} \leq(-1)^{n+1} \phi_{1}^{(n)} \leq(-1)^{n+1} \phi^{(n)}
$$

Thus,

$$
\frac{1}{2} \varphi \leq \varphi_{1} \leq \varphi
$$

and so for all $u>0$,

$$
\varphi_{1}^{-1}(u / 2) \leq \varphi^{-1}(u) \leq \varphi_{1}^{-1}(u)
$$

In particular, $-\phi_{1}^{\prime \prime}$ has the weak lower scaling property. Therefore, by Theorem $3.3, T_{t}^{(1)}$ and $T_{t}$ are absolutely continuous. Let us denote by $p(t, \cdot)$ and $p^{(1)}(t, \cdot)$ the densities of $T_{t}$ and $T_{t}^{(1)}$, respectively. Observe that $\mathbf{T}^{(2)}$ is a compound Poisson process with the probability distribution denoted by $P_{t}(\mathrm{~d} x)$. By [47, Remark 27.3],

$$
P_{t}(\mathrm{~d} x) \geq t e^{-t \nu_{2}(\mathbb{R})} \nu_{2}(x) \mathrm{d} x \text {. }
$$

We apply Theorem 4.11 to the process $\mathbf{T}^{(1)}$. For $t>0$, we set

$$
x_{t}=t \phi_{1}^{\prime}\left(\varphi_{1}^{-1}\left(M_{0} / t\right)\right) \text {. }
$$

Then there are $C>0$ and $\rho_{0}>0$, such that for all $t \in\left(0,1 / \varphi\left(x_{0}\right)\right)$ and $x \geq 0$ satisfying

$$
x_{t}-\frac{\rho_{0}}{\varphi_{1}^{-1}(1 / t)} \leq x \leq x_{t}+\frac{\rho_{0}}{\varphi_{1}^{-1}(1 / t)},
$$

we have

$$
p^{(1)}(t, x) \geq C \varphi_{1}^{-1}(1 / t)
$$

Therefore, if

$$
\lambda=x_{t}+\frac{\rho_{0}}{\varphi_{1}^{-1}(1 / t)}
$$

then

$$
\int_{0}^{\lambda} p^{(1)}(t, x) \mathrm{d} x \gtrsim 1
$$

Next, if $\lambda \geq \rho_{0} / \varphi^{-1}(1 / t)$, then, by (4.2),

$$
t \nu_{2}(\mathbb{R})=\frac{1}{2} t \int_{\lambda}^{\infty} \nu(x) \mathrm{d} x
$$




$$
\begin{aligned}
& \leq \frac{1}{2} \operatorname{th}\left(\rho_{0} / \varphi^{-1}(1 / t)\right) \\
& \lesssim t h\left(1 / \varphi^{-1}(1 / t)\right) \\
& \lesssim 1,
\end{aligned}
$$

where the penultimate inequality follows either by monotonicity of $h$ or by [23, Lemma 2.1 (4)]. Finally, by (4.75) and (4.77), for $x \geq 2 \lambda$ we can compute

$$
\begin{aligned}
p(t, x) & =\int_{\mathbb{R}} p^{(1)}(t, x-y) P_{t}(\mathrm{~d} y) \\
& \gtrsim t \int_{\mathbb{R}} p^{(1)}(t, x-y) \nu_{2}(y) \mathrm{d} y \\
& =\frac{1}{2} t \int_{\lambda}^{x} p^{(1)}(t, x-y) \nu(y) \mathrm{d} y .
\end{aligned}
$$

Hence, by the monotonicity of $\nu$, we get

$$
\begin{aligned}
p(t, x) & \gtrsim t \nu(x) \int_{0}^{x-\lambda} p^{(1)}(t, y) \mathrm{d} y \\
& \geq t \nu(x) \int_{0}^{\lambda} p^{(1)}(t, y) \mathrm{d} y \\
& \gtrsim t \nu(x),
\end{aligned}
$$

where in the last estimate we have used (4.76). Using (4.73) and (4.74), we can easily show that

$$
\lambda=x_{t}+\frac{\rho_{0}}{\varphi_{1}^{-1}(1 / t)} \leq t \phi^{\prime}\left(\varphi^{-1}\left(M_{0} / t\right)\right)+\frac{\rho_{0}}{\varphi^{-1}(1 / t)}
$$

and the proposition follows.

\subsection{Sharp two-sided estimates}

In this section we present sharp two-sided estimates on the density $p(t, \cdot)$ assuming both the weak lower and upper scaling properties on $-\phi^{\prime \prime}$. First, following [6, Lemma 13], we prove an auxiliary result.

Proposition 4.15. Assume that the Lévy measure $\nu(\mathrm{d} x)$ has an almost monotone density $\nu(x)$. Suppose that $-\phi^{\prime \prime} \in \operatorname{WUSC}\left(\gamma, C, x_{0}\right)$ for some $C \geq 1, x_{0} \geq 0$ and $\gamma<0$. Then there are $a \in(0,1]$ and $c \in(0,1]$ such that for all $0<x<a / x_{0}$,

$$
\nu(x) \geq c x^{-3}\left(-\phi^{\prime \prime}(1 / x)\right) .
$$

Proof. Let $a \in(0,1]$. Recall that by (4.30) we have $\nu(s) \leq C_{1} s^{-3}\left(-\phi^{\prime \prime}(1 / s)\right)$ for any $s>0$. Hence, for any $u>0$,

$$
\begin{aligned}
-\phi^{\prime \prime}(u) & =\int_{0}^{a u^{-1}} s^{2} e^{-u s} \nu(s) \mathrm{d} s+\int_{a u^{-1}}^{\infty} s^{2} e^{-u s} \nu(s) \mathrm{d} s \\
& \leq C_{1} \int_{0}^{a u^{-1}} s^{-1} e^{-u s}\left(-\phi^{\prime \prime}(1 / s)\right) \mathrm{d} s+C_{2} \nu\left(a u^{-1}\right) \int_{a u^{-1}}^{\infty} s^{2} e^{-u s} \mathrm{~d} s
\end{aligned}
$$


where $C_{2}$ is a constant from the almost monotonicity of $\nu$. If $u>x_{0}$, then by the scaling property of $-\phi^{\prime \prime}$ we obtain

$$
\begin{aligned}
C_{1} \int_{0}^{a u^{-1}} s^{-1} e^{-u s}\left(-\phi^{\prime \prime}(1 / s)\right) \mathrm{d} s & \leq C \int_{0}^{a u^{-1}} s^{-1} e^{-u s}(s u)^{-\gamma}\left(-\phi^{\prime \prime}(u)\right) \mathrm{d} s \\
& \leq C\left(-\phi^{\prime \prime}(u)\right) \int_{0}^{a} s^{-1-\gamma} e^{-s} \mathrm{~d} s
\end{aligned}
$$

By selecting $a \in(0,1]$ such that

$$
2 C \int_{0}^{a} s^{-1-\gamma} e^{-s} \mathrm{~d} s \leq 1
$$

we get

$$
\int_{0}^{a u^{-1}} s^{-1} e^{-u s}\left(-\phi^{\prime \prime}(1 / s)\right) \mathrm{d} s \leq \frac{1}{2}\left(-\phi^{\prime \prime}(u)\right)
$$

Since

$$
\int_{a u^{-1}}^{\infty} s^{2} e^{-u s} \mathrm{~d} s=u^{-3} e^{-a}\left(a^{2}+2 a+2\right)
$$

by (4.78) we obtain

$$
\nu\left(a u^{-1}\right) \geq \frac{e^{a}}{2\left(a^{2}+2 a+2\right)} u^{3}\left(-\phi^{\prime \prime}(u)\right),
$$

provided that $u>x_{0}$. Now, by the monotonicity of $-\phi^{\prime \prime}$ we conclude the proof.

In view of Propositions 2.3 and 2.4, we immediately obtain the following corollary.

Corollary 4.16. Assume that the Lévy measure $\nu(\mathrm{d} x)$ has an almost monotone density $\nu(x)$. Suppose that $b=0$ and $\phi \in \operatorname{WLSC}\left(\alpha, c, x_{0}\right) \cap \operatorname{WUSC}\left(\beta, C, x_{0}\right)$ for some $c \in(0,1]$, $C \geq 1, x_{0} \geq 0$ and $0<\alpha \leq \beta<1$. Then there are $a \in(0,1]$ and $c^{\prime} \in(0,1]$ such that for all $0<x<a / x_{0}$,

$$
\nu(x) \geq c^{\prime} x^{-1} \phi(1 / x)
$$

We are now ready to prove our main result in this section.

Theorem 4.17. Let $\mathbf{T}$ be a subordinator with the Laplace exponent $\phi$. Suppose that $\phi \in \operatorname{WLSC}\left(\alpha, c, x_{0}\right) \cap \operatorname{WUSC}\left(\beta, C, x_{0}\right)$ for some $c \in(0,1], C \geq 1, x_{0} \geq 0$ and $0<\alpha \leq \beta<1$. We also assume that $b=0$ and that the Lévy measure $\nu(\mathrm{d} x)$ has an almost monotone density $\nu(x)$. Then there is $x_{1} \in(0, \infty]$ such that for all $t \in\left(0,1 / \varphi\left(x_{0}\right)\right)$ and $x \in\left(0, x_{1}\right)$,

$$
p(t, x) \approx \begin{cases}\left(t\left(-\phi^{\prime \prime}(w)\right)\right)^{-\frac{1}{2}} \exp \left\{-t\left(\phi(w)-w \phi^{\prime}(w)\right)\right\} & \text { if } 0<x \phi^{-1}(1 / t) \leq 1 \\ t x^{-1} \phi(1 / x) & \text { if } 1<x \phi^{-1}(1 / t),\end{cases}
$$

where $w=\left(\phi^{\prime}\right)^{-1}(x / t)$. If $x_{0}=0$ then $x_{1}=\infty$. 
Proof. First let us note that by Corollary 2.7, $-\phi^{\prime \prime} \in \operatorname{WLSC}\left(\alpha-2, c, x_{0}\right) \cap \operatorname{WUSC}(\beta-$ $\left.2, C, x_{0}\right)$. Therefore, we are in position to apply Proposition 4.14. By Corollary 3.7, for $\chi_{1}=\min \{1, \delta\}$, we have

$$
p(t, x) \approx\left(t\left(-\phi^{\prime \prime}(w)\right)\right)^{-\frac{1}{2}} \exp \left\{-t\left(\phi(w)-w \phi^{\prime}(w)\right)\right\},
$$

whenever $0<x \phi^{-1}(1 / t) \leq \chi_{1}$. Next, let $M_{0}^{\prime}$ be $M_{0}$ determined by Proposition 4.14. By Proposition 2.4, (4.2) and monotonicity of $\varphi^{-1}$, for $t \in\left(0,1 / \varphi\left(x_{0}\right)\right)$, we get

$$
t \phi^{\prime}\left(\psi^{-1}(1 / t)\right) \lesssim \frac{1}{\psi^{-1}(1 / t)} \quad \text { and } \quad t \phi^{\prime}\left(\varphi^{-1}\left(M_{0}^{\prime} / t\right)\right) \lesssim \frac{1}{\varphi^{-1}(1 / t)}
$$

thus, by Propositions 4.3 and 4.6, there is $C_{1}>0$ such that

$$
2 e t \phi^{\prime}\left(\psi^{-1}(1 / t)\right) \leq C_{1} \frac{1}{\phi^{-1}(1 / t)}
$$

and

$$
2 t \phi^{\prime}\left(\varphi^{-1}\left(M_{0}^{\prime} / t\right)\right)+\frac{2 \rho_{0}^{\prime}}{\varphi^{-1}(1 / t)} \leq C_{1} \frac{1}{\phi^{-1}(1 / t)}
$$

where $\rho_{0}^{\prime}$ is the value of $\rho_{0}$ determined in Proposition 4.14. Let $\chi_{2}=\max \left\{1, C_{1}, \chi_{1}\right\}$. By Proposition 4.14 and Corollary 4.16, there is $a \in(0,1]$ such that if $x \phi^{-1}(1 / t)>\chi_{2}$ and $0<x<a / x_{0}$, then

$$
\begin{aligned}
p(t, x) & \gtrsim t \nu(x) \\
& \gtrsim t x^{-1} \phi(1 / x) .
\end{aligned}
$$

Furthermore, by (4.29), if $x \phi^{-1}(1 / t)>\chi_{2}$, then

$$
\begin{aligned}
p(t, x) & \lesssim t \eta(x) \\
& \lesssim t x^{-1} \phi(1 / x),
\end{aligned}
$$

where in the last step we have also used (4.13). Lastly, by Theorem 4.13 there is $C_{2} \geq 1$ such that for all $t \in\left(0,1 / \varphi\left(x_{0}\right)\right)$ and $x>0$ satisfying

$$
\chi_{1} \leq x \phi^{-1}(1 / t) \leq \chi_{2}
$$

we have

$$
C_{2}^{-1} \phi^{-1}(1 / t) \leq p(t, x) \leq C_{2} \phi^{-1}(1 / t) .
$$

We next claim that the following holds true.

Claim 4.18. There exist $0<c_{1} \leq 1 \leq c_{2}$ such that for all $t \in\left(0, c_{1} / \varphi\left(x_{0}\right)\right)$ and $x>0$ satisfying

$$
\chi_{1} \leq x \phi^{-1}(1 / t) \leq \chi_{2}
$$

we have

$$
t \phi^{\prime}\left(\phi^{-1}\left(c_{2} / t\right)\right) \leq x \leq t \phi^{\prime}\left(\phi^{-1}\left(c_{1} / t\right)\right)
$$


By Proposition 4.6, there is $C_{3} \geq 1$ such that for $r>\varphi\left(x_{0}\right)$,

$$
C_{3}^{-1} \varphi^{-1}(r) \leq \phi^{-1}(r) \leq C_{3} \varphi^{-1}(r) .
$$

Let $c_{2}=\left(\chi_{1} c^{\prime} C_{3}^{-2}\right)^{-\beta /(1-\beta)} \in[1, \infty)$, where $c^{\prime}$ is taken from (4.15). Then

$$
c_{2}^{-1} \phi^{-1}\left(c_{2} / t\right) \geq C_{3}^{-2} c^{\prime} c_{2}^{-1+1 / \beta} \phi^{-1}(1 / t)=\chi_{1}^{-1} \phi^{-1}(1 / t) .
$$

Consequently, by Proposition 2.3,

$$
\begin{aligned}
x \geq \frac{\chi_{1}}{\phi^{-1}(1 / t)} & \geq t \frac{\phi\left(\phi^{-1}\left(c_{2} / t\right)\right)}{\phi^{-1}\left(c_{2} / t\right)} \\
& \geq t \phi^{\prime}\left(\phi^{-1}\left(c_{2} / t\right)\right) .
\end{aligned}
$$

Moreover, there is $C_{4} \geq 1$ such that $C_{4} x \phi^{\prime}(x) \geq \phi(x)$ provided that $x>x_{0}$. Therefore, if $\chi_{2} \leq C_{4}^{-1}$, then

$$
\begin{aligned}
\frac{\chi_{2}}{\phi^{-1}(1 / t)} & =\chi_{2} t \frac{\phi\left(\phi^{-1}(1 / t)\right)}{\phi^{-1}(1 / t)} \\
& \leq t \phi^{\prime}\left(\phi^{-1}(1 / t)\right),
\end{aligned}
$$

which yields (4.80) with $c_{1}=1$. Otherwise, if $\chi_{2}>C_{4}^{-1}$, then we set $c_{1}=$ $\left(C_{4} \chi_{2} C_{3}^{2}\left(c^{\prime}\right)^{-1}\right)^{-\beta /(1-\beta)} \in(0,1]$. Hence, by Proposition 4.6, for all $t \in\left(0, c_{1} / \varphi\left(x_{0}\right)\right)$,

$$
\frac{C_{4} \chi_{2}}{c_{1}} \phi^{-1}\left(c_{1} / t\right) \leq C_{4} \chi_{2} C_{3}^{2}\left(c^{\prime}\right)^{-1} c_{1}^{-1+1 / \beta} \phi^{-1}(1 / t)=\phi^{-1}(1 / t) .
$$

Therefore,

$$
\begin{aligned}
x & \leq \frac{\chi_{2}}{\phi^{-1}(1 / t)} \\
& \leq t \frac{\chi_{2}}{c_{1}} \cdot \frac{\phi^{-1}\left(c_{1} / t\right)}{\phi^{-1}(1 / t)} \cdot \frac{\phi\left(\phi^{-1}\left(c_{1} / t\right)\right)}{\phi^{-1}\left(c_{1} / t\right)} \\
& \leq t \phi^{\prime}\left(\phi^{-1}\left(c_{1} / t\right)\right),
\end{aligned}
$$

which, combined with (4.81) and (4.82), implies (4.80).

Now, using Claim 4.18 and Propositions 4.3 and 4.6, we deduce that for $t \in\left(0, c_{1} / \varphi\left(x_{0}\right)\right)$ and $\chi_{1} \leq x \phi^{-1}(1 / t) \leq \chi_{2}$,

$$
w \leq \phi^{-1}\left(c_{2} / t\right) \lesssim \phi^{-1}(1 / t)
$$

and

$$
w \geq \phi^{-1}\left(c_{1} / t\right) \gtrsim \phi^{-1}(1 / t)
$$

Hence, $t w \phi^{\prime}(w) \approx 1$ and

$$
\exp \left\{-t\left(\phi(w)-w \phi^{\prime}(w)\right)\right\} \approx 1 .
$$

Next, by Propositions 2.4 and 2.1,

$$
w^{2}\left(-\phi^{\prime \prime}(w)\right) \approx w \phi^{\prime}(w)
$$


thus, by (4.83) and (4.84), we obtain

$$
\frac{1}{\sqrt{t\left(-\phi^{\prime \prime}(w)\right)}} \approx \frac{w}{\sqrt{t w \phi^{\prime}(w)}} \approx \phi^{-1}(1 / t),
$$

which, together with (4.85), implies that

$$
\left(t\left(-\phi^{\prime \prime}(w)\right)\right)^{-\frac{1}{2}} \exp \left\{-t\left(\phi(w)-w \phi^{\prime}(w)\right)\right\} \approx \phi^{-1}(1 / t)
$$

for $t \in\left(0, c_{1} / \varphi\left(x_{0}\right)\right)$ and $\chi_{1} \leq x \phi^{-1}(1 / t) \leq \chi_{2}$. In view of (4.79), the theorem follows in the case $x_{0}=0$. Now, it remains to observe that in the case $x_{0}>0$ we may use positivity and continuity to conclude the claim for all $t \in\left(0,1 / \varphi\left(x_{0}\right)\right)$.

\section{Applications}

\subsection{Subordination}

Let $(\mathscr{X}, \tau)$ be a locally compact separable metric space with a Radon measure $\mu$ having full support on $\mathscr{X}$. Assume that $\left(X_{t}: t \geq 0\right)$ is a homogeneous in time Markov process on $\mathscr{X}$ with density $h(t, \cdot, \cdot)$; that is,

$$
\mathbb{P}\left(X_{t} \in B \mid X_{0}=x\right)=\int_{B} h(t, x, y) \mu(\mathrm{d} y)
$$

for any Borel set $B \subset \mathscr{X}, x \in \mathscr{X}$ and $t>0$. Assume that for all $t>0$ and $x, y \in \mathscr{X}$,

$$
t^{-\frac{n}{\gamma}} \Phi_{1}\left(\tau(x, y) t^{-\frac{1}{\gamma}}\right) \leq h(t, x, y) \leq t^{-\frac{n}{\gamma}} \Phi_{2}\left(\tau(x, y) t^{-\frac{1}{\gamma}}\right)
$$

where $n$ and $\gamma$ are some positive constants, $\Phi_{1}$ and $\Phi_{2}$ are nonnegative nonincreasing function on $[0, \infty)$ such that $\Phi_{1}(1)>0$ and

$$
\sup _{s \geq 0} \Phi_{2}(s)(1+s)^{n+\gamma}<\infty
$$

By $H(t, x, y)$ we denote the heat kernel for the subordinate process $\left(X_{T_{t}}: t \geq 0\right)$; that is,

$$
H(t, x, y)=\int_{0}^{\infty} h(s, x, y) G(t, \mathrm{~d} s),
$$

where

$$
G(t, s)=\mathbb{P}\left(T_{t} \geq s\right) .
$$

Suppose that $\phi \in \operatorname{WLSC}\left(\alpha, c, x_{0}\right) \cap \operatorname{WUSC}\left(\beta, C, x_{0}\right)$ for some $c \in(0,1], C \geq 1, x_{0}>0$ and $0<\alpha \leq \beta<1$. We also assume that

$$
\lim _{x \rightarrow \infty} \phi^{\prime}(x)=b=0
$$

and that the Lévy measure $\nu(\mathrm{d} x)$ has an almost monotone density $\nu(x)$. 
Claim 5.1. For all $x, y \in \mathscr{X}$ satisfying $\tau(x, y)^{-\gamma}>x_{0}$ and any $t \in\left(0,1 / \varphi\left(x_{0}\right)\right)$,

$$
H(t, x, y) \approx \begin{cases}t \phi\left(\tau(x, y)^{-\gamma}\right) \tau(x, y)^{-n} & \text { if } 0<t \phi\left(\tau(x, y)^{-\gamma}\right) \leq 1, \\ \left(\phi^{-1}(1 / t)\right)^{\frac{n}{\gamma}} & \text { if } 1 \leq t \phi\left(\tau(x, y)^{-\gamma}\right) .\end{cases}
$$

By Proposition 2.3, $\phi^{\prime} \in \mathrm{WLSC}\left(\alpha-1, c, x_{0}\right) \cap \operatorname{WUSC}\left(\beta-1, C, x_{0}\right)$. Let $0<r<\phi^{\prime}\left(x_{0}^{+}\right)$. If $0<\lambda \leq C$, then by setting

$$
D=C^{\frac{1}{1-\beta}} \lambda^{-\frac{1}{1-\beta}}
$$

the weak upper scaling property of $\phi^{\prime}$ implies that

$$
\lambda r=\lambda \phi^{\prime}\left(\left(\phi^{\prime}\right)^{-1}(r)\right) \geq \phi^{\prime}\left(D\left(\phi^{\prime}\right)^{-1}(r)\right) .
$$

Therefore,

$$
\left(\phi^{\prime}\right)^{-1}(\lambda r) \leq C^{\frac{1}{1-\beta}} \lambda^{-\frac{1}{1-\beta}}\left(\phi^{\prime}\right)^{-1}(r) .
$$

Analogously, we can prove the lower estimate: If $0<\lambda \leq c$, then by setting

$$
D=c^{\frac{1}{1-\alpha}} \lambda^{-\frac{1}{1-\alpha}}
$$

we obtain

$$
\lambda r=\lambda \phi^{\prime}\left(\left(\phi^{\prime}\right)^{-1}(r)\right) \leq \phi^{\prime}\left(D\left(\phi^{\prime}\right)^{-1}(r)\right)
$$

and, consequently,

$$
\left(\phi^{\prime}\right)^{-1}(\lambda r) \geq c^{\frac{1}{1-\alpha}} \lambda^{-\frac{1}{1-\alpha}}\left(\phi^{\prime}\right)^{-1}(r) .
$$

Since $\left(\phi^{\prime}\right)^{-1}$ is nonincreasing, the last inequality is valid for all $0<\lambda \leq 1$. Let

$$
\begin{aligned}
H(t, x, y) & =\left(\int_{0}^{\frac{1}{\phi^{-1}(1 / t)}}+\int_{\frac{1}{\phi^{-1(1 / t)}}}^{\infty}\right) h(s, x, y) G(t, \mathrm{~d} s) \\
& =I_{1}(t, x, y)+I_{2}(t, x, y) .
\end{aligned}
$$

By Theorem 4.17,

$$
I_{1} \approx \frac{1}{\phi^{-1}(1 / t)} \int_{0}^{1} h\left(\frac{u}{\phi^{-1}(1 / t)}, x, y\right) \frac{1}{\sqrt{t\left(-\phi^{\prime \prime}(w)\right)}} \exp \left(-t\left(\phi(w)-w \phi^{\prime}(w)\right)\right) \mathrm{d} u
$$

where

$$
w=\left(\phi^{\prime}\right)^{-1}\left(\frac{u}{t \phi^{-1}(1 / t)}\right)
$$

Recall that, by Proposition 2.3, for all $r>x_{0}$ we have

$$
r \phi^{\prime}(r) \leq \phi(r) \leq C_{1} r \phi^{\prime}(r) .
$$

We can assume that

$$
t \phi\left(2\left(C C_{1}\right)^{\frac{1}{1-\beta}} x_{0}\right)<1
$$


By (5.6) and the weak upper scaling of $\phi^{\prime}$, we get

$$
\begin{aligned}
\phi^{\prime}\left(\phi^{-1}(1 / t)\right) \leq \frac{1}{t \phi^{-1}(1 / t)} & \leq C_{1} \phi^{\prime}\left(\phi^{-1}(1 / t)\right) \\
& \leq \phi^{\prime}\left(\left(C C_{1}\right)^{-\frac{1}{1-\beta}} \phi^{-1}(1 / t)\right)
\end{aligned}
$$

thus,

$$
\left(\phi^{\prime}\right)^{-1}\left(\frac{1}{t \phi^{-1}(1 / t)}\right) \approx \phi^{-1}(1 / t) .
$$

Hence, by (5.3) and (5.4), we obtain

$$
u^{-\frac{1}{1-\alpha}} \phi^{-1}(1 / t) \lesssim w \lesssim u^{-\frac{1}{1-\beta}} \phi^{-1}(1 / t), \quad u \in(0,1] .
$$

Moreover, since $w>x_{0}$, by (5.6) and Proposition 4.6,

$$
\begin{aligned}
w \phi^{\prime}(w) \gtrsim \phi(w)-w \phi^{\prime}(w) & =\int_{0}^{w} \varphi(u) \frac{\mathrm{d} u}{u} \\
& \geq \int_{x_{0}}^{w} \varphi(u) \frac{\mathrm{d} u}{u} \gtrsim w \phi^{\prime}(w) .
\end{aligned}
$$

Thus, (5.7) entails that

$$
u^{-\frac{\alpha}{1-\alpha}} \lesssim t\left(\phi(w)-w \phi^{\prime}(w)\right) \lesssim u^{-\frac{\beta}{1-\beta}}, \quad u \in(0,1] .
$$

Next, by Proposition 4.6 and (5.6), we get

$$
\frac{1}{\sqrt{t\left(-\phi^{\prime \prime}(w)\right)}} \approx \frac{w}{\sqrt{t \phi(w)}} \approx \sqrt{u^{-1} \phi^{-1}(1 / t) w} .
$$

Therefore, by (5.7),

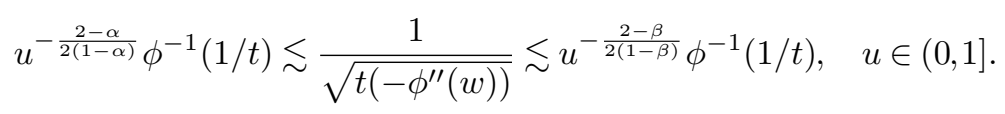

Now, by (5.5) and (5.1) together with (5.8) and (5.9), we can estimate

$$
I_{1} \lesssim\left(\phi^{-1}(1 / t)\right)^{\frac{n}{\gamma}} \int_{0}^{1} \Phi_{2}\left(u^{-\frac{1}{\gamma}} A^{\frac{1}{\gamma}}\right) u^{-\frac{n}{\gamma}-\frac{2-\beta}{2(1-\beta)}} \exp \left(-C^{\prime \prime} u^{-\frac{\alpha}{1-\alpha}}\right) \mathrm{d} u
$$

and

$$
I_{1} \gtrsim\left(\phi^{-1}(1 / t)\right)^{\frac{n}{\gamma}} \int_{0}^{1} \Phi_{1}\left(u^{-\frac{1}{\gamma}} A^{\frac{1}{\gamma}}\right) u^{-\frac{n}{\gamma}-\frac{2-\alpha}{2(1-\alpha)}} \exp \left(-C^{\prime} u^{-\frac{\beta}{1-\beta}}\right) \mathrm{d} u,
$$

where

$$
A=\tau(x, y)^{\gamma} \phi^{-1}(1 / t) .
$$

Suppose that $A \leq 1$. Since $\Phi_{1}$ and $\Phi_{2}$ are nonincreasing, by (5.10) and (5.11), we easily see that

$$
I_{1} \approx\left(\phi^{-1}(1 / t)\right)^{\frac{n}{\gamma}}
$$


We also have

$$
I_{2} \lesssim \int_{\frac{1}{\phi^{-1}(1 / t)}}^{\infty} s^{-\frac{n}{\gamma}} p(t, s) \mathrm{d} s \lesssim\left(\phi^{-1}(1 / t)\right)^{\frac{n}{\gamma}}
$$

Therefore,

$$
H(t, x, y) \approx\left(\phi^{-1}(1 / t)\right)^{\frac{n}{\gamma}}
$$

We now turn to the case $A>1$. By (5.2) and (5.10),

$$
\begin{aligned}
I_{1} & \lesssim\left(\phi^{-1}(1 / t)\right)^{\frac{n}{\gamma}} A^{-\frac{n}{\gamma}-1} \int_{0}^{1} u^{-\frac{\beta}{2(1-\beta)}} \exp \left(-C^{\prime \prime} u^{-\frac{\alpha}{1-\alpha}}\right) \mathrm{d} u \\
& \lesssim A^{-1} \tau(x, y)^{-n} .
\end{aligned}
$$

It remains to estimate $I_{2}$. Let us observe that for all $r>x_{0}$, if $u \geq 1$, then by the weak upper scaling of $\phi$, we have

$$
\phi(r) \leq \phi(r u) \leq C u^{\beta} \phi(r) .
$$

On the other hand, if $0<u \leq 1$, then by (2.10) and the monotonicity of $\phi$, we get

$$
u \phi(r) \leq \phi(r u) \leq \phi(r) .
$$

Therefore, for all $u>0$ and $r>x_{0}$,

$$
\min \{1, u\} \phi(r) \leq \phi(r u) \leq C \max \left\{1, u^{\beta}\right\} \phi(r) .
$$

Since $\tau(x, y)^{-\gamma}>x_{0}$, by Theorem 4.17, (5.1) and estimates (5.13), we get

$$
I_{2} \lesssim t \phi\left(\tau(x, y)^{-\gamma}\right) \tau(x, y)^{-n} \int_{1 / A}^{\infty} \Phi_{2}\left(u^{-\frac{1}{\gamma}}\right) u^{-\frac{n}{\gamma}-1} \max \left\{1, u^{-\beta}\right\} \mathrm{d} u
$$

and

$$
I_{2} \gtrsim t \phi\left(\tau(x, y)^{-\gamma}\right) \tau(x, y)^{-n} \int_{1 / A}^{\infty} \Phi_{1}\left(u^{-\frac{1}{\gamma}}\right) u^{-\frac{n}{\gamma}-1} \min \{1, u\} \mathrm{d} u .
$$

By (5.2), we have

$$
\int_{0}^{1} \Phi_{2}\left(u^{-\frac{1}{\gamma}}\right) u^{-\frac{n}{\gamma}-\beta-1} \mathrm{~d} u \lesssim \int_{0}^{1} u^{-\beta} \mathrm{d} u<\infty
$$

thus,

$$
I_{2} \approx t \phi\left(\tau(x, y)^{-\gamma}\right) \tau(x, y)^{-n} .
$$

Finally, since $A>1$, by (2.10), we have

$$
t \phi\left(\tau(x, y)^{-\gamma}\right)=t \phi\left(A^{-1} \phi^{-1}(1 / t)\right) \geq A^{-1} ;
$$

hence, by (5.12),

$$
I_{1} \lesssim t \phi\left(\tau(x, y)^{-\gamma}\right) \tau(x, y)^{-n}
$$

proving the claim. 
Example 5.2. Let $(\mathscr{X}, \tau)$ be a nested fractal with the geodesic metric on $\mathscr{X}$. Let $d_{w}$ and $d_{f}$ be the walk dimension and the Hausdorff dimension of $\mathscr{X}$, respectively. Let $\left(X_{t}: t \geq 0\right)$ be the diffusion on $\mathscr{X}$ constructed in [2, Section 7]. By [2, Theorem 8.18], the corresponding heat kernel satisfies (5.1) with $n=d_{f}, \gamma=d_{w}$, and

$$
\Phi_{1}(s)=\Phi_{2}(s)=\exp \left(-s^{\frac{\gamma}{\gamma-1}}\right) .
$$

Let $\mathbf{T}$ be a subordinator with the Laplace exponent

$$
\phi(s)=s^{\alpha} \log ^{\sigma}(2+s),
$$

where $\alpha \in(0,1)$ and $\sigma \in \mathbb{R}$. Then, by Claim 5.1 , the process $\left(X_{T_{t}}: t \geq 0\right)$ has density $H(t, x, y)$ such that for all $x, y \in \mathscr{X}$ and $t>0$,

•. if $t>\tau(x, y)^{\alpha \gamma} \log ^{-\sigma}\left(2+\tau(x, y)^{-\gamma}\right)$, then

$$
H(t, x, y) \approx t^{-\frac{n}{\alpha \gamma}} \log ^{-\frac{\sigma n}{\alpha \gamma}}\left(2+t^{-1}\right)
$$

•. if $t<\tau(x, y)^{\alpha \gamma} \log ^{-\sigma}\left(2+\tau(x, y)^{-\gamma}\right)$, then

$$
H(t, x, y) \approx t \tau(x, y)^{-\alpha \gamma-n} \log ^{\sigma}\left(2+\tau(x, y)^{-\gamma}\right) .
$$

Example 5.3. Let $(\mathscr{X}, \tau)$ be a complete manifold without boundary, having nonnegative Ricci curvature. Then by [37], the heat kernel corresponding to the Laplace-Beltrami operator on $\mathscr{X}$ satisfies estimates (5.1) with

$$
\Phi_{1}(s)=e^{-C_{1} s^{2}}, \quad \Phi_{2}(s)=e^{-C_{2} s^{2}} .
$$

Now, one can take $\mathbf{T}$ with a Lévy-Khintchine exponent regularly varying at infinity with index $\alpha \in(0,1)$ and apply Claim 5.1 to obtain the asymptotic behaviour of the subordinate process.

\subsection{Green function estimates}

Let $\mathbf{T}=\left(T_{t}: t \geq 0\right)$ be a subordinator with the Laplace exponent $\phi$. If $-\phi^{\prime \prime}$ has the weak lower scaling property of index $\alpha-2$ for some $\alpha>0$, then the probability distribution of $T_{t}$ has a density $p(t, \cdot)$; see Theorem 3.3. In this section we want to derive sharp estimates on the Green function based on Sections 3 and 4. Let us recall that the Green function is

$$
G(x)=\int_{0}^{\infty} p(t, x) \mathrm{d} t, \quad x>0 .
$$

We set

$$
f(x)=\frac{\varphi(x)}{\phi^{\prime}(x)}, \quad x>0 .
$$

Let us denote by $f^{-1}$ the generalised inverse of $f$; that is,

$$
f^{-1}(x)=\sup \left\{r>0: f^{*}(r)=x\right\}
$$

where

$$
f^{*}(r)=\sup _{0<x \leq r} f(x)
$$


Notice that by (2.9) and Proposition 2.3, for all $x>x_{0}$,

$$
f^{*}(x) \lesssim x
$$

In view of (4.2) and Proposition 4.3, the function $\varphi$ is almost increasing; thus, by monotonicity of $\phi^{\prime}, f$ is almost increasing as well. Therefore, there is $c_{0} \in(0,1]$ such that for all $x>x_{0}$,

$$
c_{0} f^{*}(x) \leq f(x) \leq f^{*}(x)
$$

Moreover, $f$ has the doubling property on $\left(x_{0}, \infty\right)$. Since $\varphi$ belongs to $\operatorname{WLSC}\left(\alpha, c, x_{0}\right)$, by monotonicity of $\phi^{\prime}$, we conclude that $f$ belongs to $\operatorname{WLSC}\left(\alpha, c, x_{0}\right)$. It follows that $f^{-1} \in \mathrm{WUSC}\left(1 / \alpha, C, f^{*}\left(x_{0}\right)\right)$ for some $C \geq 1$ and since $f^{-1}$ is increasing, we infer that $f^{-1}$ also has doubling property on $\left(f^{*}\left(x_{0}\right), \infty\right)$.

Proposition 5.4. Suppose that $b=0$ and $-\phi^{\prime \prime} \in \operatorname{WLSC}\left(\alpha-2, c, x_{0}\right)$ for some $c \in(0,1]$, $x_{0} \geq 0$ and $\alpha>0$. Then for each $A>0$ and $M>0$ there is $C \geq 1$ so that for all $x<A / x_{0}$,

$$
C^{-1} \frac{1}{x \phi(1 / x)} \leq \int_{\frac{x}{\phi^{\prime}\left(f^{-1}(M / x)\right)}}^{\infty} p(t, x) \mathrm{d} t \leq C \frac{1}{x \phi(1 / x)} .
$$

In particular, for each $A>0$ there is $C>0$ such that for all $x<A / x_{0}$,

$$
G(x) \geq C \frac{1}{x \phi(1 / x)} .
$$

Proof. For $M>0$ and $x>0$ we set

$$
I_{M}(x)=\int_{\frac{x}{\phi^{\prime}\left(f^{-1}(M / x)\right)}}^{\infty} p(t, x) \mathrm{d} t .
$$

Let us first show that for each $M>0$ there are $A_{M}>0$ and $C \geq 1$ such that for all $x<A_{M} / x_{0}$,

$$
C^{-1} \frac{1}{x \phi(1 / x)} \leq \int_{\frac{x}{\phi^{\prime}\left(f^{-1}(M / x)\right)}}^{\infty} p(t, x) \mathrm{d} t \leq C \frac{1}{x \phi(1 / x)}
$$

Let

$$
A_{M}=\min \left\{M, c_{0}^{-1} M_{0}\right\} \cdot \min \left\{1, \frac{x_{0}}{f^{*}\left(x_{0}\right)}\right\}
$$

where $M_{0}$ is determined in Corollary 3.5, and $c_{0}$ is taken from (5.15). We claim that the following holds true.

Claim 5.5. For each $M>0$ there is $C \geq 1$ so that for all $x<A_{M} / x_{0}$,

$$
C^{-1} \frac{1}{\phi^{\prime}\left(f^{-1}(1 / x)\right)} \leq I_{M}(x) \leq C \frac{1}{\phi^{\prime}\left(f^{-1}(1 / x)\right)} .
$$


Suppose that

$$
t>\frac{x}{\phi^{\prime}\left(f^{-1}\left(M_{1} / x\right)\right)}
$$

with $M_{1}=c_{0}^{-1} M_{0}$. Notice that for $x<A_{M} / x_{0}$, we have $x<M_{1} / f^{*}\left(x_{0}\right)$. Hence, $x_{0} \leq$ $f^{-1}\left(M_{1} / x\right)$; thus, by monotonicity of $\phi^{\prime}$, we obtain

$$
\begin{aligned}
\frac{x}{t} & \leq \phi^{\prime}\left(f^{-1}\left(M_{1} / x\right)\right) \\
& \leq \phi^{\prime}\left(x_{0}\right) .
\end{aligned}
$$

Moreover, for $w=\left(\phi^{\prime}\right)^{-1}(x / t)$, the condition (5.18) implies that

$$
f^{*}(w) \geq M_{1} / x
$$

which together with (5.15) gives

$$
\begin{aligned}
t \varphi(w)=x f(w) & \geq c_{0} x f^{*}(w) \\
& \geq M_{0} .
\end{aligned}
$$

Now, to justify the claim, let us first consider $M \geq M_{1}$. In view of (5.19) and (5.20), we can apply Corollary 3.5 to get

$$
I_{M}(x) \approx \int_{\frac{x}{\phi^{\prime}\left(f^{-1}(M / x)\right)}}^{\infty} \frac{1}{\sqrt{t\left(-\phi^{\prime \prime}(w)\right)}} \exp \left\{-t\left(\phi(w)-w \phi^{\prime}(w)\right)\right\} \mathrm{d} t .
$$

Since by Proposition 4.1 and Remark 3.2, for all $w>x_{0}$,

$$
\begin{aligned}
\phi(w)-w \phi^{\prime}(w) & \approx h(1 / w) \\
& \approx K(1 / w) \\
& \approx w^{2}\left(-\phi^{\prime \prime}(w)\right),
\end{aligned}
$$

after the change of variables $t=x / \phi^{\prime}(s)$, we can find $C_{2} \geq 1$ such that for all $x<A_{M} / x_{0}$,

$$
\begin{aligned}
\int_{f^{-1}(M / x)}^{\infty} \exp \left\{-C_{2} x f(s)\right\} \sqrt{x f(s)} \frac{\mathrm{d} s}{s \phi^{\prime}(s)} & \lesssim I_{M}(x) \\
& \lesssim \int_{f^{-1}(M / x)}^{\infty} \exp \left\{-C_{2}^{-1} x f(s)\right\} \sqrt{x f(s)} \frac{\mathrm{d} s}{s \phi^{\prime}(s)}
\end{aligned}
$$

Recall that $f^{-1}$ has the doubling property on $\left(f^{*}\left(x_{0}\right), \infty\right)$. Using now Proposition 2.3 and (5.15), we get

$$
\begin{aligned}
I_{M}(x) & \gtrsim \int_{f^{-1}(M / x)}^{2 f^{-1}(M / x)} \exp \left\{-C_{2} x f^{*}(s)\right\} \sqrt{x f^{*}(s)} \frac{\mathrm{d} s}{\phi(s)} \\
& \gtrsim \frac{1}{\phi\left(f^{-1}(M / x)\right)} f^{-1}(M / x) \\
& \gtrsim \frac{1}{\phi^{\prime}\left(f^{-1}(M / x)\right)},
\end{aligned}
$$


where the implicit constants may depend on $M$. Therefore, by monotonicity of $f^{-1}$ and $\phi^{\prime}$, the estimate (5.22) gives

$$
I_{M}(x) \gtrsim \frac{1}{\phi^{\prime}\left(f^{-1}(1 / x)\right)} .
$$

This proves the first inequality in (5.17).

We next observe that (5.14) entails that $f^{-1}(s) \gtrsim s$ for $s>f^{*}\left(x_{0}\right)$; thus, by (5.23),

$$
\begin{aligned}
G(x) \geq I_{M_{1}}(x) & \gtrsim \frac{1}{\phi^{\prime}(1 / x)} \\
& \gtrsim \frac{1}{x \phi(1 / x)}
\end{aligned}
$$

where the last estimate follows by Proposition 2.3.

We next show the second inequality in (5.17). By (5.21), Proposition 2.3 and monotonicity of $\phi$,

$$
\begin{aligned}
I_{M}(x) & \lesssim \int_{f^{-1}(M / x)}^{\infty} \exp \left\{-C_{2}^{-1} x f(s)\right\} \sqrt{x f(s)} \frac{\mathrm{d} s}{\phi(s)} \\
& \leq \frac{1}{\phi\left(f^{-1}(M / x)\right)} \int_{f^{-1}(M / x)}^{\infty} \exp \left\{-C_{2}^{-1} x f(s)\right\} \sqrt{x f(s)} \mathrm{d} s \\
& \leq \frac{1}{\phi\left(f^{-1}(M / x)\right)} \int_{f^{-1}(M / x)}^{\infty} \exp \left\{-\frac{1}{2 C_{2}} x f(s)\right\} \mathrm{d} s
\end{aligned}
$$

where in the last inequality we have used

$$
\exp \left\{-C_{2}^{-1} x f(s)\right\} \sqrt{x f(s)} \leq \exp \left\{-\frac{1}{2 C_{2}} x f(s)\right\} .
$$

Since $\varphi \in \operatorname{WLSC}\left(\alpha, c, x_{0}\right)$, by [6, Lemma 16],

$$
\int_{\mathbb{R}} \exp \left\{-C_{2}^{-1} x f(|s|)\right\} \mathrm{d} s \lesssim f^{-1}\left(M_{1} / x\right) .
$$

Finally, the doubling property of $f^{-1}$, monotonicity of $\phi$ and Proposition 2.3 give

$$
\begin{aligned}
I_{M}(x) & \lesssim \frac{1}{\phi\left(f^{-1}(M / x)\right)} f^{-1}\left(M_{1} / x\right) \\
& \lesssim \frac{1}{\phi^{\prime}\left(f^{-1}(1 / x)\right)}
\end{aligned}
$$

where the implied constant may depend on $M$. This finishes the proof of (5.17) for $M \geq$ $M_{1}$.

We next consider $0<M<M_{1}$. By monotonicity, the lower estimate remains valid for all $M>0$. Therefore, it is enough to show that for each $0<M<M_{1}$, there is $C \geq 1$ such that for all $x<A_{M} / x_{0}$,

$$
\int_{\frac{x}{\phi^{\prime}\left(f^{-1}(M / x)\right)}}^{\frac{x}{\left.\phi^{-1}\left(M_{1} / x\right)\right)}} p(t, x) \mathrm{d} t \leq C \frac{1}{\phi^{\prime}\left(f^{-1}(1 / x)\right)} .
$$


By [23, Theorem 3.1], there is $t_{0}>0$ such that for all $0<t<t_{0}$,

$$
p(t, x) \lesssim \varphi^{-1}(1 / t)
$$

If $x_{0}=0$, then $t_{0}=\infty$. Since $\varphi$ is almost increasing we have

$$
\begin{aligned}
\frac{x}{\phi^{\prime}\left(f^{-1}\left(M_{1} / x\right)\right)} & \leq \frac{M_{1}}{\varphi\left(f^{-1}\left(M_{1} / x\right)\right)} \\
& \lesssim \frac{M_{1}}{\varphi\left(f^{-1}\left(M_{1} x_{0} / A\right)\right)} .
\end{aligned}
$$

Hence, by continuity and positivity of $p(t, x)$ and $\varphi^{-1}(1 / t)$, we can take

$$
t_{0} \geq \frac{x}{\phi^{\prime}\left(f^{-1}\left(M_{1} / x\right)\right)}
$$

Therefore, by the change of variables $t=x / \phi^{\prime}(s)$ we get

$$
\begin{aligned}
\int_{\frac{x}{\phi^{\prime}\left(f^{-1}(M / x)\right)}}^{\frac{x}{\phi^{\prime}\left(f^{-1}\left(M_{1} / x\right)\right)}} p(t, x) \mathrm{d} t & \lesssim \int_{\frac{x}{\phi^{\prime}\left(f^{-1}(M / x)\right)}}^{\frac{x}{\phi^{\prime}\left(f^{-1}\left(M_{1} / x\right)\right)}} \varphi^{-1}(1 / t) \mathrm{d} t \\
& =x \int_{f^{-1}(M / x)}^{f^{-1}\left(M_{1} / x\right)} \varphi^{-1}\left(\frac{\phi^{\prime}(s)}{x}\right) f(s) \frac{\mathrm{d} s}{s^{2} \phi^{\prime}(s)} .
\end{aligned}
$$

Next, by monotonicity and the doubling property of $f^{-1}$ and $\phi^{\prime}$, we obtain

$$
\begin{aligned}
\int_{\frac{x}{\phi^{\prime}\left(f^{-1}(M / x)\right)}}^{\frac{x}{\left.\phi^{\prime}\left(M_{1} / x\right)\right)}} p(t, x) \mathrm{d} t & \lesssim \frac{1}{\left(f^{-1}(M / x)\right)^{2}} \cdot \frac{1}{\phi^{\prime}\left(f^{-1}\left(M_{1} / x\right)\right)} \int_{f^{-1}(M / x)}^{f^{-1}\left(M_{1} / x\right)} \varphi^{-1}\left(\frac{\phi^{\prime}(s)}{x}\right) \mathrm{d} s \\
& \lesssim \frac{1}{\left(f^{-1}(1 / x)\right)^{2}} \cdot \frac{1}{\phi^{\prime}\left(f^{-1}(1 / x)\right)} \int_{f^{-1}(M / x)}^{f^{-1}\left(M_{1} / x\right)} \varphi^{-1}\left(\frac{\phi^{\prime}(s)}{x}\right) \mathrm{d} s
\end{aligned}
$$

Since by $(5.15)$ for $s \geq f^{-1}(M / x)$ we have

$$
\frac{\phi^{\prime}(s)}{x}=\frac{\varphi(s)}{x f(s)} \lesssim \varphi^{*}(s),
$$

by monotonicity of $\varphi^{-1}$, Proposition 4.3 , Remark 4.4 and the doubling property of $f^{-1}$ and $\varphi^{-1}$, we get

$$
\int_{f^{-1}(M / x)}^{f^{-1}\left(M_{1} / x\right)} \varphi^{-1}\left(\frac{\phi^{\prime}(s)}{x}\right) \mathrm{d} s \lesssim\left(f^{-1}(1 / x)\right)^{2},
$$

which together with (5.25) gives (5.17) for $0<M<M_{1}$. This completes the proof of Claim 5.5.

Our next task is to deduce (5.16) from Claim 5.5. By Lemma 2.9 and Proposition 2.3, there is a complete Bernstein function $\tilde{\phi}$ such that $\tilde{\phi} \approx \phi$ and

$$
f(x) \approx \tilde{f}(x)=\frac{x^{2}\left(-\tilde{\phi}^{\prime \prime}(x)\right)}{\tilde{\phi}^{\prime}(x)}
$$


for all $x>x_{0}$. Let $\tilde{\mathbf{T}}$ be a subordinator with the Laplace exponent $\tilde{\phi}$. By $\tilde{p}(t, \cdot)$ we denote the density of the probability distribution of $\tilde{T}_{t}$. We set

$$
\tilde{G}(x)=\int_{0}^{\infty} \tilde{p}(t, x) \mathrm{d} t
$$

and

$$
\tilde{I}_{M}(x)=\int_{\frac{x}{\tilde{\phi}^{\prime}\left(\tilde{f}^{-1}(M / x)\right)}}^{\infty} \tilde{p}(t, x) \mathrm{d} t .
$$

Fix $M>0$. By Claim 5.5, there is $A_{M}>0$ such that for all $x<A_{M} / x_{0}$,

$$
\begin{aligned}
\tilde{I}_{M}(x) & \approx \frac{1}{\tilde{\phi}^{\prime}\left(\tilde{f}^{-1}(1 / x)\right)} \\
& \approx \frac{1}{\phi^{\prime}\left(f^{-1}(1 / x)\right)} .
\end{aligned}
$$

On the other hand, since $\tilde{\phi}$ is the complete Bernstein function, by (5.24) and [34, Corollary 2.6], there is $C_{3} \geq 1$ such that

$$
C_{3}^{-1} \frac{1}{x \tilde{\phi}(1 / x)} \leq \tilde{I}_{M}(x) \leq \tilde{G}(x) \leq C_{3} \frac{1}{x \tilde{\phi}(1 / x)} .
$$

Therefore, by (5.26), for $x<A_{M} / x_{0}$,

$$
\begin{aligned}
\frac{1}{\phi^{\prime}\left(f^{-1}(1 / x)\right)} \approx \tilde{I}_{M}(x) & \approx \frac{1}{x \tilde{\phi}(1 / x)} \\
& \approx \frac{1}{x \phi(1 / x)}
\end{aligned}
$$

and (5.16) follows for all $A \leq A_{M}$. Let us now consider $A>A_{M}$. Observe that the functions

$$
\left[\frac{A_{M}}{x_{0}}, \frac{A}{x_{0}}\right] \ni x \mapsto \frac{1}{x \phi(1 / x)}
$$

and

$$
\left[\frac{A_{M}}{x_{0}}, \frac{A}{x_{0}}\right] \ni x \mapsto \int_{\frac{x}{\phi^{\prime}\left(f^{-1}(M / x)\right)}}^{\infty} p(t, x) \mathrm{d} t
$$

are both positive and continuous; thus, they are bounded for each $A$. Therefore, at the possible expense of worsening the constant, we can conclude the proof of the proposition.

Proposition 5.6. Suppose that $b=0,-\phi^{\prime \prime} \in \operatorname{WLSC}\left(\alpha-2, c, x_{0}\right)$ for some $c \in(0,1], x_{0} \geq 0$ and $\alpha>0$ and that the Lévy measure $\nu(\mathrm{d} x)$ is absolutely continuous with respect to the Lebesgue measure with a monotone density $\nu(x)$. Then there is $\epsilon \in(0,1)$ such that for each $A>0$, there is $C \geq 1$ such that for all $x<A / x_{0}$,

$$
\int_{0}^{\frac{x}{\phi^{\prime}\left(f^{-1}(1 / x)\right)} \epsilon} p(t, x) \mathrm{d} t \leq C \frac{1}{x \phi(1 / x)} .
$$


Proof. In view of (5.27), it is enough to show that for some $\epsilon \in(0,1)$ and all $A>0$ there is $C \geq 1$, such that for all $x<A / x_{0}$,

$$
\int_{0}^{\frac{x}{\phi^{\prime}\left(f^{-1}(1 / x)\right)} \epsilon} p(t, x) \mathrm{d} t \leq C \frac{1}{\phi^{\prime}\left(f^{-1}(1 / x)\right)} .
$$

Let $\epsilon \in(0,1)$ and

$$
A=\min \left\{1, \frac{x_{0}}{f^{*}\left(x_{0}\right)}\right\}
$$

Suppose that

$$
t \leq \frac{x}{\phi^{\prime}\left(f^{-1}(1 / x)\right)} \epsilon
$$

that is,

$$
t \leq \frac{1}{\varphi^{*}\left(f^{-1}(1 / x)\right)} \epsilon .
$$

Hence, by monotonicity of $\varphi^{-1}$ and $\phi^{\prime}$,

$$
\begin{aligned}
x \geq \frac{1}{f^{*}\left(\varphi^{-1}(\epsilon / t)\right)} & =\frac{t}{\epsilon} \phi^{\prime}\left(\varphi^{-1}(\epsilon / t)\right) \\
& \geq \frac{t}{\epsilon} \phi^{\prime}\left(\varphi^{-1}(1 / t)\right) .
\end{aligned}
$$

By Proposition 4.3 and the scaling property of $\phi^{\prime}$, there are $c \in(0,1]$ and $C \geq 1$ such that

$$
\begin{aligned}
x & \geq \frac{t}{\epsilon} \phi^{\prime}\left(C \psi^{-1}(1 / t)\right) \\
& \geq \frac{t}{\epsilon} c C^{\alpha-1} \phi^{\prime}\left(\psi^{-1}(1 / t)\right) .
\end{aligned}
$$

Therefore, by taking $\epsilon=(2 e)^{-1} c C^{\alpha-1}$, we get

$$
x \geq 2 e t \phi^{\prime}\left(\psi^{-1}(1 / t)\right) .
$$

Since $\nu(x)$ is the monotone density of $\nu(\mathrm{d} x)$, by Theorem 4.8 we get

$$
\int_{0}^{\frac{x}{\phi^{\prime}\left(f^{-1}(1 / x)\right)} \epsilon} p(t, x) \mathrm{d} t \lesssim \frac{\varphi(1 / x)}{x}\left(\frac{x}{\phi^{\prime}\left(f^{-1}(1 / x)\right)}\right)^{2} .
$$

By (5.14), $f^{-1}(s) \gtrsim s$ for $s>f^{*}\left(x_{0}\right)$; thus, using (4.4),

$$
\frac{x \varphi(1 / x)}{\phi^{\prime}\left(f^{-1}(1 / x)\right)} \leq \frac{\varphi(1 / x)}{\varphi\left(f^{-1}(1 / x)\right)} \lesssim 1,
$$

which entails (5.28). The extension to arbitrary $A$ follows by continuity and positivity argument as in the proof of Proposition 5.4.

It is possible to get the same conclusion as in Proposition 5.6 without imposing the existence of the monotone density of $\nu(\mathrm{d} x)$; however, instead we need to assume the weak upper scaling property in $-\phi^{\prime \prime}$. 
Proposition 5.7. Suppose that $b=0$ and $-\phi^{\prime \prime} \in \operatorname{WLSC}\left(\alpha-2, c, x_{0}\right) \cap \operatorname{WUSC}\left(\beta-2, C, x_{0}\right)$ for some $c \in(0,1], C \geq 1, x_{0} \geq 0$ and $\frac{1}{2}<\alpha \leq \beta<1$. Then there is $\epsilon \in(0,1)$ such that for each $A>0$, there is $C_{1} \geq 1$, so that for all $x<A / x_{0}$,

$$
\int_{0}^{\frac{x}{\phi^{\prime}\left(f^{-1}(1 / x)\right)} \epsilon} p(t, x) \mathrm{d} t \leq C_{1} \frac{1}{x \phi(1 / x)} .
$$

Proof. Let

$$
A=\min \left\{1, \frac{x_{0}}{f^{*}\left(x_{0}\right)}\right\}
$$

By repeating the same reasoning as in the proof of Proposition 5.6, we can see that the condition

$$
t \leq \frac{x}{\phi^{\prime}\left(f^{-1}(1 / x)\right)} \epsilon
$$

implies

$$
x \geq 2 e t \phi^{\prime}\left(\psi^{-1}(1 / t)\right)
$$

for $\epsilon=(2 e)^{-1} c C^{\alpha-1}$. Therefore, we can apply Theorem 4.7 to get

$$
\int_{0}^{\frac{x}{\phi^{\prime}\left(f^{-1}(1 / x)\right)} \epsilon} p(t, x) \mathrm{d} t \lesssim \varphi(1 / x) \int_{0}^{\frac{x}{\phi^{\prime}\left(f^{-1}(1 / x)\right)} \epsilon} t \varphi^{-1}(1 / t) \mathrm{d} t,
$$

where the implied constant may depend on $\epsilon$. Since $\alpha>\frac{1}{2}$, by Proposition 4.3, [1, Theorem $3]$ and the doubling property of $\varphi^{-1}$, we obtain

$$
\begin{aligned}
\int_{0}^{\frac{x}{\phi^{\prime}\left(f^{-1}(1 / x)\right)} \epsilon} t \varphi^{-1}(1 / t) \mathrm{d} t & \lesssim\left(\frac{x}{\phi^{\prime}\left(f^{-1}(1 / x)\right)}\right)^{2} \varphi^{-1}\left(\frac{\phi^{\prime}\left(f^{-1}(1 / x)\right)}{\epsilon x}\right) \\
& \lesssim\left(\frac{x}{\phi^{\prime}\left(f^{-1}(1 / x)\right)}\right)^{2} \varphi^{-1}\left(\frac{\phi^{\prime}\left(f^{-1}(1 / x)\right)}{x}\right) .
\end{aligned}
$$

In view of (5.15), we have

$$
\begin{aligned}
\frac{\phi^{\prime}\left(f^{-1}(1 / x)\right)}{x} & =\frac{\varphi^{*}\left(f^{-1}(1 / x)\right)}{x f^{*}\left(f^{-1}(1 / x)\right)} \\
& \lesssim \varphi^{*}\left(f^{-1}(1 / x)\right)
\end{aligned}
$$

thus, by Proposition 4.3 and Remark 4.4,

$$
\begin{aligned}
\frac{\varphi(1 / x) x^{2}}{\phi^{\prime}\left(f^{-1}(1 / x)\right)} \varphi^{-1}\left(\frac{\phi^{\prime}\left(f^{-1}(1 / x)\right)}{x}\right) & \lesssim \frac{\varphi^{*}(1 / x) x^{2}}{\phi^{\prime}\left(f^{-1}(1 / x)\right)} f^{-1}(1 / x) \\
& =x f^{-1}(1 / x) \frac{\varphi^{*}(1 / x)}{\varphi^{*}\left(f^{-1}(1 / x)\right)}
\end{aligned}
$$


In view of Propositions 2.3 and 4.6, we have $f(s) \approx s$ for $s>x_{0}$; thus, $f^{-1}(s) \approx s$, for $s>f^{*}\left(x_{0}\right)$. Hence,

$$
\frac{\varphi(1 / x) x^{2}}{\phi^{\prime}\left(f^{-1}(1 / x)\right)} \varphi^{-1}\left(\frac{\phi^{\prime}\left(f^{-1}(1 / x)\right)}{x}\right) \lesssim 1 .
$$

Therefore, by (5.31) and (5.32), we conclude that

$$
\int_{0}^{\frac{x}{\phi^{\prime}\left(f^{-1}(1 / x)\right)} \epsilon} p(t, x) \mathrm{d} t \lesssim \frac{1}{\phi^{\prime}\left(f^{-1}(1 / x)\right)},
$$

which, by Proposition 5.4 and (5.27), entails (5.30). The extension to arbitrary $A$ follows by positivity and continuity argument.

Theorem 5.8. Let $\mathbf{T}$ be a subordinator with the Laplace exponent $\phi$. Suppose that

$$
\phi \in W L S C\left(\alpha, c, x_{0}\right) \cap W U S C\left(\beta, C, x_{0}\right)
$$

for some $c \in(0,1], C \geq 1, x_{0} \geq 0$ and $0<\alpha \leq \beta<1$. We assume that one of the following conditions holds:

(i) The Lévy measure $\nu(\mathrm{d} x)$ is absolutely continuous with respect to the Lebesgue measure with monotone density $\nu(x)$, or

(ii) $\alpha>\frac{1}{2}$.

Then for each $A>0$ there is $C_{1} \geq 1$ such that for all $x<A / x_{0}$,

$$
C_{1}^{-1} \frac{1}{x \phi(1 / x)} \leq G(x) \leq C_{1} \frac{1}{x \phi(1 / x)} .
$$

Proof. By Corollary $2.7,-\phi^{\prime \prime} \in \mathrm{WLSC}\left(\alpha-2, c, x_{0}\right) \cap \operatorname{WUSC}\left(\beta-2, C, x_{0}\right)$. Let $p(t, \cdot)$ be the transition density of $T_{t}$. In view of Propositions 5.4, 5.6 and 5.7 and (5.27), it is enough to show that for each $A>0$ and $\epsilon \in(0,1)$ there is $C_{1}>0$ such that for all $x<A / x_{0}$,

$$
\int_{\frac{x}{\phi^{\prime}\left(f^{-1}(1 / x)\right)} \epsilon}^{\frac{x}{\left.\phi^{-1}(1 / x)\right)}} p(t, x) \mathrm{d} t \leq C_{1} \frac{1}{\phi^{\prime}\left(f^{-1}(1 / x)\right)} .
$$

By [23, Theorem 3.1], there is $t_{0}>0$ such that for all $t \in\left(0, t_{0}\right)$,

$$
p(t, x) \lesssim \varphi^{-1}(1 / t) .
$$

If $x_{0}=0$, then $t_{0}=\infty$. We can take

$$
t_{0} \geq \frac{x}{\phi^{\prime}\left(f^{-1}(1 / x)\right)} .
$$

Therefore, by monotonicity of $\varphi^{-1}$, we get

$$
\begin{aligned}
\int_{\frac{x}{\phi^{\prime}\left(f^{-1}(1 / x)\right)} \epsilon}^{\frac{x}{\phi^{\prime}\left(f^{-1}(1 / x)\right)}} p(t, x) \mathrm{d} t & \lesssim \int_{\frac{x}{\phi^{\prime}\left(f^{-1}(1 / x)\right)} \epsilon}^{\frac{x}{\phi^{\prime}\left(f^{-1}(1 / x)\right)}} \varphi^{-1}(1 / t) \mathrm{d} t \\
& \leq \frac{x}{\phi^{\prime}\left(f^{-1}(1 / x)\right)} \varphi^{-1}\left(\frac{\phi^{\prime}\left(f^{-1}(1 / x)\right)}{\epsilon x}\right) .
\end{aligned}
$$


By the doubling property of $\varphi^{-1}$, definition of $f$ and Remark 4.4,

$$
\begin{aligned}
\varphi^{-1}\left(\frac{\phi^{\prime}\left(f^{-1}(1 / x)\right)}{\epsilon x}\right) & \lesssim \varphi^{-1}\left(\frac{\phi^{\prime}\left(f^{-1}(1 / x)\right)}{x}\right) \\
& \leq f^{-1}(1 / x) \\
& \lesssim \frac{1}{x}
\end{aligned}
$$

since by the weak upper scaling property of $-\phi^{\prime \prime}, f(s) \approx s$ for all $s>f^{*}\left(x_{0}\right)$. Consequently, we obtain (5.33) and the theorem follows.

Acknowledgements. We thank Professor Jerzy Zabczyk for drawing our attention to the problem considered in this article. The main results of this article were presented at the XV Probability Conference held from 21 to 25 May 2018 in Będlewo, Poland, and at the Semigroups of Operators: Theory and Applications Conference held from 30 September to 5 October 2018 in Kazimierz Dolny, Poland. We thank the organisers for the invitations. The authors were partially supported by the National Science Centre (Poland) (Grant No. 2016/23/B/ST1/01665).

Competing Interest. None.

\section{References}

[1] S. Aljančić and D. AranĐelović, O-regularly varying functions, Publ. Inst. Math. (Beograd) (N.S.) 22(36): (1977), 5-22.

[2] M. T. BARLow, Diffusions on fractals, in Lectures on Probability Theory and Statistics (Saint-Flour, 1995), Vol. 1690 of Lecture Notes in Math. (Springer, Berlin, 1998), 1-121.

[3] N. H. Bingham, C. M. Goldie and J. L. Teugels, Regular Variation, Vol. 27 of Encyclopedia of Mathematics and Its Applications (Cambridge University Press, Cambridge 1989).

[4] R. M. Blumenthal and R. K. Getoon, Some theorems on stable processes, Trans. Amer. Math. Soc. 95 (1960), 263-273.

[5] S. Bochner, Diffusion equation and stochastic processes, Proc. Nat. Acad. Sci. U.S.A. 35, (1949). 368-370.

[6] K. Bogdan, T. Grzywny and M. Ryznar, Density and tails of unimodal convolution semigroups, J. Funct. Anal. 266(6) (2014), 3543-3571.

[7] K. Bogdan, P. Sztonyk, and V. Knopova, Heat kernel of anisotropic nonlocal operators, Doc. Math. 25 (2020), 1-54.

[8] J. Burridge, A. Kuznetsov, M. Kwaśnicki and A. E. Kyprianou, New families of subordinators with explicit transition probability semigroup, Stochastic Process. Appl. 124(10) (2014), 3480-3495.

[9] Z.-Q. Chen, Time fractional equations and probabilistic representation, Chaos Solitons Fractals 102 (2017), 168-174.

[10] Z.-Q. Chen, P. Kim and T. Kumagai, Weighted Poincaré inequality and heat kernel estimates for finite range jump processes, Math. Ann. 342(4) (2008), 833-883.

[11] Z.-Q. Chen, P. Kim and T. Kumagai, Global heat kernel estimates for symmetric jump processes, Trans. Amer. Math. Soc. 363(9) (2011), 5021-5055. 
[12] Z.-Q. Chen, P. Kim, T. Kumagai And J. Wang, Heat kernel estimates for time fractional equations, Forum Math. 30(5) (2018), 1163-1192.

[13] Z.-Q. Chen, P. Kim, T. Kumagai and J. Wang, Time fractional Poisson equations: representations and estimates, J. Funct. Anal. 278(2) (2020), 108311, 48.

[14] Z.-Q. Chen And T. Kumagai, Heat kernel estimates for jump processes of mixed types on metric measure spaces, Probab. Theory Related Fields 140(1-2) (2008), 277-317.

[15] W. Cygan, T. Grzywny and B. Trojan, Asymptotic behavior of densities of unimodal convolution semigroups, Trans. Amer. Math. Soc. 369(8) (2017), 5623-5644.

[16] M. A. Fahrenwaldt, Heat kernel asymptotics of the subordinator and subordinate Brownian motion, J. Evol. Equ. 19(1) (2019), 33-70.

[17] B. E. Fristedt And W. E. Pruitt, Uniform lower functions for subordinators, $Z$. Wahrscheinlichkeitstheorie und Verw. Gebiete 24 (1972), 63-70.

[18] A. Gomilko And Y. Tomilov, On subordination of holomorphic semigroups, Adv. Math. 283 (2015), 155-194.

[19] L. M. Graves, The Theory of Functions of Real Variables, 1st ed. (McGraw-Hill Book Company, New York, 1946).

[20] T. Grzywny, On Harnack inequality and Hölder regularity for isotropic unimodal Lévy processes, Potential Anal. 41(1) (2014), 1-29.

[21] T. Grzywny, M. Ryznar and B. Trojan, Asymptotic behaviour and estimates of slowly varying convolution semigroups, Int. Math. Res. Not. IMRN 2019(23) (2019), 7193-7258.

[22] T. Grzywny And K. Szczypkowski, Estimates of heat kernels of non-symmetric Lévy processes, Forum Mathematicum 33(5) (2021), 1207-1236.

[23] T. Grzywny And K. Szczypkowski, Lévy processes: concentration function and heat kernel bounds, Bernoulli 26(4) (2020), 3191-3223.

[24] J. Hawkes, A lower Lipschitz condition for the stable subordinator, Z. Wahrscheinlichkeitstheorie und Verw. Gebiete 17 (1971), 23-32.

[25] S. Hiraba, Asymptotic behaviour of densities of multi-dimensional stable distributions, Tsukuba J. Math. 18(1) (1994), 223-246.

[26] A. Iksanov, Z. Kabluchko, A. Marynych and G. Shevchenko, Fractionally integrated inverse stable subordinators. Stochastic Process, Appl., 127(1) (2017), 80-106.

[27] Y. Ishikawa, Asymptotic behavior of the transition density for jump type processes in small time, Tohoku Math. J. (2) 46(4) (1994), 443-456.

[28] Y. Ishikawa, Density estimate in small time for jump processes with singular Lévy measures, Tohoku Math. J. (2) 53(2) (2001), 183-202.

[29] N. Jacob, Pseudo Differential Operators and Markov Processes, Vol. I (Imperial College Press, London, 2001).

[30] N. C. JAin And W. E. Pruitt, Lower tail probability estimates for subordinators and nondecreasing random walks, Ann. Probab. 15(1) (1987), 75-101.

[31] K. Kaleta and P. Sztonyk, Upper estimates of transition densities for stable-dominated semigroups, J. Evol. Equ. 13(3) (2013), 633-650.

[32] K. Kaleta and P. Sztonyk, Estimates of transition densities and their derivatives for jump Lévy processes, J. Math. Anal. Appl. 431(1) (2015), 260-282.

[33] P. Kim And A. Mimica, Estimates of Dirichlet heat kernels for subordinate Brownian motions, Electron. J. Probab. 23 (2018), Paper No. 64, 45.

[34] P. Kim, R. Song ANd Z. VondraČEK, Potential theory of subordinate Brownian motions revisited, in Stochastic Analysis and Applications to Finance, Vol. 13 of Interdiscip. Math. Sci. (World Sci. Publ., Hackensack, NJ, (2012), 243-290.

[35] V. Knopova, Compound kernel estimates for the transition probability density of a Lévy process in $\mathbb{R}^{n}$, Teor. Imovirr. Mat. Stat. 89 (2013), 51-63. 
[36] V. Knopova And A. KuliK, Intrinsic small time estimates for distribution densities of Lévy processes, Random Oper. Stoch. Equ. 21(4) (2013), 321-344.

[37] P. Li And S.-T. YAU, On the parabolic kernel of the Schrödinger operator, Acta Math. 156(3-4) (1986), 153-201.

[38] M. M. Meerschaert And H.-P. Scheffler, Limit theorems for continuous-time random walks with infinite mean waiting times, J. Appl. Probab. 41(3) (2004), 623-638.

[39] A. Mimica, Heat kernel upper estimates for symmetric jump processes with small jumps of high intensity, Potential Anal. 36(2) (2012), 203-222.

[40] A. Mimica, Heat kernel estimates for subordinate Brownian motions, Proc. Lond. Math. Soc. (3) 113(5) (2016), 627-648.

[41] R. S. Phillips, On the generation of semigroups of linear operators, Pacific J. Math. 2: (1952), 343-369.

[42] J. PiCARD, Density in small time for Levy processes, ESAIM Probab. Statist. 1 (1995/97), 357-389.

[43] J. PicARD, Density in small time at accessible points for jump processes, Stochastic Process. Appl. 67(2) (1997), 251-279.

[44] G. PÓLYA, On the zeros of an integral function represented by Fourier transform, Messenger of Math. 52 (1923), 185-188.

[45] W. E. Pruitt, The growth of random walks and Lévy processes, Ann. Probab. 9(6) (1981), 948-956.

[46] W. E. Pruitt and S. J. Taylor, The potential kernel and hitting probabilities for the general stable process in $R^{N}$, Trans. Amer. Math. Soc. 146 (1969), 299-321.

[47] K.-I. Sato, Lévy Processes and Infinitely Divisible Distributions, Vol. 68 of Cambridge Studies in Advanced Mathematics (Cambridge University Press, Cambridge, 1999). Translated from the 1990 Japanese original, Revised by the author.

[48] R. L. Schilling, R. Song And Z. VondraČeK, Bernstein Functions: Theory and Applications, 2nd ed., Vol. 37 of De Gruyter Studies in Mathematics (Walter de Gruyter \& Co., Berlin, 2012).

[49] R. Song, Sharp bounds on the density, Green function and jumping function of subordinate killed BM, Probab. Theory Related Fields 128(4) (2004), 606-628.

[50] P. Sztonyk, Transition density estimates for jump Lévy processes, Stochastic Process. Appl. 121(6) (2011), 1245-1265.

[51] P. Sztonyk, Estimates of densities for Lévy processes with lower intensity of large jumps, Math. Nachr. 290(1) (2017), 120-141.

[52] R. Vasudeva And G. Divanji, Law of the iterated logarithm for the increments of stable subordinators, Stochastic Process. Appl. 28(2) (1988), 293-300.

[53] T. Watanabe, Asymptotic estimates of multi-dimensional stable densities and their applications, Trans. Amer. Math. Soc. 359(6) (2007), 2851-2879. 